THE UNIVERSITY OF MANITOBA

ELECTRICAL AND COMPUTER ENGINEERING DEPARTMENT

\title{
SYNTHESIS OF LINE SOURCE FEEDS \\ FOR CONICAL REFLECTORS AND \\ THEIR PERFORMANCE USING DIPOLE ARRAYS
}

\author{
A Dissertation \\ Presented to the Faculty of Graduate Studies \\ University of Manitoba
}

\begin{abstract}
In Partial Fulfillment
of the Requirements for the Degree of

Doctor of Philosophy

in

Electrical Engineering
\end{abstract}

by

DESSOUKI AHMED MORSY EID

JULY 1992 
National Library

of Canada

Acquisitions and

Bibliographic Services Branch

395 Wellington Street

Ottawa, Ontario
Bibliothèque nationale

du Canada

Direction des acquisitions et

des services bibliographiques

395, rue Wellington

Ottawa (Ontario)
Your lile Volre rétérence

Our fite Notre référence
The author has granted an irrevocable non-exclusive licence allowing the National Library of Canada to reproduce, Ioan, distribute or sell copies of his/her thesis by any means and in any form or format, making this thesis available to interested persons.
L'auteur a accordé une licence irrévocable et non exclusive permettant à la Bibliothèque nationale du Canada de reproduire, prêter, distribuer ou vendre des copies de sa thèse de quelque manière et sous quelque forme que ce soit pour mettre des exemplaires de cette thèse à la disposition des personnes intéressées.
The author retains ownership of the copyright in his/her thesis. Neither the thesis nor substantial extracts from it may be printed or otherwise reproduced without his/her permission.
L'auteur conserve la propriété du droit d'auteur qui protège sa thèse. Ni la thèse ni des extraits substantiels de celle-ci ne doivent être imprimés ou autrement reproduits sans son autorisation. 
SYNTHESIS OF LINE SOURCE FEEDS

FOR CONICAL REFLECTORS AND

THEIR PERFORMANCE USING DIPOLE ARRAYS

BY

DESSOURI AHMED MORSY EID

A Thesis submitted to the Faculty of Graduate Stadies of the University of Manitoba in partial fulfillment of the requirements for the degree of

DOCTOR OF PHILOSOPHY

두 1992

Permission has been granted to the LIBRARY OF THE UNTVERSITY OF MANITOBA to lend or sell copies of this thesis, to the NAIIONAL LIBRARY OF CANADA to microfilm this thesis and to lend or sell copies of the film, and UNTVERSTYY MACROFILMS to publish an abstract of this thesis.

The author reserves other publication rights, and neither the thesis nor extensive extracts from it may be printed or otherwise reproduced without the author's permission. 
I hereby declare that I am the sole author of this dissertation.

I authorize the University of Manitoba to lend this dissertation to other institutions or individuals for the purpose of scholarly research.

Dessouki Ahmed Morsy Eid

I further authorize the University of Manitoba to reproduce this dissertation by photocopying or by other means, in total or in part, at the request of other institutions or individuals for the purpose of scholarly research.

Dessouki Ahmed Morsy Eid 


\begin{abstract}
The matched feed concept is used to determine the electrical characteristics of a line source feed for conical reflector antennas. A linearly polarized plane wave incident on the reflector is used to determine the field distribution along and in the vicinity of the focal axis of the reflector. The co-polar, cross-polar and axial field components are determined and their dependence on the reflector size, the cone angle and the radial distance is studied. The results indicate that for a line source of finite diameter, its surface field distribution must contain other field components to generate a linearly polarized far field. Based on the matched feed concept, line sources in the form of simple dipole arrays are proposed and the conical reflector radiation patterns are computed. The dependence of the pattern characteristics, such as the polarization, efficiency and side lobe levels, on the dipole excitations is studied and discussed.

For the wide angle cone case, a microstrip comb line array feed is proposed and studied. A compact design is also presented. In the design an optimization technique is used to obtain the physical dimensions on one side of the substrate for the traveling wave array. Correction formulas are used to compensate for the effect of discontinuities in the structure and further correction is introduced to provide a proper impedance match by the use of an admittance transformation.
\end{abstract}




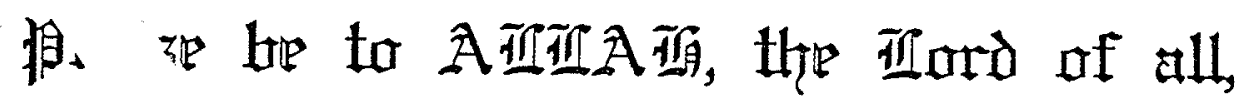
at Hles. i pratige liked by and arreptatile

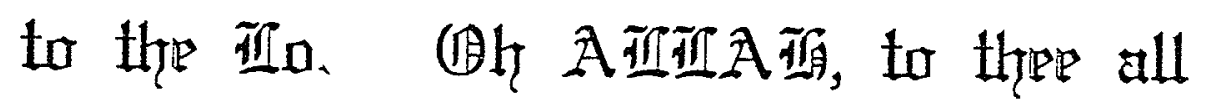
thanthfullotess. Thidour ante urith that knoulpige ant "gonm urhirh will berteftit me and ha - to trentefit

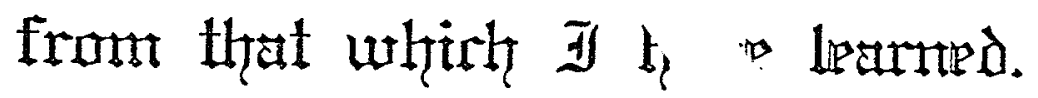




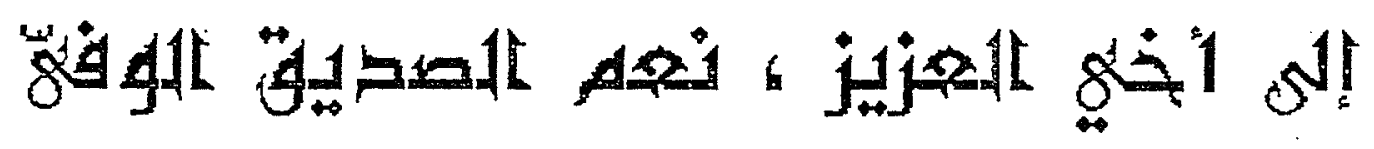

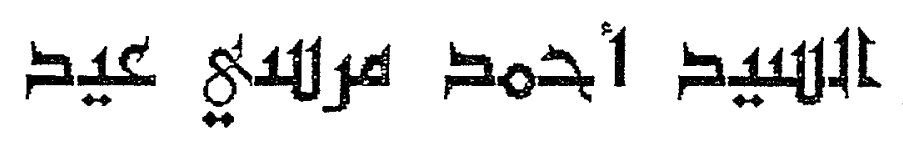

đor my dear trotther, the real frimito

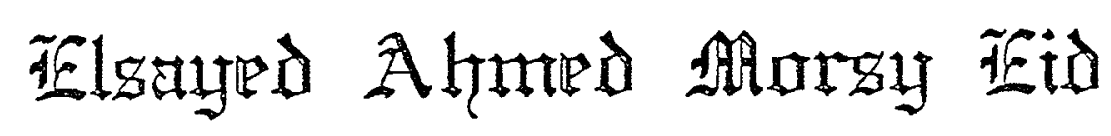




\section{ACKNOWLEDGEMENTS}

I wish to express my heart felt gratitude to Dr. L. Shafai for both his academic and personal assistance, advice and encouragement throughout the course of this investigation. I am also indebted to him for taking the time to read and evaluate my thesis through his invaluable suggestions.

Special thanks are due to Professor E. Bridges and Dr. J.J. Williams, the members of my advisory committee, for many valuable comments during my Ph.D. program. Also, special thanks to Dr. A.A. Kishk, my external examiner.

I also express my deep appreciation to Dr. A. Sebak for his expert reviewing of the first manuscript of this thesis and for long hours of helpful discussions, Dr. D. Trim for many helpful discussions, and for my colleague Mr. B. Rahardjo for many academic assistances.

I would like to extend thanks to my parents, brothers and sisters for their encouragement during my education life. Special thanks to my brother, Elsayed A.M. Eid, for his constant support and encouragement to complete my graduate studies. Unfortunately, he was not able to see the completion of the thesis as he passed away five years ago. I would like to dedicate this thesis to his memory.

Special thanks are due to my wife and my dear children Ahmed, Mohammed, Alaa and Eman, for their patience and kindness during the finishing of this thesis.

The financial support by the Natural Science and Engineering Research Council of Canada in the form of Research Assistantship and also through the University of Manitoba in the form of Teaching Assistantship are appreciated. 


\section{TABLE OF CONTENTS}

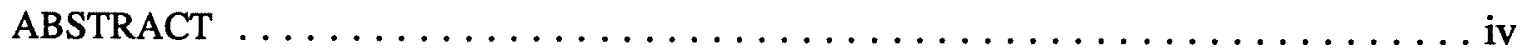

ACKNOWLEDGEMENTS $\ldots \ldots \ldots \ldots \ldots \ldots \ldots \ldots \ldots \ldots \ldots \ldots \ldots \ldots$

Table of Contents $\ldots \ldots \ldots \ldots \ldots \ldots \ldots \ldots \ldots \ldots \ldots \ldots \ldots \ldots$

List of Figures $\ldots \ldots \ldots \ldots \ldots \ldots \ldots \ldots \ldots \ldots \ldots \ldots \ldots \ldots \ldots \ldots \ldots \ldots$

List of Tables $\ldots \ldots \ldots \ldots \ldots \ldots \ldots \ldots \ldots \ldots \ldots \ldots \ldots \ldots \ldots \ldots$

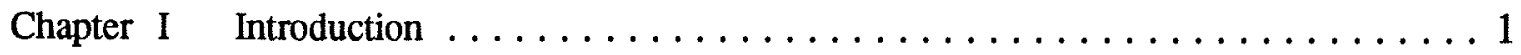

Chapter II The Field Distribution in the

Focal Plane of Conical Reflectors $\ldots \ldots \ldots \ldots \ldots \ldots \ldots$

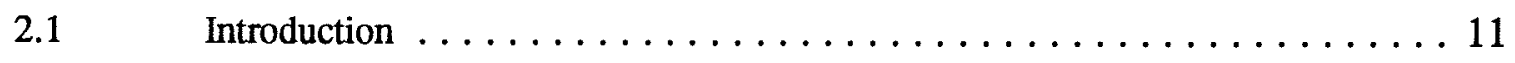

Formulation of the Focal Region Field $\ldots \ldots \ldots \ldots \ldots \ldots \ldots$

$2.3 \quad$ Numerical Results for the Focal Region Field $\ldots \ldots \ldots \ldots \ldots$ 
Chapter III Radiation from Conical Reflector

with Dipole Arrays $\ldots \ldots \ldots \ldots \ldots \ldots \ldots \ldots \ldots \ldots \ldots \ldots \ldots \ldots \ldots \ldots$

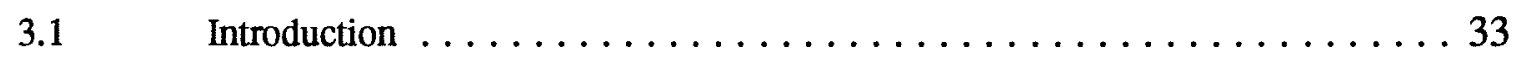

3.2 Wide Angle Cone with Linearly Polarized Feeds $\ldots \ldots \ldots \ldots \ldots \ldots$

N.3 Narrow Angle Cone with Dual Polarized Feed ............. 37

3.4 Numerical Results and Discussions for Far Field Pattern . . . . . . . 44

Chapter IV The Performance Of Conical Reflectors

with a Microstrip Comb Line Array Feed $\ldots \ldots \ldots \ldots \ldots 1$

Introduction $\ldots \ldots \ldots \ldots \ldots \ldots \ldots \ldots \ldots \ldots \ldots \ldots \ldots \ldots \ldots \ldots \ldots \ldots$

Wide Angle Cone with Linearly Polarized

with Microstrip Comb Line Array Feed $\ldots \ldots \ldots \ldots \ldots$. . . . . 63

Numerical Results and Discussions for Far Field Patterns $\ldots \ldots \ldots$. . . 68 
Chapter $\mathrm{V}$ Conclusions and Recommendations for Future Work $\ldots \ldots \ldots \ldots 3$

$5.1 \quad$ Discussion $\ldots \ldots \ldots \ldots \ldots \ldots \ldots \ldots \ldots \ldots \ldots \ldots \ldots \ldots \ldots \ldots \ldots$

$5.2 \quad$ Recommendations for Future Research Work $\ldots \ldots \ldots \ldots \ldots$

Appendix A Near Fields of a Finite Dipole oriented along the $\mathrm{x}$-axis

and fed by a Sinusoidal Current Distribution $\ldots \ldots \ldots \ldots 5$

Appendix B Near Fields of a Finite Dipole oriented along the z-axis

and fed by a Sinusoidal Current Distribution $\ldots \ldots \ldots \ldots 2$

Appendix C Design and Analysis of Microstrip Comb Line Linear

Array Antenna with known Excitation Distribution . . . . . . . . . . 94

Appendix D Radiation Pattem of Open-Circuit Rectangular

Microstrip Patch Resonator . . . . . . . . . . . . . . . 103

References . . . . . . . . . . . . . . . . . . . . . . 109 


\section{List of Figures}

Figure page

$\begin{array}{lll}1.1 & \text { Conical Gregorian geometry } & 7\end{array}$

$\begin{array}{lll}1.2 & \text { Conical Quadreflex geometry } & 8\end{array}$

1.3 Conical Line Feed geometry 9

$2.1 \quad$ The geometry of conical reflector 12

2.2 The co-polar field component $E_{x}$ on and near the focal line, $\phi=0^{0}$

2.3 The co-polar field component $E_{x}$ on and near the focal line, $\phi=0^{0}$

2.4 The co-polar field component $E_{x}$ on and near the focal line, $\phi=0^{0}$

2.5 Field distributions on a cylindrical surface of $\rho=0.25 \lambda \quad 20$

2.6 Field distributions on a cylindrical surface of $\rho=0.25 \lambda \quad 21$

2.7 Field distributions on a cylindrical surface of $\rho=0.25 \lambda \quad 22$

2.8 Phase distributions for the field components of Fig. 2.5

2.9 Phase distributions for the field components of Fig. $2.6 \quad 24$

2.10 Phase distributions for the field components of Fig. $2.7 \quad 25$

2.11 Variation of the focal region field distributions as a function of radial distance

2.12 Variation of the focal region field distributions as a function of radial distance

2.13 Variation of the focal region field distributions as a function of radial distance

2.14 Hypothetical focal region field distributions on a cylindrical surface of $\rho=0.25 \lambda$, generated by the curve fittings for data of Fig. 2.5

2.15 Hypothetical focal region field distributions on a cylindrical 
surface of $\rho=0.25 \lambda$, generated by the curve fittings for data of Fig. 2.7

2.16 Calculated co-polar field component $E_{x}$ on the focal line, $\phi=0^{0}$, by the present work (PO) and the method of moments (MOM)

2.17 Phase distributions for the calculated co-polar field component of Fig. 2.16

3.1 Conical reflector having a line source feed in the form of an array of linear dipoles, oriented parallel to the $\mathrm{x}$-axis

3.2 Conical reflector having a line source feed in the form of two orthogonal arrays of linear dipoles, polarized along $\mathrm{x}$ and $\mathrm{z}$ directions

3.3 The radiation patterns of a wide angle conical reflector having a line source feed in the form of linear dipoles oriented parallel to the $\mathrm{x}$-axis, excitation according to focal field distribution of Fig. 2.5

3.4 The radiation patterns of a wide angle conical reflector having a line source feed in the form of linear dipoles oriented parallel to the $\mathrm{x}$-axis, excitation according to hypothetical focal field distribution of Fig. 2.14

3.5 The radiation patterns of a $90^{\circ}$ conical reflector with a dual polarized line source feed of Fig. 3.2, excitation according to focal field distributions of Fig. 2.7

3.6 The radiation patterns of a $90^{0}$ conical reflector with a dual polarized line source feed of Fig. 3.2, excitation according to hypothetical focal field distributions of Fig. 2.15

3.7 The radiation patterns of a wide angle conical reflector having a line source feed in the form of linear dipoles oriented parallel to the $\mathrm{x}$-axis, excitation according to focal field 
distribution of Fig. 2.16

3.8 Variation of antenna gain with the number of array $\left(N_{x c}\right)$, and the optimal location from the apex $\left(s_{o x}\right)$

3.9a The field component $E_{x}$ over the aperture plane of wide angle conical reflector $\left(v=75.0^{\circ}\right)$, at a distance $50.0 \lambda$ from the apex

$3.9 \mathrm{~b}$ The field component $E_{y}$ over the aperture plane of wide angle conical reflector $\left(v=75.0^{\circ}\right)$, at a distance $50.0 \lambda$ from the apex

3.10a The field component $E_{x}$ over the aperture plane of wide angle conical reflector $\left(v=75.0^{\circ}\right)$, at a distance $100.0 \lambda$ from the apex

3.10b The field component $E_{y}$ over the aperture plane of wide angle conical reflector $\left(v=75.0^{\circ}\right)$, at a distance $100.0 \lambda$ from the apex

3.11a The field component $E_{x}$ over the aperture plane of wide angle conical reflector $\left(v=75.0^{\circ}\right)$, at a distance $150.0 \lambda$ from the apex

$3.11 \mathrm{~b}$ The field component $E_{y}$ over the aperture plane of wide angle conical reflector $\left(v=75.0^{\circ}\right)$, at a distance $150.0 \lambda$ from the apex

4.1a Geometry of comb line array

4.1b Geometry of a dual polarized array using a comb line and proximity coupled dipole array

4.2 Conical reflector having a line source feed in the form of two microstrip comb line arrays, oriented parallel to the $\mathrm{x}$-axis

4.3 The field distribution along the aperture of the microstrip comb line array elements, with max stub width $>15.0 \mathrm{~mm}$, 
$P_{l}$ is the percentage load power

4.4 The field distribution along the aperture of the microstrip comb line array elements, with $\max$ stub width $=15.0 \mathrm{~mm}$, $P_{l}$ is the percentage load power

4.5 The field distribution along the aperture of the microstrip comb line array elements, with $\max$ stub width $=10.0 \mathrm{~mm}$, $P_{l}$ is the percentage load power

4.6 Stub width and conductance for the field distributions of Fig. $4 ., P_{l}$ is the percentage load power

4.7 Stub width and conductance for the field distributions of Fig. $4.4, P_{l}$ is the percentage load power

4.8 Stub width and conductance for the field distributions of Fig. $4.5, P_{l}$ is the percentage load power

4.9 The radiation patterns of a wide angle conical reflector having a line source feed in the form of two microstrip comb line arrays oriented parallel to the $\mathrm{x}$-axis, excitation according to actual focal field distribution of Fig. $4.3, P_{l}$ is the percentage load power

4.10 The radiation patterns of a wide angle conical reflector having a line source feed in the form of two microstrip comb line arrays oriented parallel to the $\mathrm{x}$-axis, excitation according to hypothetical focal field distribution of Fig. $4.3, P_{l}$ is the percentage load power

4.11 The radiation patterns of a wide angle conical reflector having a line source feed in the form of two microstrip comb line arrays oriented parallel to the $\mathrm{x}$-axis, excitation according to actual focal field distribution of Fig. $4.4, P_{l}$ is the percentage load power 
4.12 The radiation patterns of a wide angle conical reflector having a line source feed in the form of two microstrip comb line arrays oriented parallel to the $\mathrm{x}$-axis, excitation according to hypothetical focal field distribution of Fig. 4.4, $P_{l}$ is the percentage load power

4.13 The radiation patterns of a wide angle conical reflector having a line source feed in the form of two microstrip comb line arrays oriented parallel to the $\mathrm{x}$-axis, excitation according to actual focal field distribution of Fig. $4.5, P_{l}$ is the percentage load power

4.14 The radiation patterns of a wide angle conical reflector having a line source feed in the form of two microstrip comb line arrays oriented parallel to the $\mathrm{x}$-axis, excitation according to hypothetical focal field distribution of Fig. 4.5, $P_{l}$ is the percentage load power

A.1 Dipole geometry for near field analysis 86

B.1 Dipole geometry for near field analysis 93

C.1 Microstrip comb line array (a) Array element geometry showing the ith stub (b\&c) T-junction and its equivalent circuit

C.2 (a) The equivalent circuit for a typical stub and its associate T-junction (b) The traveling wave array with a resistive load $G_{l}$

D.1 Sketch of microstrip line showing coordinate system and radiating apertures at $x= \pm l, 0 \ll y \ll 2 h$, $0 \ll z<<w$ 


\section{List of Tables}

Table

3.1 Variation of the calculated gains, first side lobe levels with the aperture distribution

3.2 Variation of the calculated gains, first side lobe levels with the aperture distribution 


\section{CHAPTER I}

\section{Introduction}

The widespread use of large reflector antennas, capable of high power transmission and low noise reception in satellite communication and radio astronomy, has greatly stimulated research in this particular field. The main reason for this is the requirement of a system with high gain, low side lobe level and low cross polarization for point to point communication.

At UHF frequencies and above, where pencil beams become practical, reflector antennas may be compared with other systems. The end-fire or Yagi, antennas produce relatively high gain but suffer from a narrow frequency band. Phased array antennas can be used to produce gains equal to those of reflector antennas, however, they are not universally useful, since, they have complicated structures and are costly to produce [1]. On the other hand, reflector systems have simple geometries and are usually inexpensive to fabricate. They can also be serviced mechanically with far less sophistication than phased arrays. As a single monolithic unit on a mechanically scanning assembly, a reflector is relatively inexpensive to scan. A phased array, on the other hand, requires a phase shifter for each element or at least for each subarray. Therefore, the reflecting systems are the appropriate choice for most point to point communication systems.

In reflecting systems, the reflectors are mainly used to modify the radiation from a radiating element or feed. The reflectors that are of most interest in the antenna field, can have a variety of contours like a circle, ellipse , parabola, hyperbola, etc, or can have the shaped contour designed for some specific purpose [2]. The feed for the reflecting system can either be helices, spirals, horns, etc, [3,4] or a feed consisting of one or more secondary reflectors $[5,6,7,8]$. 
The plane reflector, because of simplicity, was one of the first reflectors to be used in practice [2]. A plane reflector when placed near the radiating element reduces backward radiation and provides gain in the forward direction. To better collimate the energy in the forward direction, the geometrical shape of the plane reflector itself must be changed so as to prohibit radiation in the back and side directions. One arrangement which accomplishes this, consists of two plane reflectors joined so as to form a corner. Successful corner reflectors have produced gain as high as $14 \mathrm{~dB}$ with a single feed element [3].

The most useful of the high gain reflector antennas utilizes a paraboloidal surface. The parabolic reflector has the property of transforming the wave originating from a source at the focus into a pencil beam [9]. A parabolic reflector can take two different forms. One configuration is that of the parabolic right cylinder, whose energy is collimated at a line that is parallel to the axis of the cylinder through the focal point of the reflector [4]. The most widely used feed for this type is a linear dipole, a linear array, or a slotted waveguide. The other configuration of a parabolic reflector is formed by rotating the parabola around its axis, and is referred to as a paraboloid (parabola of revolution). With this reflector, a pencil beam is generated by using a point source feed. A corrugated conical horn has been widely utilized as a feed for this arrangement [9].

The generation of pencil-shaped antenna beam by use of a point source feed and paraboloidal reflector has reached a state of refinement such that extremely narrow beam widths, low side lobe levels and broad band operation are relatively easy to achieve. However, the beam axis coincides with the geometric axis of the paraboloidal surface so that in order to scan the beam it becomes necessary to move the entire reflector. To overcome this fundamental disadvantage, a portion of spherical reflecting surface may be used as a reflector [10]. This antenna is obtained by the rotation of a circular arc about an axis containing the center. The beam axis then coincides with the radius of the sphere upon which the feed happens to lie. Scanning is achieved by a simple rotation of the feed about the center of the sphere. However, a source located at the focus of this sphere does not provide a perfect plane wave since a paraboloidal 
surface is necessary to create a plane wave.

The departure of the wavefront from a plane wave is known as spherical aberration. Due to spherical aberration, however, a point source feed cannot be used unless the primary illumination of the reflector is confined to a relatively small zone of the spherical surface [11]. The aperture efficiency is then small and total reflector size becomes enormous relative to an equivalent paraboloid. In order to minimize the effect of spherical aberration, two general approaches have been used. The first approach involves the use of a restricted aperture and a reflector of a sufficiently large radius [10]. This approach permits a simple design and is suitable for applications requiring very wide scan angles. The second approach uses methods of compensation for spherical aberration. These methods include the use of phased line-source feeds [12], multiple-source feeds [13,14], auxiliary reflectors [15,16], or correcting lenses [17]. With these methods of compensation a larger aperture can be effectively utilized. However, this is achieved at the expense of some complexity in design and at a reduction in the useful angle of scan.

There are a great variety of modern microwave feeds. Reflector antennas, however, are used from the ultraviolet optical region of the spectrum to the high-frequency radio region. Thus, microwave feeds constitute a spectrally limited range of structures, being special cases of more general antenna feeds. In general, a feed has a specific electromagnetic purpose independent of a particular wavelength region. Implementation of the feed system may be characteristic of a particular wavelength region, but many feed techniques are not so limited, thus it is just as profitable to classify feeds by function as by type. The most common of the microwave feeds is the horn feed. The simplicity of design along with its excellent impedance, polarization, and power handling capabilities has led to wide usage of this type of feed [18]. It can be used in the pyramidal, sectoral, or conical configurations and can provide a variety of polarization by suitable design. A multiple horn feed system can be used to give beam shaping, scanning, or aberration correction [19]. 
A helix is an example of a simple feed that can be used to give circular polarization [20]. For receiving applications where power handling capability is not a factor, a helix can be used over an octave band with good impedance performance. In addition, it does not require a special feeding arrangement to give the polarization properties.

The class of frequency-independent structures such as the planar spiral, the conical spiral, and log-periodic configurations have also been used as feeds [21,22,23]. These types have extremely wide-band characteristics and as with the helix, the spiral configurations do not require special feeding arrangements to give circular polarization. However, the log-periodic and the conical spiral configurations suffer from the fact that the phase center is frequency dependent. Thus, the pattern properties of a reflecting system will suffer defocusing effects if operated over a large frequency band.

In the case of a reflector that requires a line source feed, dipole and horn arrays as well as slotted waveguides can be used $[24,25,26]$. The microstrip array, due its small size and light weight, has been used as a feed for parabolic reflector [27].

The widespread use of paraboloidal reflectors has stimulated interest in the development of feeds to improve the aperture efficiency and to provide greater discrimination against noise radiation from the ground. This can be accomplished by developing design techniques that permit the synthesis of feed patterns with any desired distribution over the bounds of the reflector, rapid cutoff at its edges and very low minor lobes in all other space. In recent years, the two main problems that concerned feed designers were aperture efficiency and low cross-polarization [28].

In the receiving mode, an ideal feed and a matched load would be one that absorbs all the energy intercepted by the aperture when uniform and linearly polarized plane waves are normally incident upon it. The feed field distribution should be made to match the focal region field structure formed by the reflecting, scattering, and diffracting characteristics of the reflector [29]. By reciprocity, an ideal feed in the transmitting mode would be one that radiates only within the solid angle of the pattern and establishes within it an identical outward traveling wave. For this ideal feed system, the transmitting and receiving mode field distributions within the focal region are 
identical with only the direction of the propagation reversed.

In the above single reflector system, the feed is usually placed in the path of the main beam, thus blocking the main aperture and making it difficult to be reached for servicing purposes. The aperture blockage by the primary feed with its supporting struts leads to scattered radiation which results in decreased antenna gain and increased side lobe and cross polarized radiation levels [30].

To improve the performance of large ground based microwave reflector antennas for satellite tracking and communication, a two-reflector system was proposed [5]. The suggested arrangement is the Cassegrain dual-reflector system, which is often utilized in the design of optical telescopes. For an accurate description of its performance, diffraction techniques should be used to take into account diffraction from the edges of the subreflector, especially when its diameter is small [31]. In general, the Cassegrain arrangement provides a variety of benefits, including: (1) the ability to place the feed in a convenient location, (2) the reduction of spillover and minor lobe radiation, (3) the ability to obtain an accurate equivalent focal length much greater than the physical length, and (4) the capability for scanning or broadening of the beam by moving one of the reflecting surfaces [2].

Suitable modifications to the geometrical shape of the reflecting surfaces have been suggested, to improve the aperture efficiency [32,33,34]. The reshaping of the reflecting surfaces is used to generate desirable amplitude and phase distributions over one or both of the reflectors. The resultant system is usually referred to as a shaped dual-reflector.

The classical Cassegrain system may be easily extended to include a variety of forms by varying the curvatures of the two reflectors. The common variety of Cassegrain system is the Gregorian system. The Gregorian system is similar to the Cassegrain system in that a parabolic reflector is used, however the subreflector is elliptic in shape rather than hyperbolic. As in the Cassegrain the performance of the system can be evaluated in terms of an equivalent parabola [9]. The Gregorian system is commonly used in radio astronomy with simultaneous multiple frequency feeds. 
The aperture blockage in the rotationally symmetric systems mentioned above can be removed by either using an offset system [30,35] or supporting the main reflector or the subreflector with a dielectric cone $[36,37]$. The offset fed reflector antenna has become increasingly attractive for satellite communications where low side lobe levels are essential to achieve good isolation between adjacent high gain beams operated over the same frequency band. To meet these requirements, the use of offset reflectors is becoming desirable since the severe limiting effects of aperture blockage on front fed paraboloidal reflectors can be entirely removed [38]. The offset system has the advantage of reducing the mutual reaction between the reflector and the primary feed. It also makes it possible to use a larger focal length to diameter ( $\mathrm{f} / \mathrm{d}$ ) ratio while maintaining acceptable structural rigidity. As a result, offset reflector primary feeds employ a larger radiating aperture, which in certain cases can improve the shaping of the primary pattern and give a better suppression of the cross polarized radiation emanating from the feed [30].

A conical reflector has a simple geometry and focuses a point at infinity, to a line along its axis [39]. For finite reflectors, the size and axial location of the line depends on the cone angle and size. It falls between the cone apex and the aperture when the contained cone angle becomes $90^{\circ}$ [40]. Because the line source is large in size, and is difficult to design, conical reflectors have not found a wide spread application in communications. However, they provide certain advantages in the simplicity of the geometry and mechanical alignment. A large, high precision, furlable surface can be obtained at relatively low cost. Their performance has not been studied systematically, but a few studies have been reported in the literature to address their performance. The most extensive work on these reflectors is reported by Ludwig $[41,42]$, who has studied their performance experimentally using a line source feed and a Gregorian subreflector. Aperture efficiencies in the order of $60 \%$ are achieved. With a Gregorian sub-reflector having a paraboloidal profile as shown in Fig. 1.1, the structure can be illuminated by a point source using a corrugated conical horn via a subreflector (generated by a parabolic arc). However, the subreflector size is normally large and causes excessive aperture blockage, resulting in loss of efficiency and high side lobes. 


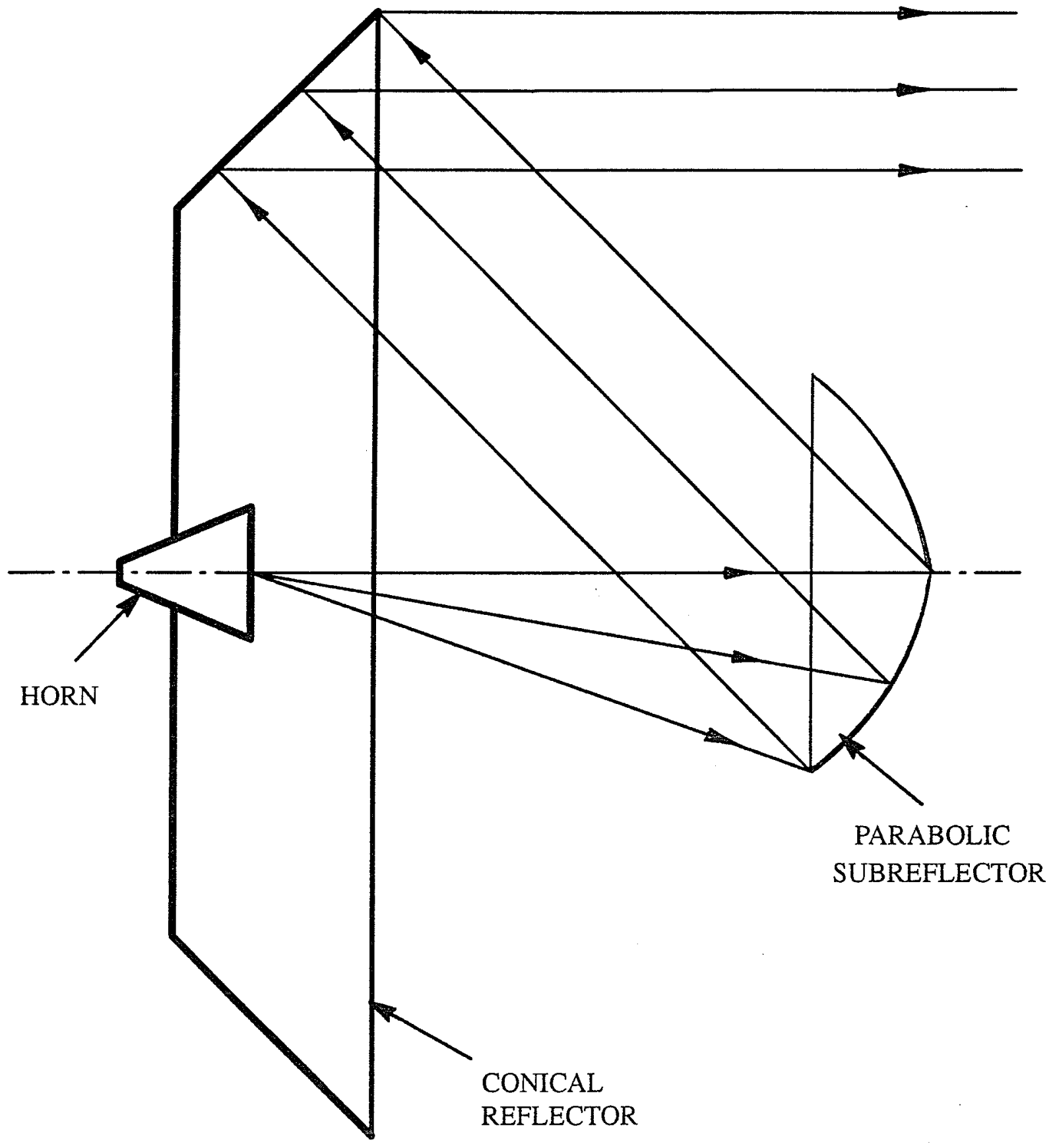

Fig. 1.1 : Conical Gregorian geometry 


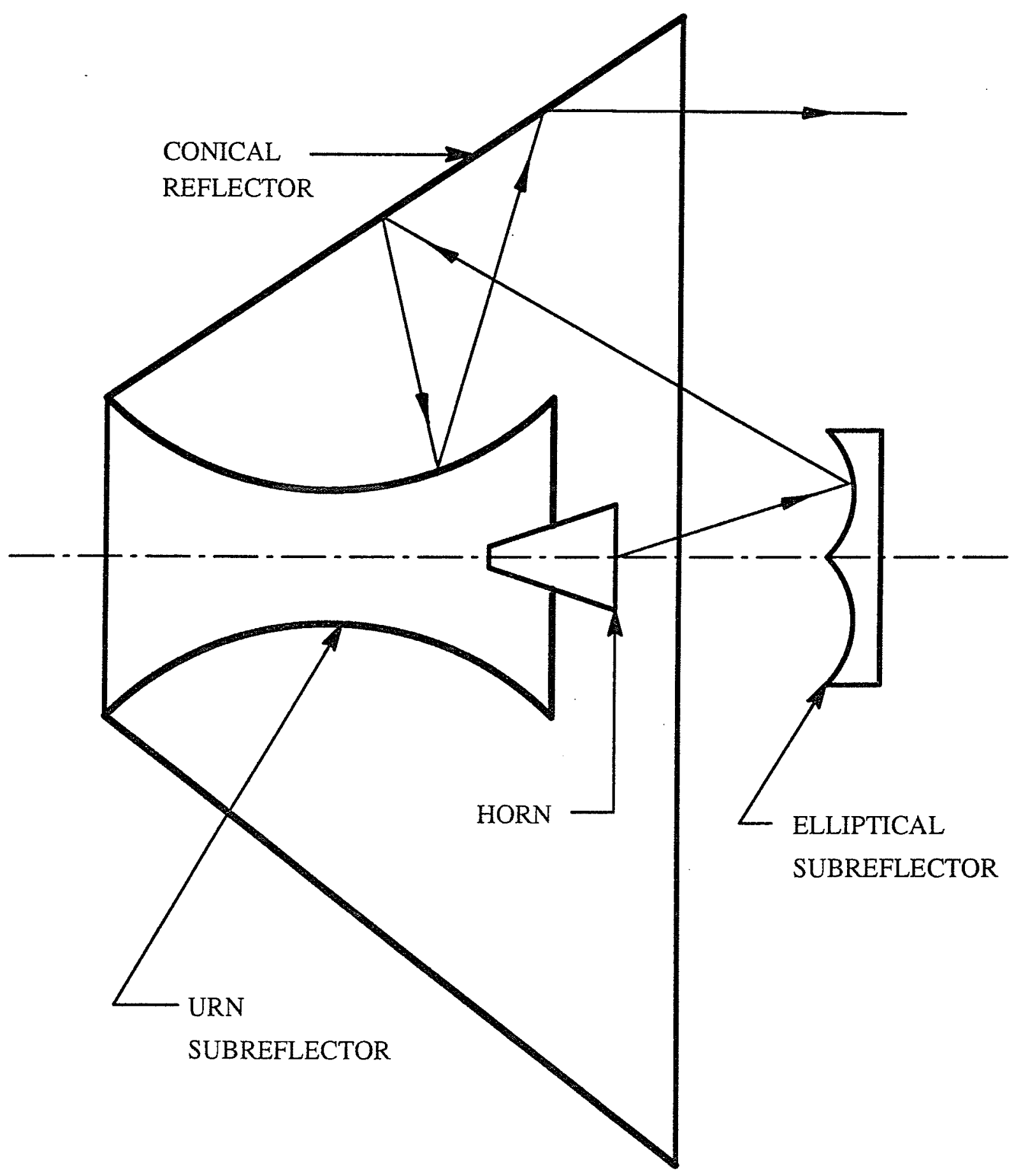

Fig. 1.2 : Conical Quadreflex geometry 


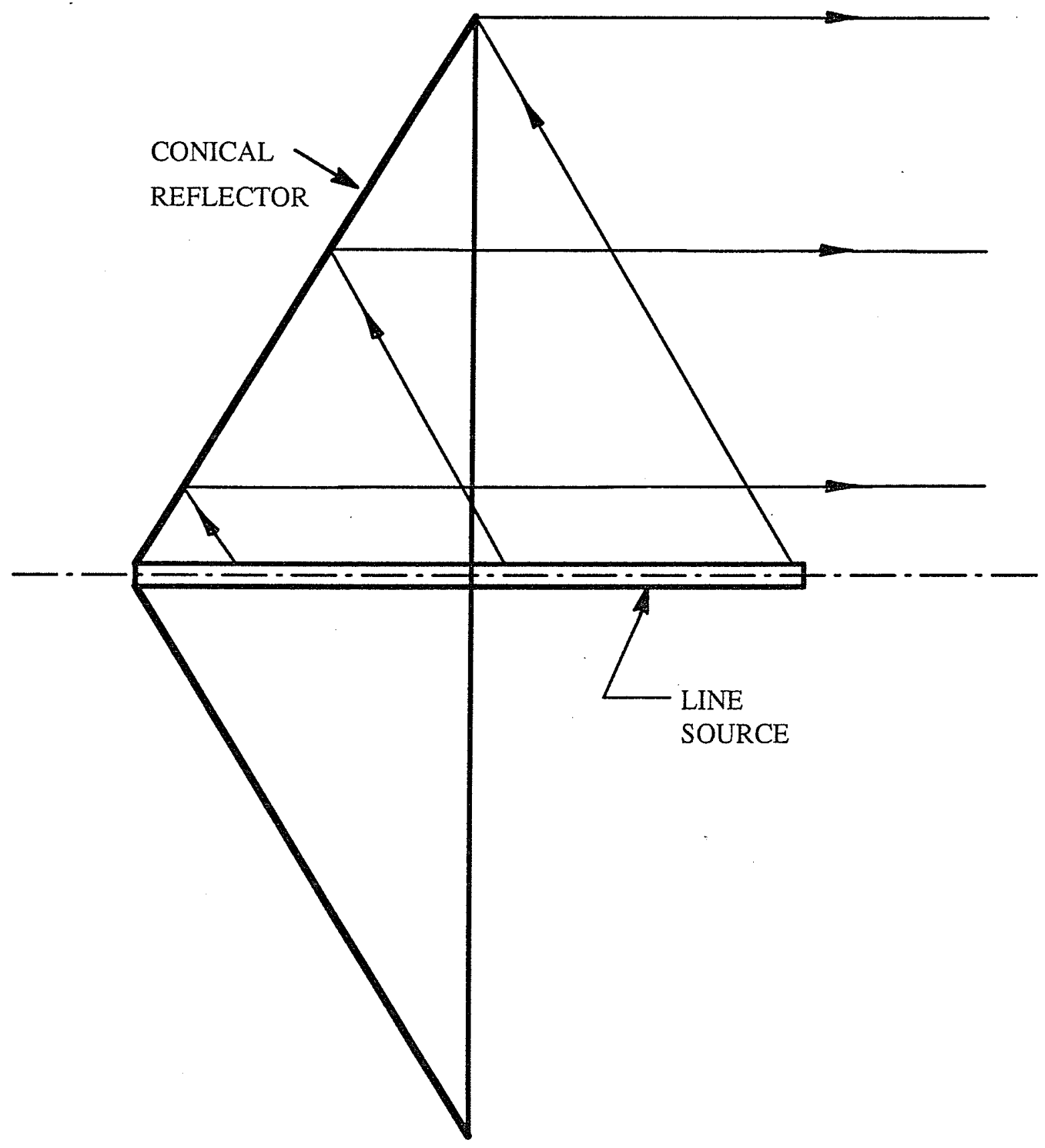

Fig. 1.3 : Conical Line Feed Geometry 
The conical-quadreflex design [43] was conceived to reduce the subreflector size. The conical reflector in this design as shown in Fig. 1.2, is illuminated by a corrugated conical horn via two subreflectors, one elliptic-shaped and the other urn-shaped. The reduced subreflector size (diameter) significantly decreases the blockage loss but also degrades the capability for multiple operations. Since the design requires multiple reflections on the conical surface, the surface losses are increased. Finally, the conical reflector fed by a line source feed is shown in Fig. 1.3. The feed consists of a uniform length of circular waveguide with radiating holes and fin phase correctors [44]. The feed utilizes the radiation principles upon which a line feed constructed for the Arecibo spherical reflector was based [26]. The conical-line-source design not only minimizes the blockage and surface losses, but also reduces the antenna weight. This approach has two short comings : narrow bandwidth, and excessive ohmic losses due to the length of the waveguide. The line source feed must provide an appropriate reflector illumination to generate the desirable far field patterns. The requirements of such a line source can be determined from the focal region field analysis, which in turn can be used in its design $[45,46]$.

The purpose of this thesis is to investigate conical reflector antennas in a systematic way. This is accomplished by using the matched feed concept to determine the electrical characteristics of a line source feed for conical reflector antennas. The performance of conical reflectors with a line source feed in the form of an array of dipoles of different configurations have been investigated. Also, The performance of conical reflectors with a microstrip comb line array has been investigated.

In chapter II, the matched feed concept is used to determine the electrical characteristics of a line source feed for conical reflector antennas. The performance of conical reflectors with a line source feed in the form of an array of dipoles of different configurations are presented in chapter III. In chapter IV, the performance of conical reflectors with a line source feed in the form of two microstrip comb line arrays are presented. Finally, conclusions of the work presented herein are drawn and recommendation for future work suggested in chapter V. 


\section{CHAPTER II}

\section{The Field Distribution in the Focal Plane of Conical Reflectors}

\subsection{Introduction}

The matched feed concept is used to determine the electrical characteristics of a line source feed for conical reflector antennas. In the matched feed approach, the reflector is illuminated by the desired far field, as a receiving antenna, and its focal region field is determined by computing the scattered field [28,29]. From reciprocity, the ideal feed aperture distribution must match this focal region field. A linearly polarized plane wave incident on the reflector is used to determine the field distribution along and in the vicinity of the focal axis of the reflector. The co-polar, cross-polar and the axial field components are determined and their dependence on the reflector size, the cone angle and the radial distance is studied.

\subsection{Formulation of the focal region field}

The focal region field distribution depends on the type of incident field and the reflector parameters. Here, we determine the focal region field, using a physical optics current distribution method. Since the reflector geometry is symmetric, a linearly polarized incident field provides adequate information on the focal region field $[28,47]$. We therefore select the reflector of Fig. 2.1, and illuminate it axially with a linearly polarized plane wave. The incident electric and magnetic fields from the plane wave have only the $\mathrm{x}$ and $\mathrm{y}$ components respectively, which can be written as :

$$
E_{x}^{i n c}=k Z_{0} e^{j k z}
$$




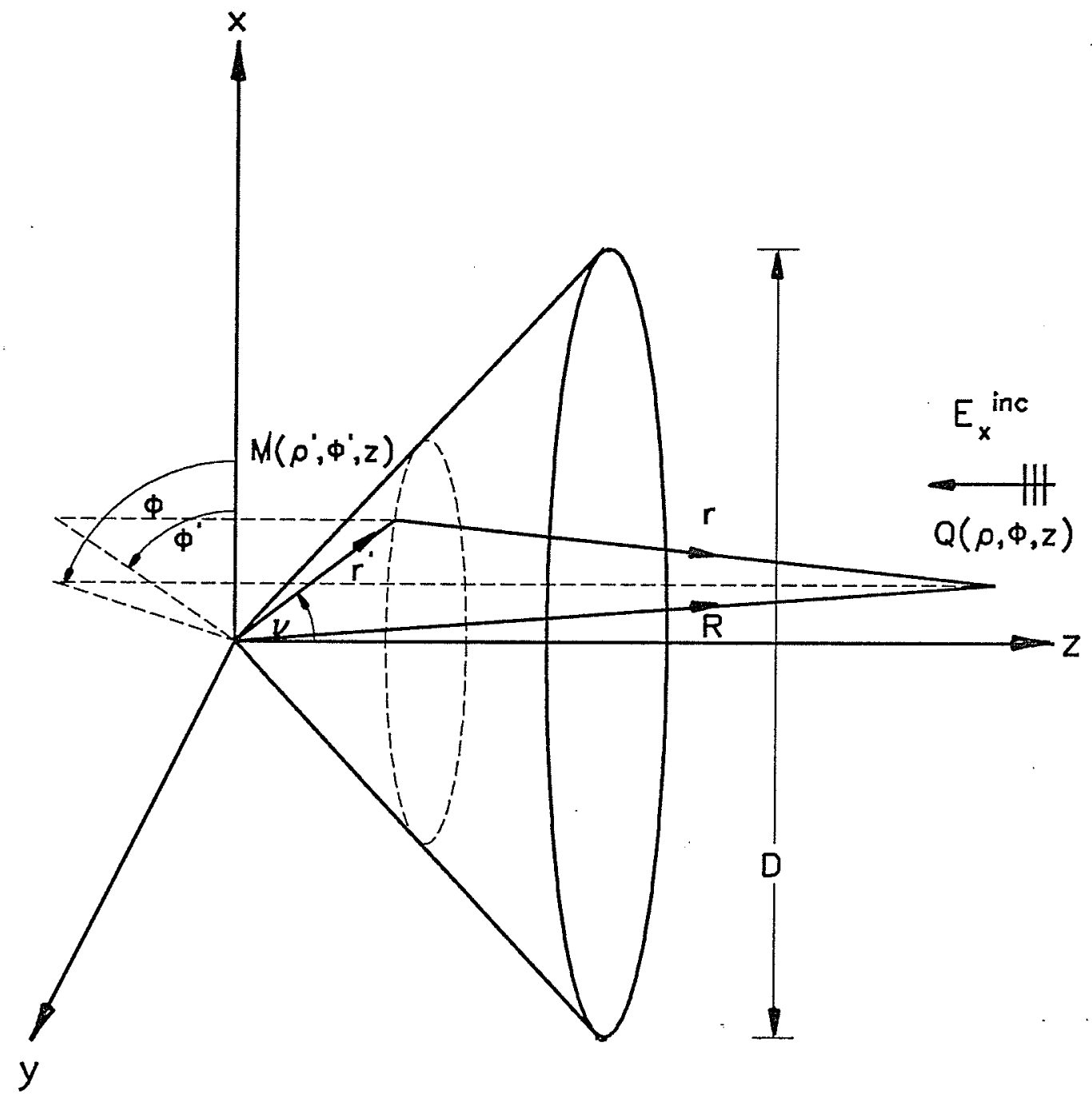

Fig. 2.1: The geometry of the conical reflector. 


$$
H_{y}^{i n c}=-k e^{j k z}
$$

where $k$ and $Z_{0}$ are the wave number and the intrinsic impedance of free space. The time dependence $e^{j \omega t}$ is suppressed throughout. The incident magnetic field $H_{y}$ induces current distributions on the conducting cone.

The field radiated by the induced current distributions, $\mathbf{J}$, can be written as [48] :

$$
\mathbf{E}^{s}=\frac{-j Z_{0}}{4 \pi k} \int_{s}\left\{Y_{1} \mathbf{J}+Y_{2}\left[\mathbf{J} \cdot \hat{a}_{r}\right] \hat{a}_{r}\right\} \Phi d s^{\prime}
$$

in which $\hat{a}_{r}$ is a unit vector from the source point $\mathrm{M}$ to the field point $\mathrm{Q}$, and

$$
\begin{aligned}
Y_{1} & =k^{2}-\frac{1}{r}\left(j k+\frac{1}{r}\right) \\
Y_{2} & =-k^{2}+\frac{3}{r}\left(j k+\frac{1}{r}\right) \\
\Phi & =\frac{e^{-j k r}}{r} \\
r & =\left[\left(\rho-\rho^{\prime}\right)^{2}+\left[z-\rho^{\prime} \cot v\right]^{2}+4 \rho \rho^{\prime} \sin ^{2}\left[\frac{\phi-\phi^{\prime}}{2}\right]\right]
\end{aligned}
$$

In these equations $(\rho, \phi, z)$ and $\left(\rho^{\prime}, \phi^{\prime}, z^{\prime}\right)$ are the the position vectors of the field and the source points, respectively.

The surface currents $\mathbf{J}$ can be determined using the physical optics approximation as :

$$
\mathbf{J}=2\left(\hat{n} \times \mathbf{H}^{i n c}\right)
$$

where $\hat{h}$ is the outward unit normal to the reflector surface, which can be written as :

$$
\hat{h}=-\cos v \cos \phi^{\prime} \hat{a}_{x}-\cos v \sin \phi^{\prime} \hat{a}_{y}+\sin v \hat{a}_{z}
$$

in which $\hat{a}_{x}, \hat{a}_{y}$ and $\hat{a}_{z}$ are unit vectors in the direction of the Cartesian coordinates $(\mathrm{x}, \mathrm{y}, \mathrm{z})$. 
Substituting (2.2) and (2.6) into (2.5), one obtains, the components of the surface current density as :

$$
\begin{aligned}
& J_{x}=2 k \sin v e^{j k \rho^{\prime} \cot v} \\
& J_{y}=0.0 \\
& J_{z}=2 k \cos v \cos \phi^{\prime} e^{j k \rho^{\prime} \cot v}
\end{aligned}
$$

Substituting (2.7) into (2.3), we obtain the components of the scattered field as :

$$
\begin{gathered}
E_{x}=\frac{-j Z_{0}}{2 \pi} \int_{0}^{D / 2} \int_{0}^{2 \pi}\left\{Y_{1}+Y_{2}\left(\frac{1}{r^{2}}\right)\left[\rho \cos \phi+\left[z \cot v-\rho^{\prime}\left(1+\cot ^{2} v\right)\right] \cos \phi^{\prime}\right]\right. \\
\left.\cdot\left(\rho \cos \phi-\rho^{\prime} \cos \phi^{\prime}\right]\right\} \Phi e^{j k \rho^{\prime} \cot v} \rho^{\prime} d \rho^{\prime} d \phi^{\prime} \\
E_{y}=\frac{-j Z_{0}}{2 \pi} \int_{0}^{D / 2} \int_{0}^{2 \pi}\left\{Y_{2}\left(\frac{1}{r^{2}}\right)\left[\rho \cos \phi+\left[\cot v-\rho^{\prime}\left(1+\cot ^{2} v\right)\right] \cos \phi^{\prime}\right]\right. \\
\left.\cdot\left(\rho \sin \phi-\rho^{\prime} \sin \phi^{\prime}\right]\right\} \Phi e^{j k \rho^{\prime} \cot v} \rho^{\prime} d \rho^{\prime} d \phi^{\prime} \\
E_{z}=\frac{-j Z_{0}}{2 \pi} \int_{0}^{D / 2} \int_{0}^{2 \pi}\left\{Y_{1} \cot v \cos \phi^{\prime}+Y_{2}\left(\frac{1}{r^{2}}\right)\left[\rho \cos \phi+\left[z \cot v-\rho^{\prime}\left(1+\cot ^{2} v\right]\right] \cos \phi^{\prime}\right]\right. \\
.(z-\rho \cot v)\} \Phi e^{j k \rho^{\prime} \cot v} \rho^{\prime} d \rho^{\prime} d \phi^{\prime}
\end{gathered}
$$

which may be used to compute the focal region field. Idealy, one should compute these field components on the cone axis, which is also the focal line. However, a practical feed will have a finite radius and the field distribution must be computed on its surface, which in most cases is a cylinder. In the following section, we provide the 
computed field components on the cone axis and its vicinity to investigate their dependence on the cone parameters and the feed radius.

\subsection{Numerical results for the focal region fields}

The components of the scattered field, from equations (2.8) to (2.10), are computed to determine the nature of the focal field distribution on and near the cone axis. Figs. 2.2, 2.3, and 2.4 show the behavior of the normalized co-polar field component, i.e. $E_{x}$, for a reflector with an aperture diameter of $30.0 \lambda$, along the cone axis ( $\rho=0$ ) and on cylindrical surfaces, representing a line source of finite radius, for various cone angles. The field distributions are similar, but their intensity decreases with increasing $\rho$. On the cone axis, i.e. on the focal line, the cross-polar component $E_{y}$, and the axial field component $E_{z}$ are both zero. Thus only the co-polar component $E_{x}$ has a non zero value. Similarly in the $H$-plane, $\left(\phi=90^{\circ}, \rho=0.0\right)$, both $E_{y}$ and $E_{z}$ components are zero, but $E_{x}$ has a non zero value. The field distributions on a cylinder of radius $0.25 \lambda$, about the cone axis, are shown in Figs. 2.5, 2.6, and 2.7 which are normalized with respect to the co-polar peak intensity on the cone axis shown in Figs. $2.2,2.3$, and 2.4, respectively. For the wide angle cone, in Fig. 2.5, both the crosspolar $E_{y}$ and axial component $E_{z}$ are small. However, when the cone angle reduces to $125^{\circ}$ in Fig. 2.6, the cross-polar field component remains small, but the axial field component $E_{z}$ becomes significant. Decreasing the cone angle further to $90^{\circ}$ in Fig. 2.7 , both cross- polar and axial components increase rapidly and the latter one exceeds the co-polar component $E_{x}$. Figs. 2.8, 2.9, and 2.10 show the phase distributions of the field components shown in Figs. 2.5, 2.6, and 2.7, respectively. For large cone angles, the phase varies rapidly, but as the cone angle decreases to $90^{\circ}$, its rate of change reduces to zero. In the latter case, the co-polar and cross-polar components have similar phase distributions, but the axial component is in phase quadrature.

The variation of the focal region field distributions as a function of the radial distance for various cone angles of $150^{\circ}, 125^{\circ}$, and $90^{\circ}$ and for different locations along the focal line are shown in Figs. 2.11, 2.12, and 2.13, respectively. Both $E_{x}$ and $E_{z}$ 
components are computed in the $E$-plane, $\left(\phi=0^{\circ}\right)$, while the $E_{y}$ component is calculated in the $\phi=45^{\circ}$ plane, where they have maximum distributions. Their relative magnitude and variation with the cone angle are similar to the previous cases discussed above. These figures also show that near the axis the co-polar field component $E_{x}$ decreases with $\rho$, but other field components increase. This indicates that in design of practical line sources that have a finite radius, the cross-polar and axial field components must be finite to cancel the reflector generated cross-polar fields.

Since the focal region fields have relatively complex distributions, with excessive amount of oscillations, design of line sources with corresponding excitations would be impractical. The line source designs would become considerably simpler by selecting smoother distributions. Consequently, the original focal field distributions are also used to generate, hypothetical, but smooth distributions, using a curve fitting approach [49]. Two such examples are shown in Figs. 2.14 and 2.15 that correspond to the focal field distributions of Figs. 2.5 and 2.7. They are also used later in this study to investigate the conical reflector performance.

In Fig. 2.16, the calculated co-polar field component, i.e. $E_{x}$, for a reflector with an aperture diameter of $30.0 \lambda$, along the cone axis $(\rho=0)$ is plotted. The results obtained using the present work (PO) is compared with the numerical solution based on the method of moments (MOM) [50].The agreement demonstrated in Fig. 2.16, is found to be reasonable. For the phase distributions shown in Fig. 2.17, the (PO) and (MOM) methods are in good agreement. 


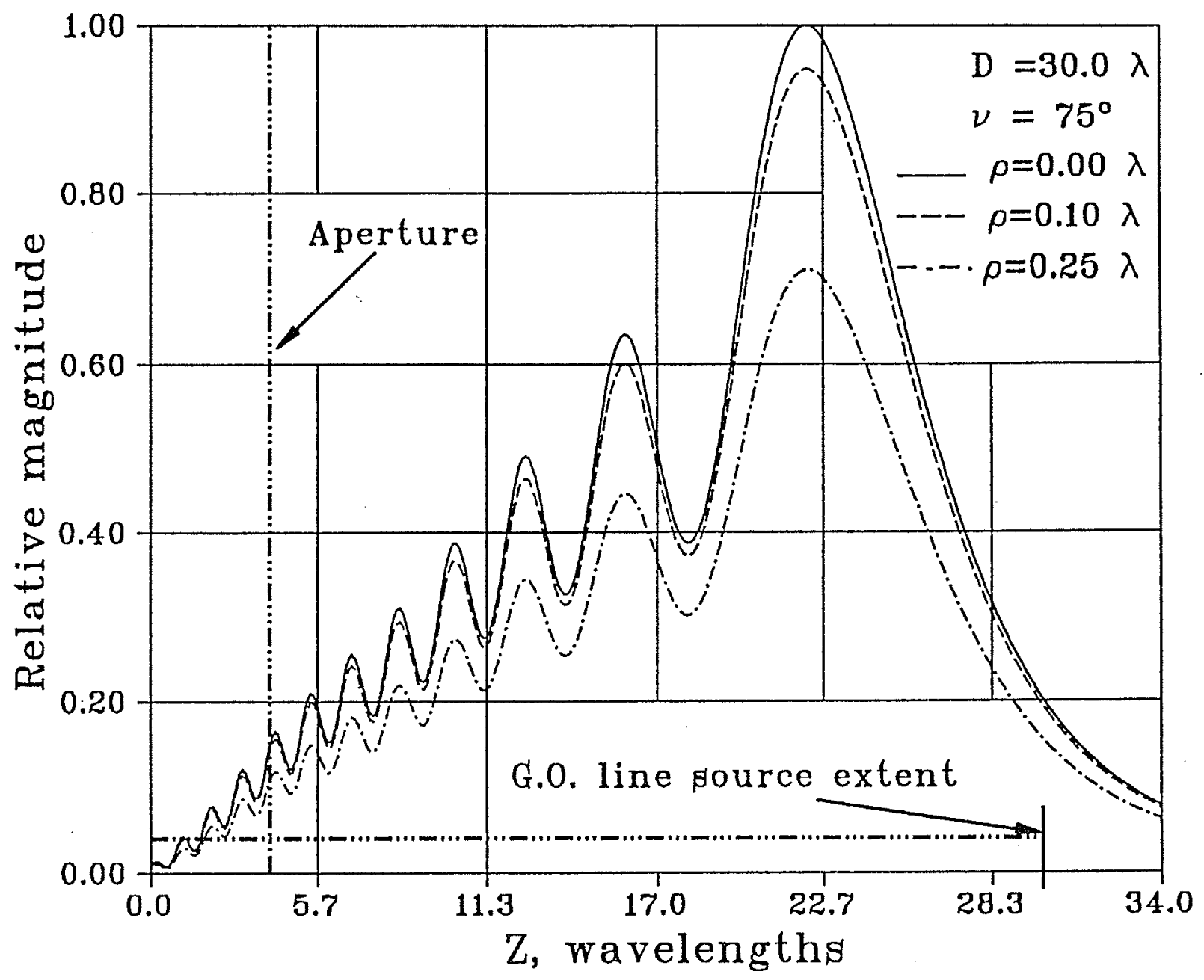

Fig. 2.2 : The co-polar field component $E_{x}$ on and near the focal line, $\varphi=0^{\circ}$. 


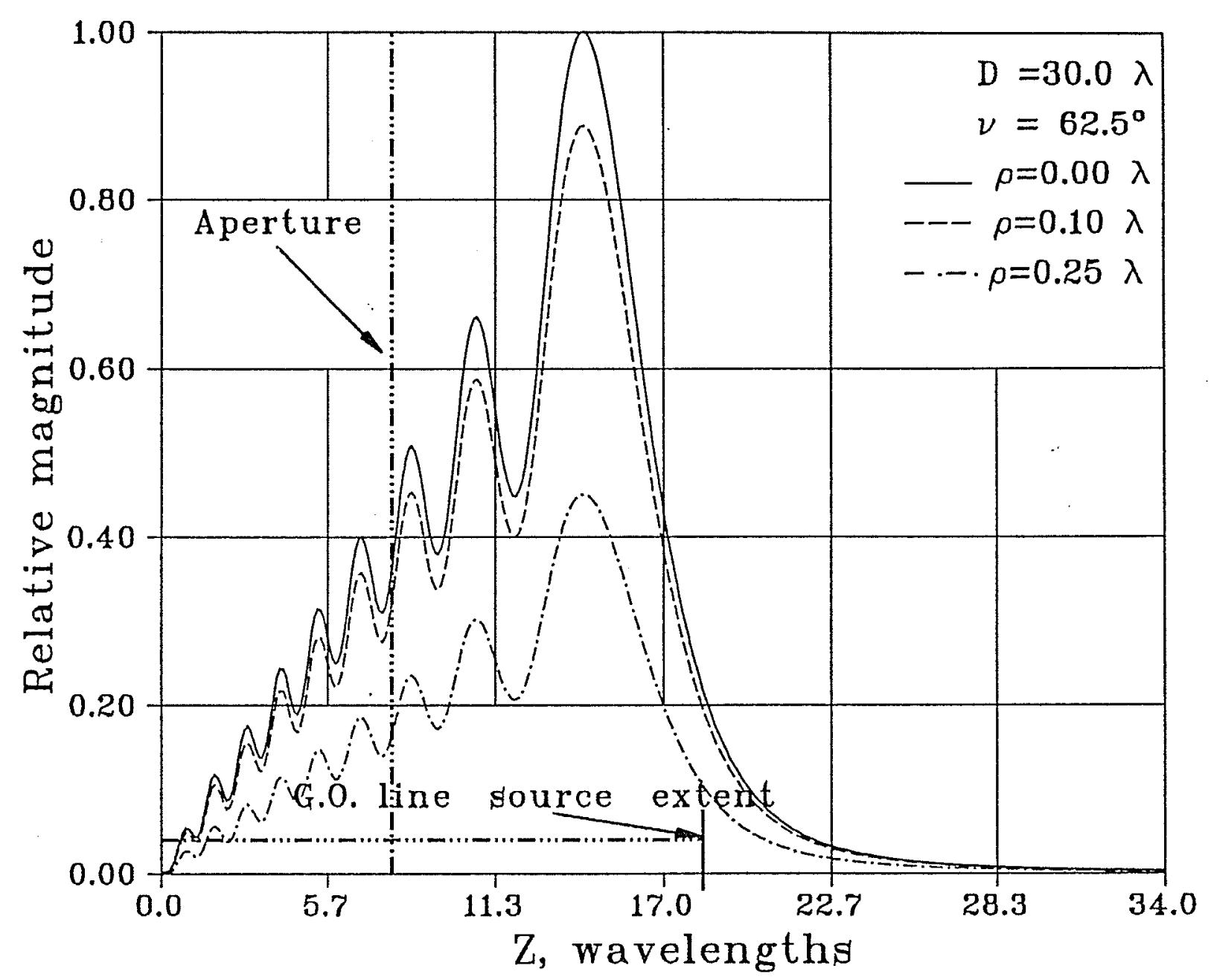

Fig. 2.3 : The co-polar field component $E_{x}$ on and near the focal line, $\varphi=0^{\circ}$. 


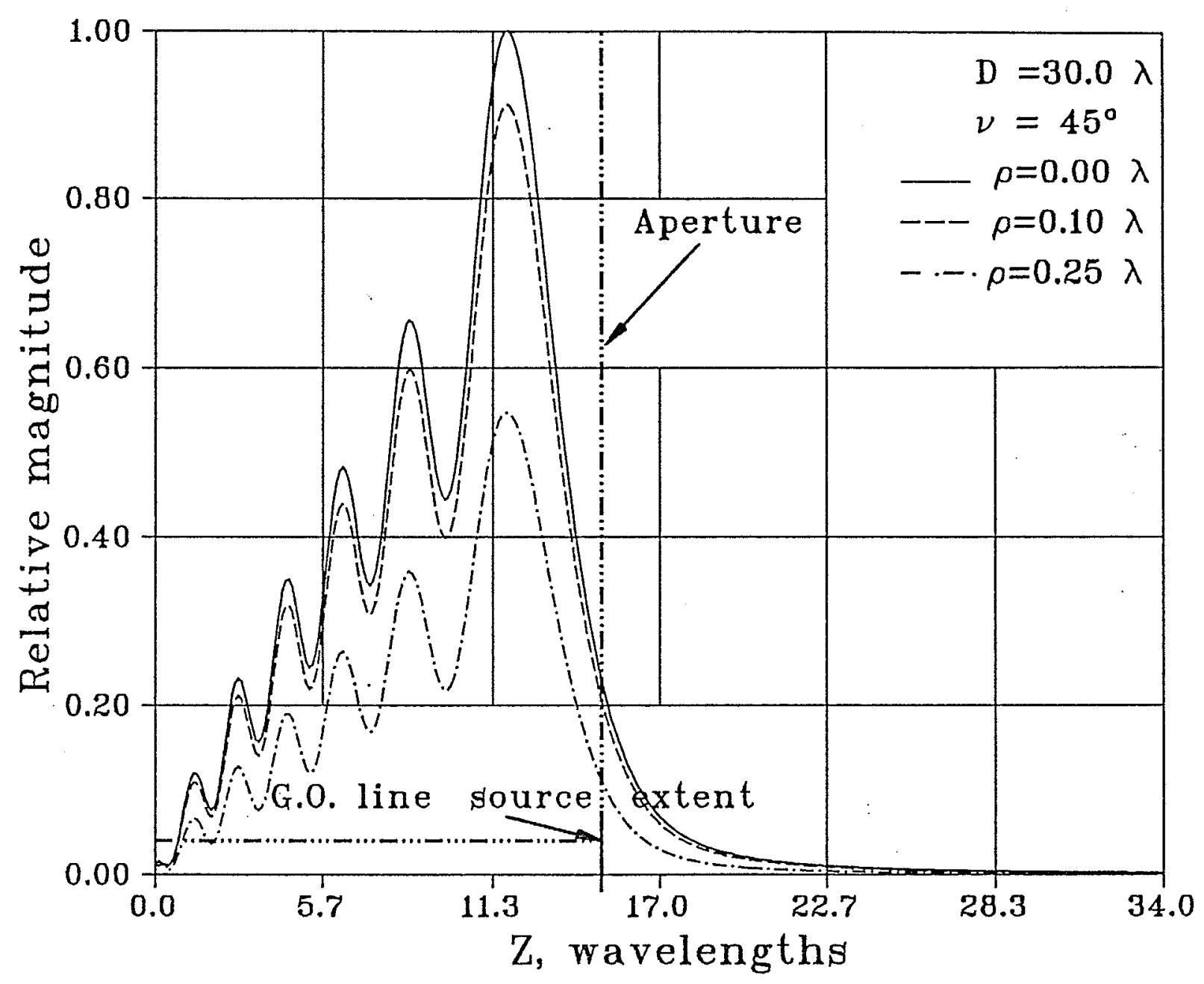

Fig. 2.4 : The co-polar field component $\mathrm{E}_{\mathrm{x}}$ on and near the focal line, $\varphi=0^{\circ}$. 


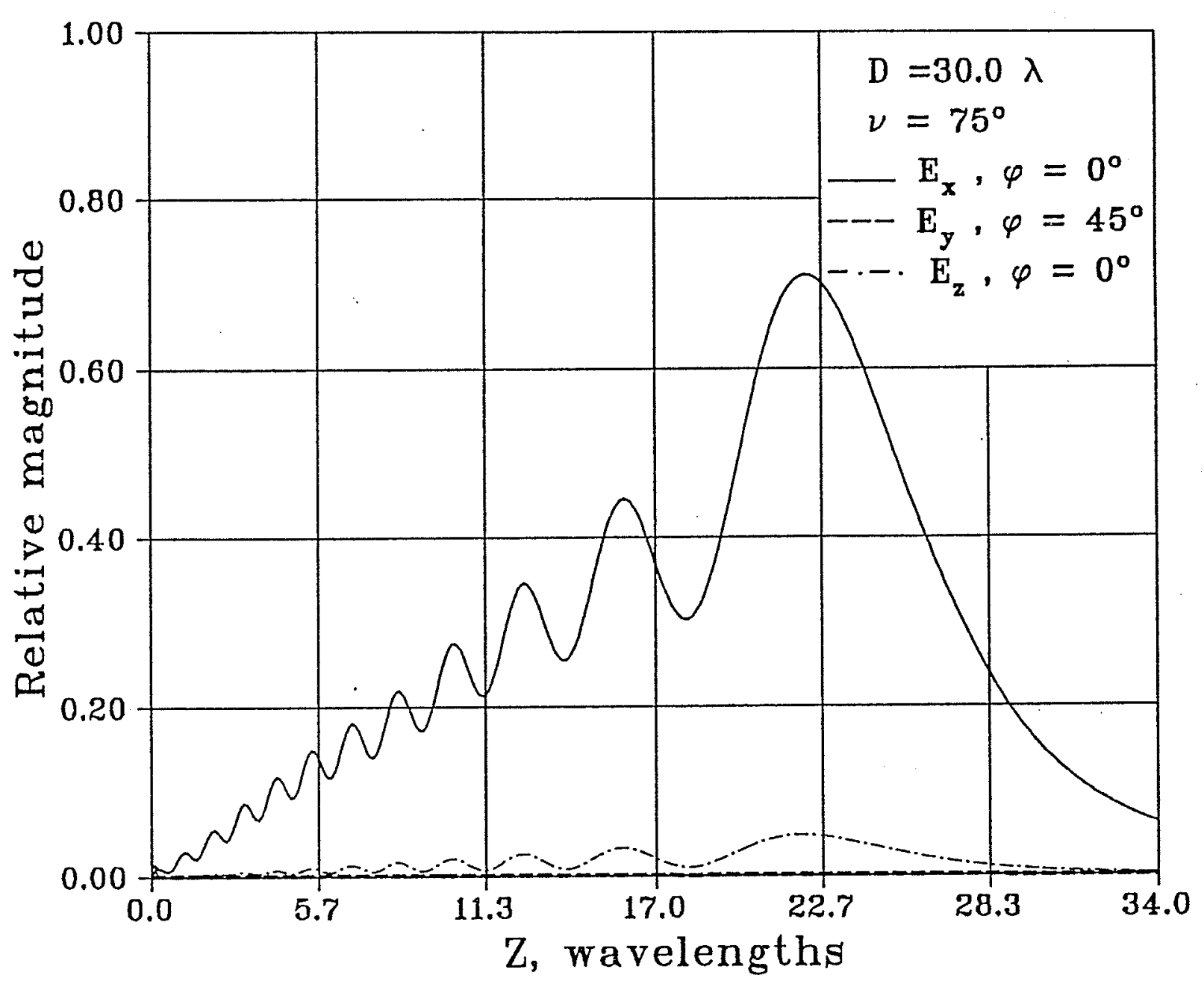

Fig. 2.5 : Field distributions on a cylindrical surface of $\rho=0.25 \lambda$. 


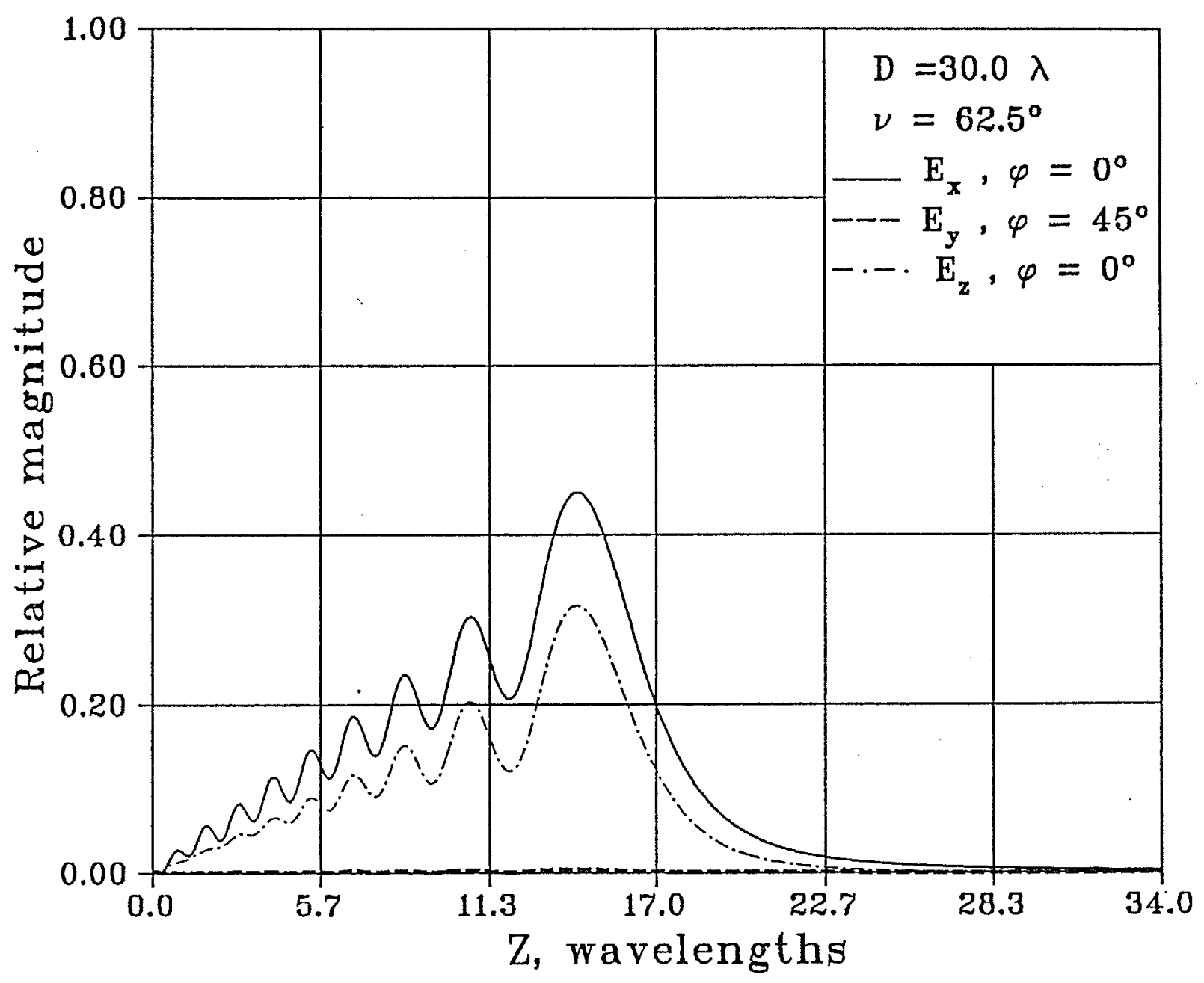

Fig. 2.6 : Field distributions on a cylindrical surface of $\rho=0.25 \lambda$. 


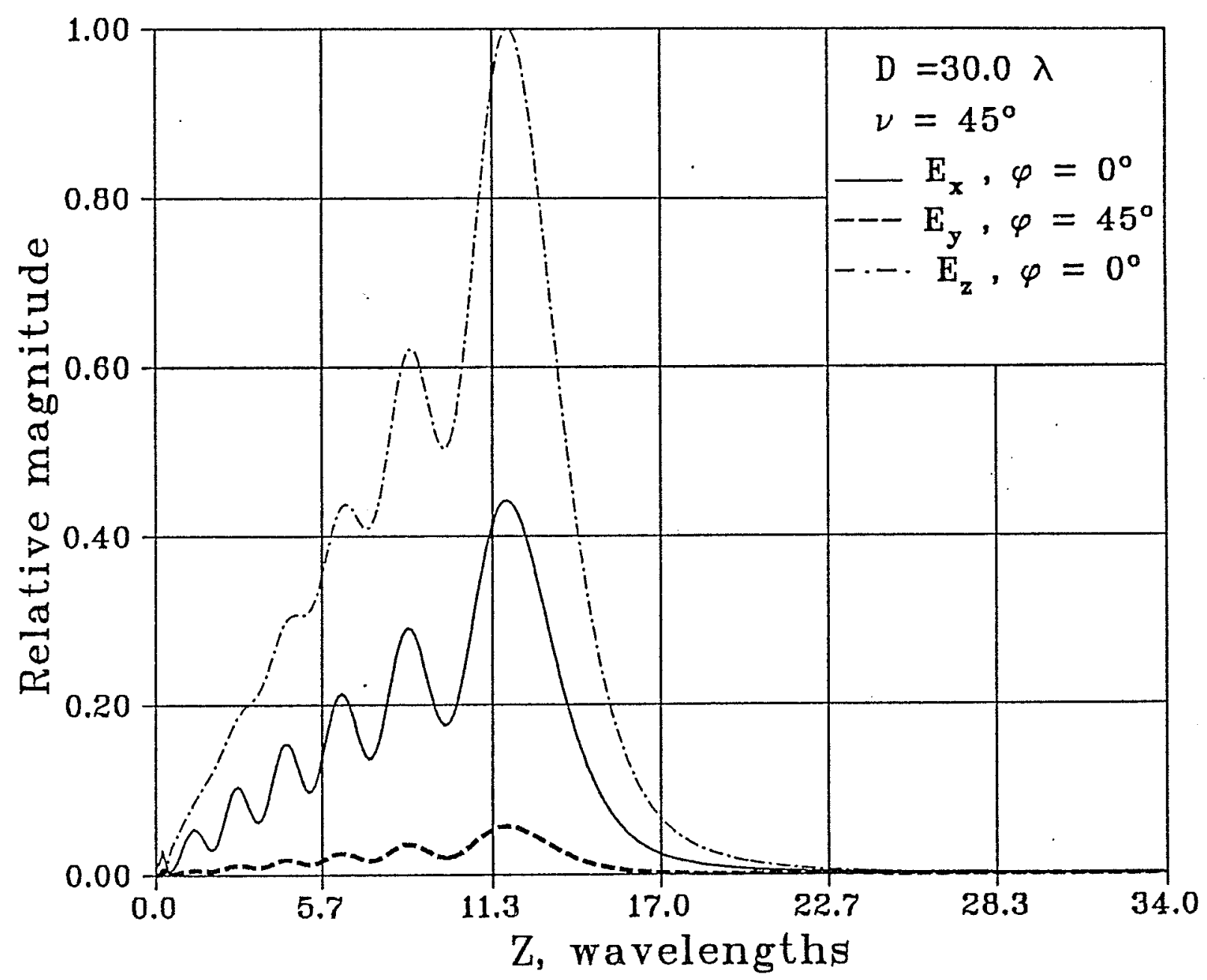

Fig. 2.7 : Field distributions on a cylindrical surface of $\rho=0.25 \lambda$. 


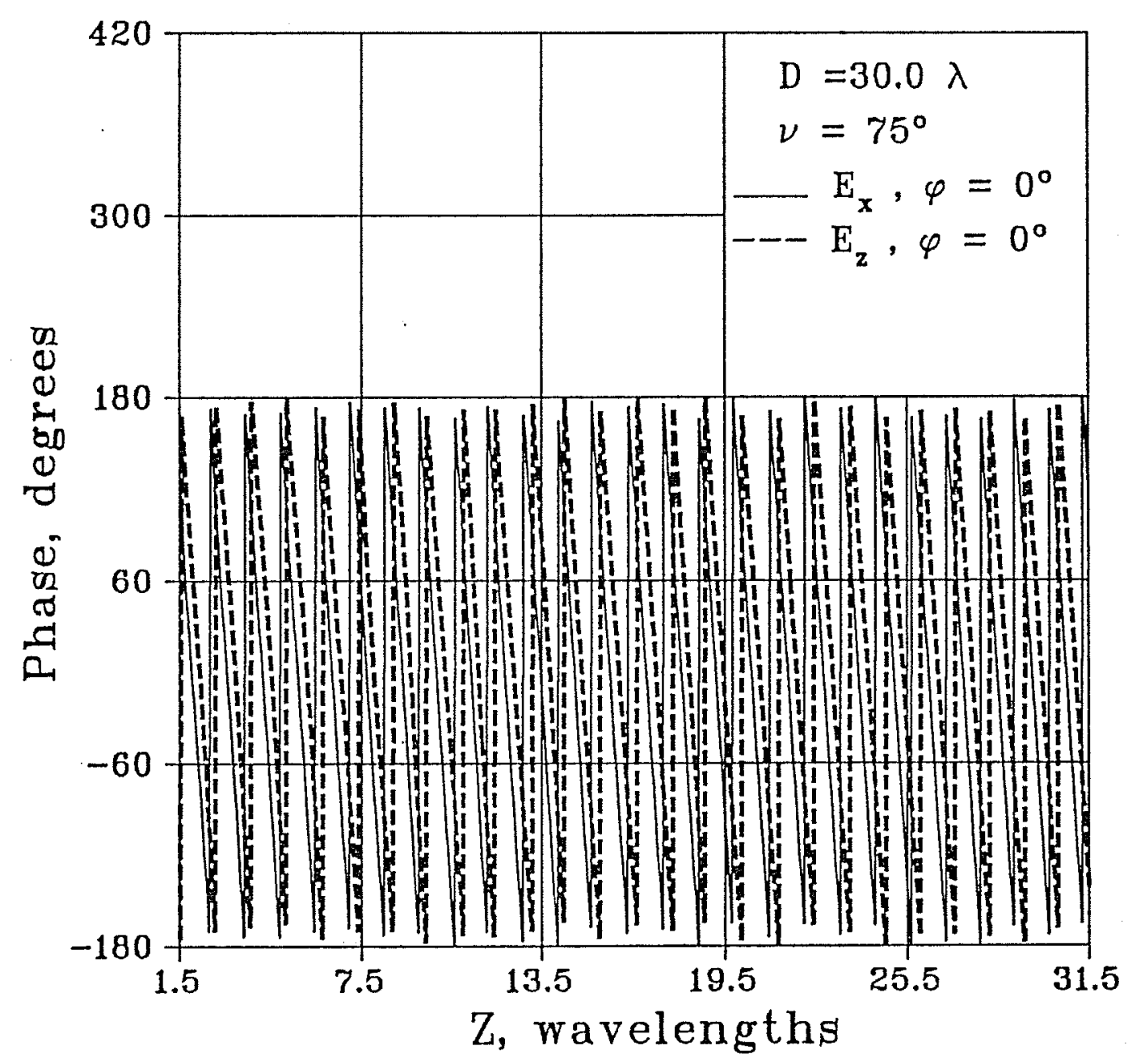

Fig. 2.8 : Phase distributions for the

field components of Fig. 2.5. 


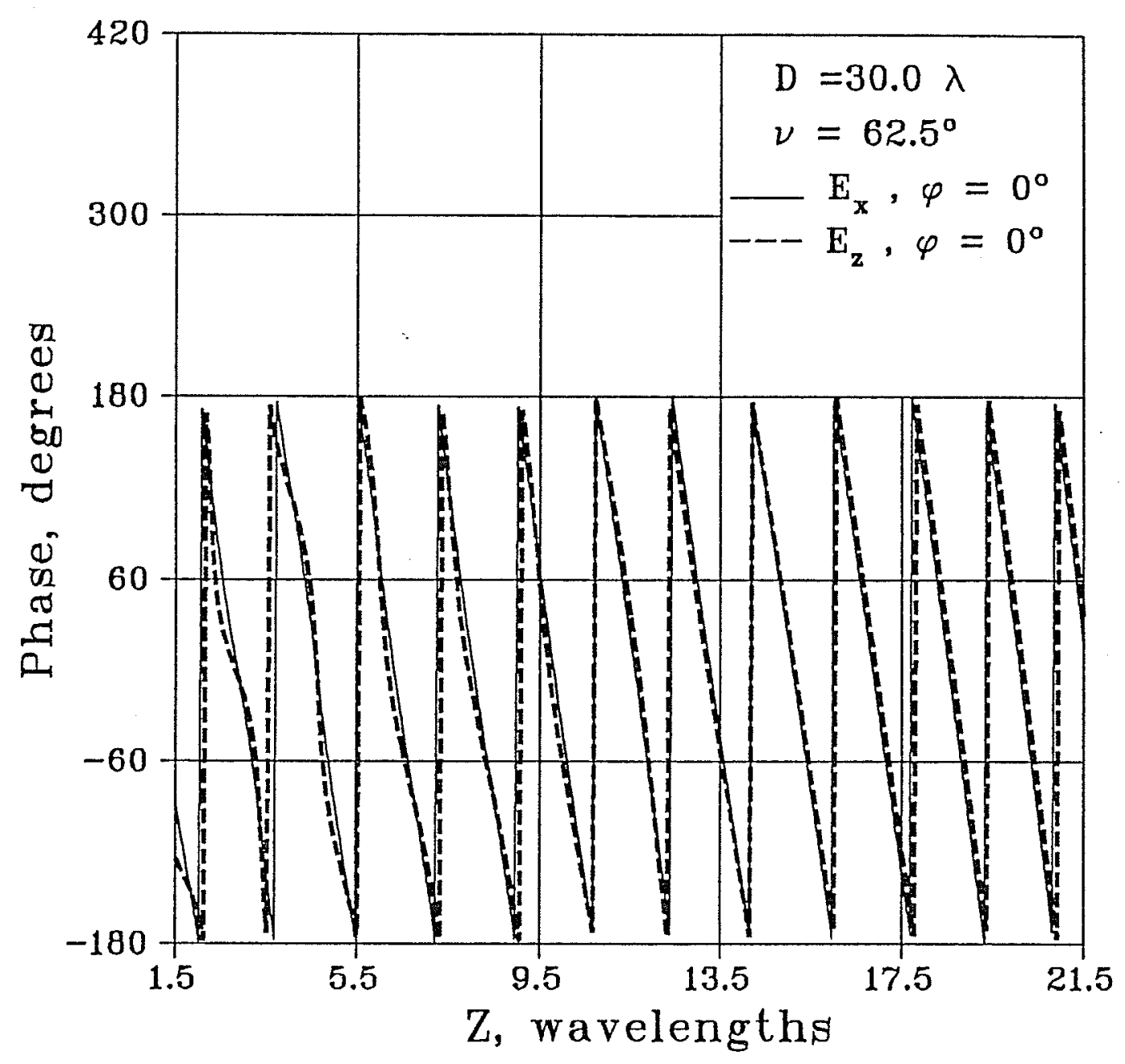

Fig. 2.9 : Phase distributions for the field components of Fig. 2.6. 


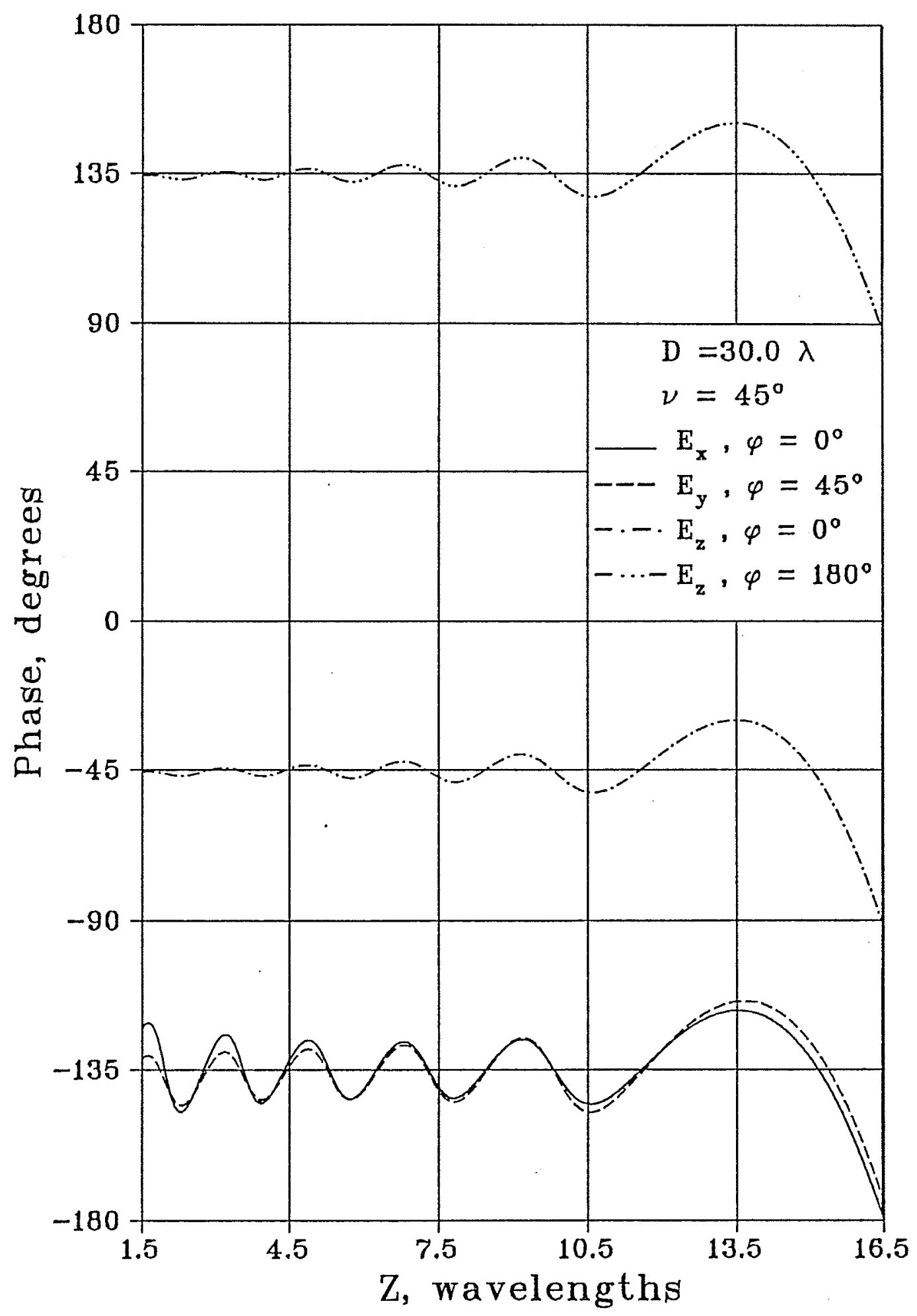

Fig. 2.10 : Phase distributions for the field components of Fig. 2.7. 


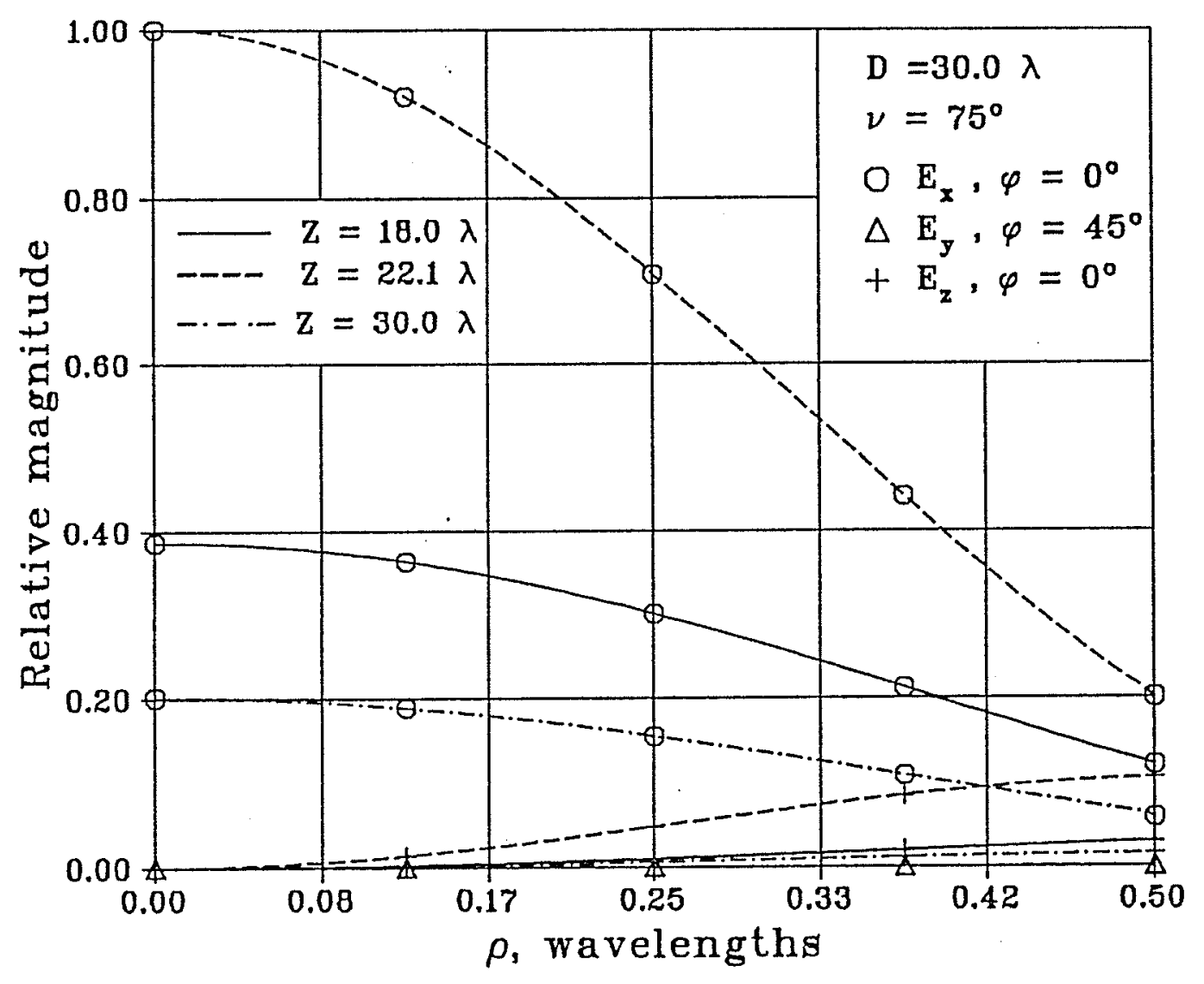

Fig. 2.11 : Variation of focal region field distributionf as a function of radial distance. 


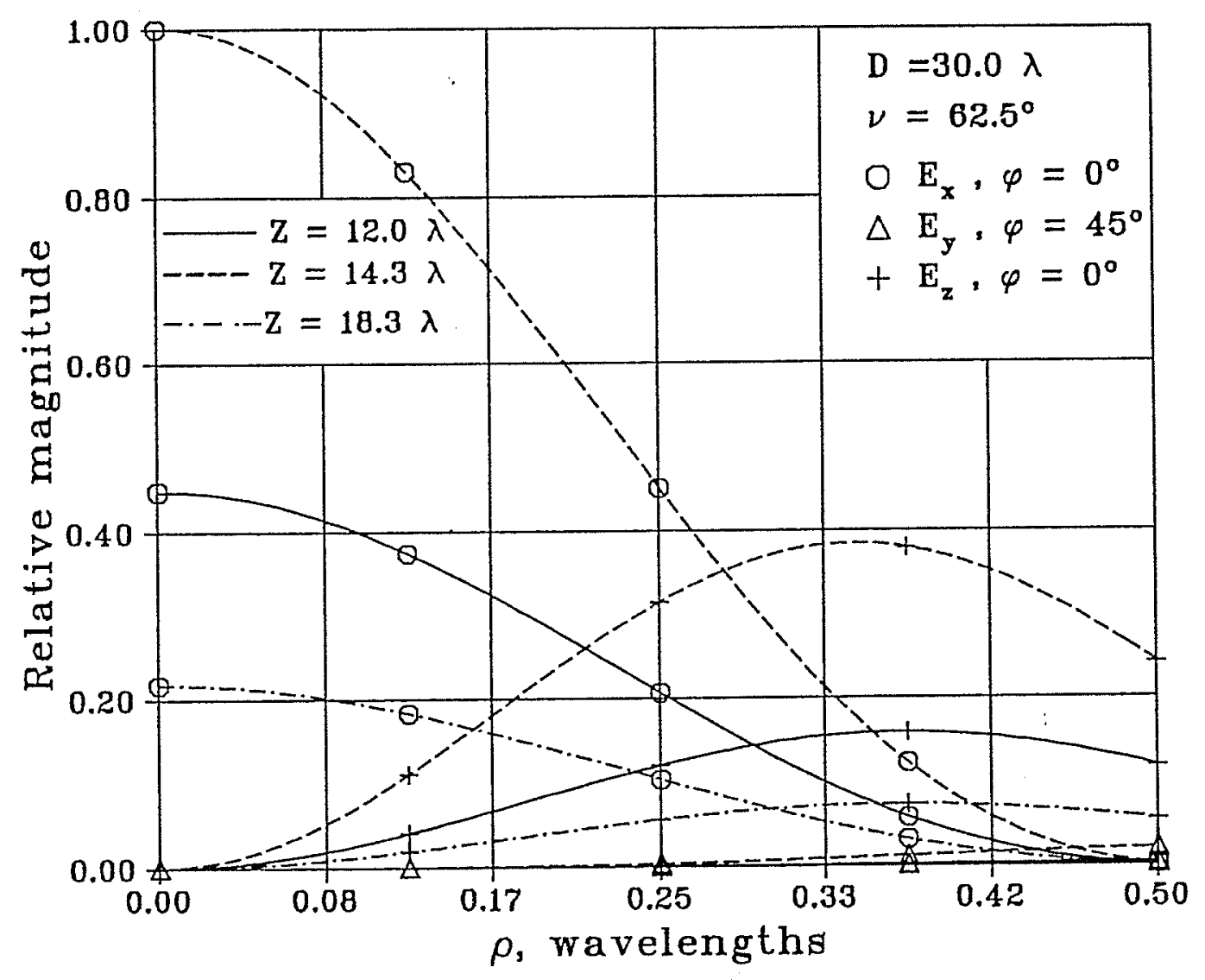

Fig. 2.12 : Variation of focal region field distributionf as a function of radial distance. 


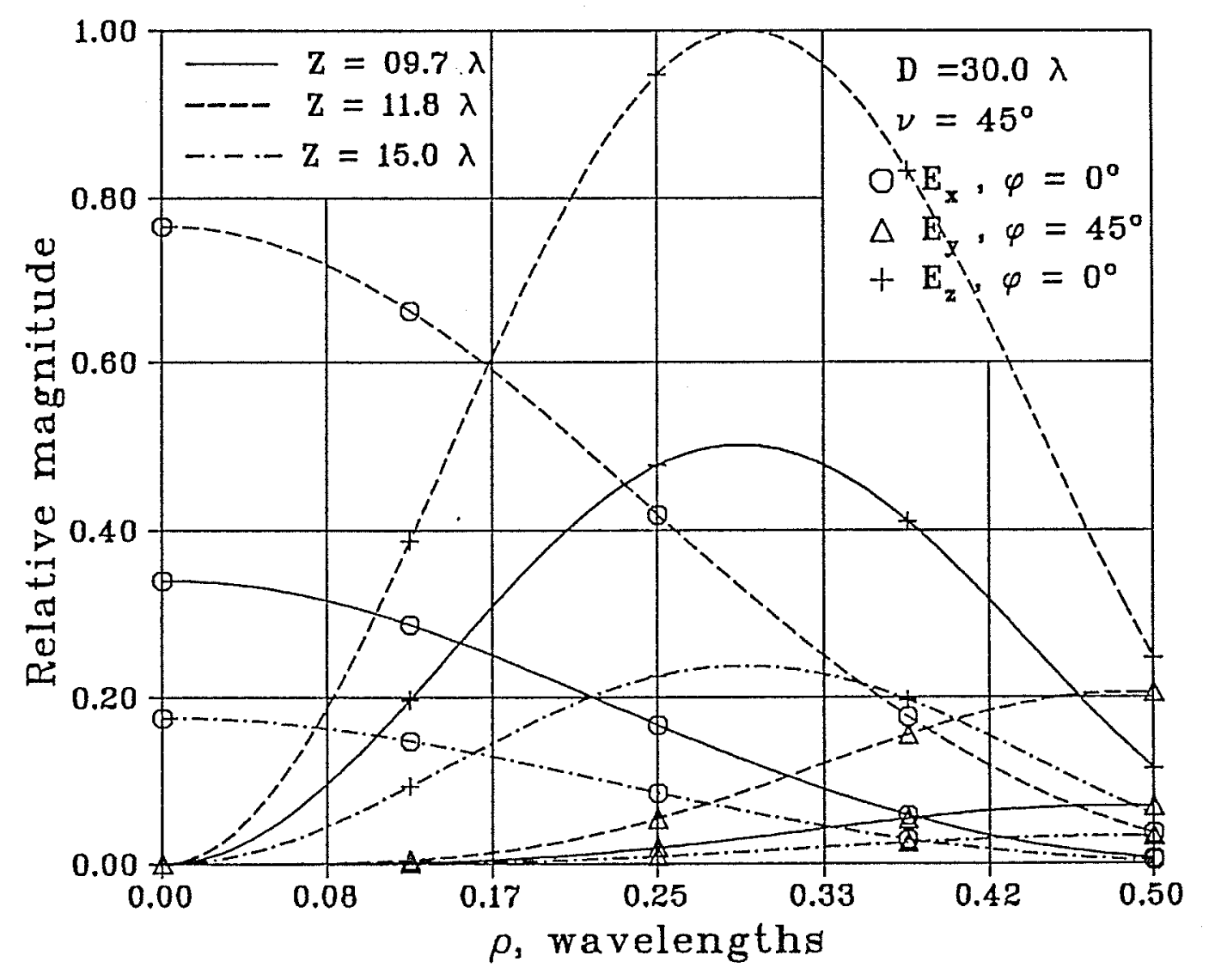

Fig. 2.13 : Variation of focal region field distributions as a function of radial distance. 


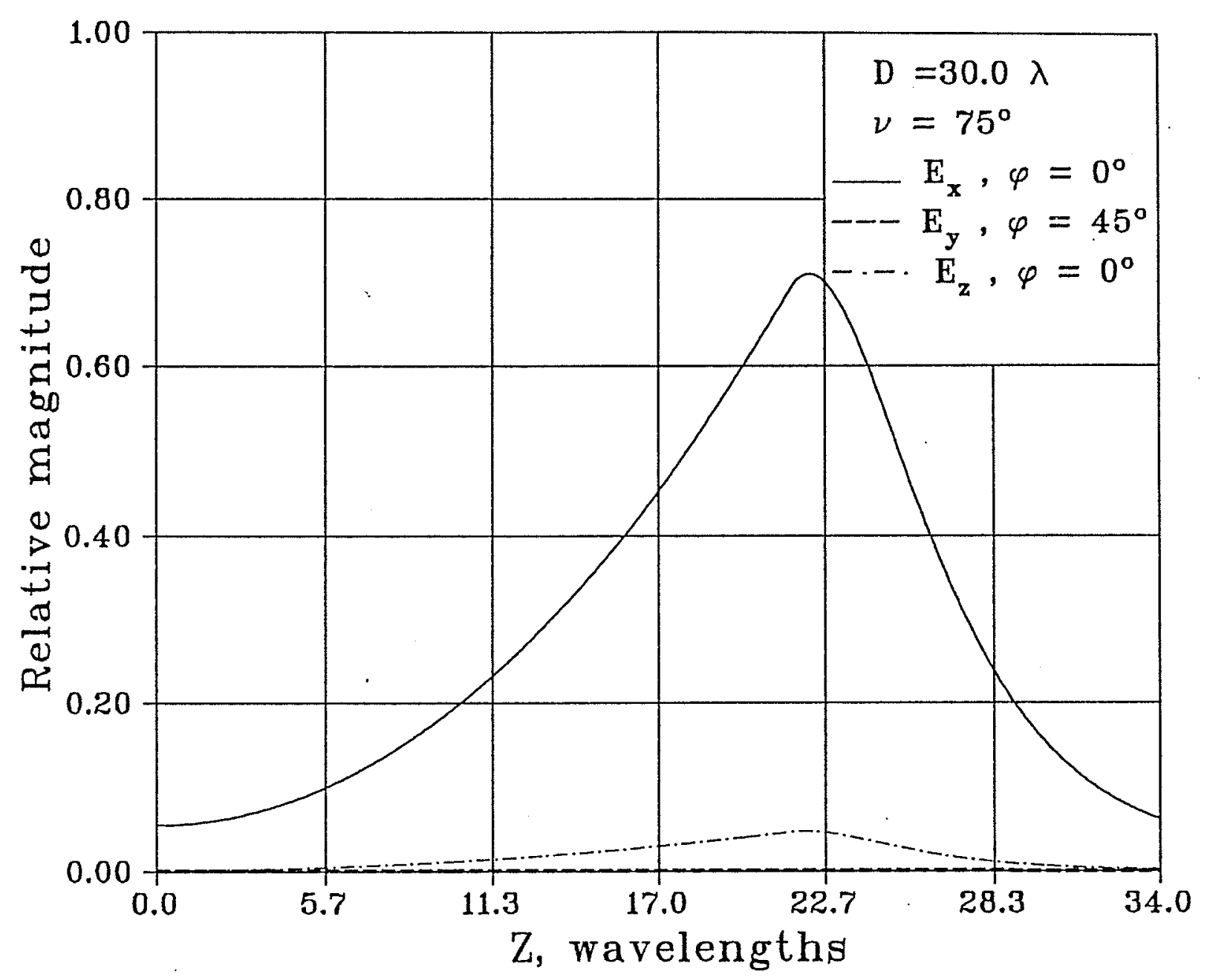

Fig. 2.14 : Hypothetical focal field distributions on a cylindrical surface of $\rho=0.25 \lambda$, generated by curve fittings for data of Fig. 2.5. 


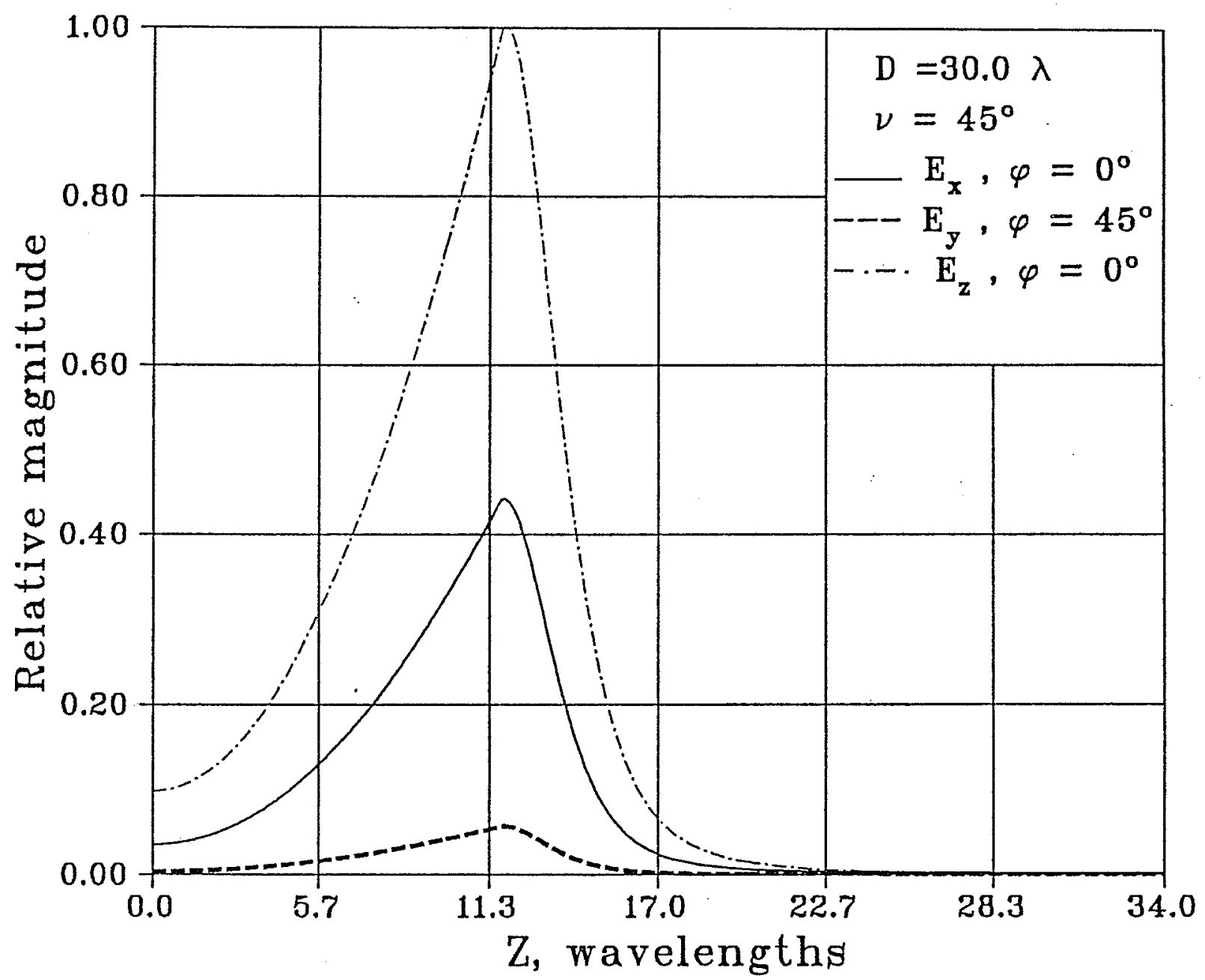

Fig. 2.15 : Hypothetical focal field distributions on a cylindrical surface of $\rho=0.25 \lambda$, generated by curve fittings for data of Fig. 2.7. 


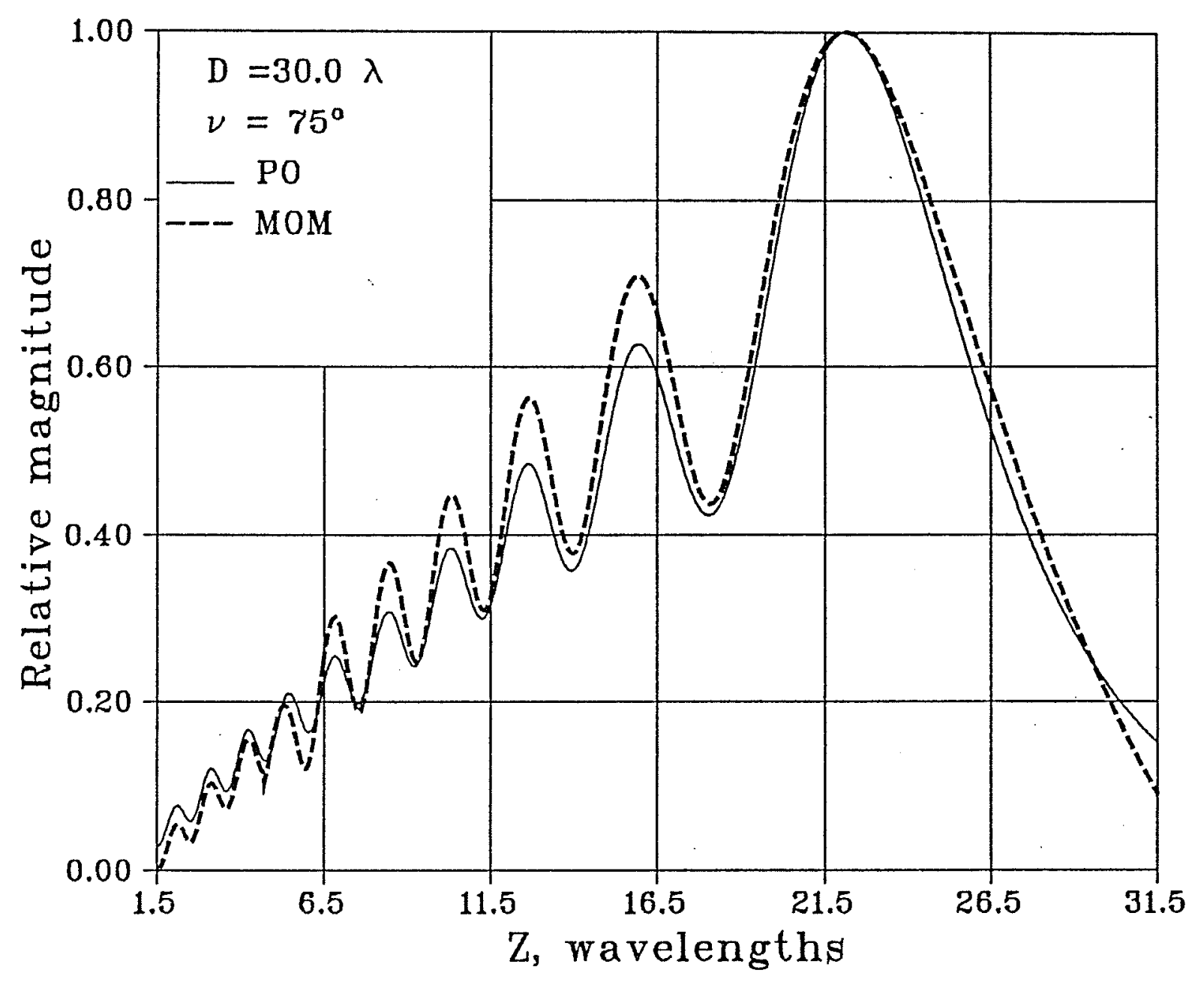

Fig. 2.16 : Calculated co-polar field component $\mathrm{E}_{\mathrm{x}}$ on the focal line, $\varphi=0^{0}$, by the present work (PO) and the method of moments (MOM). 


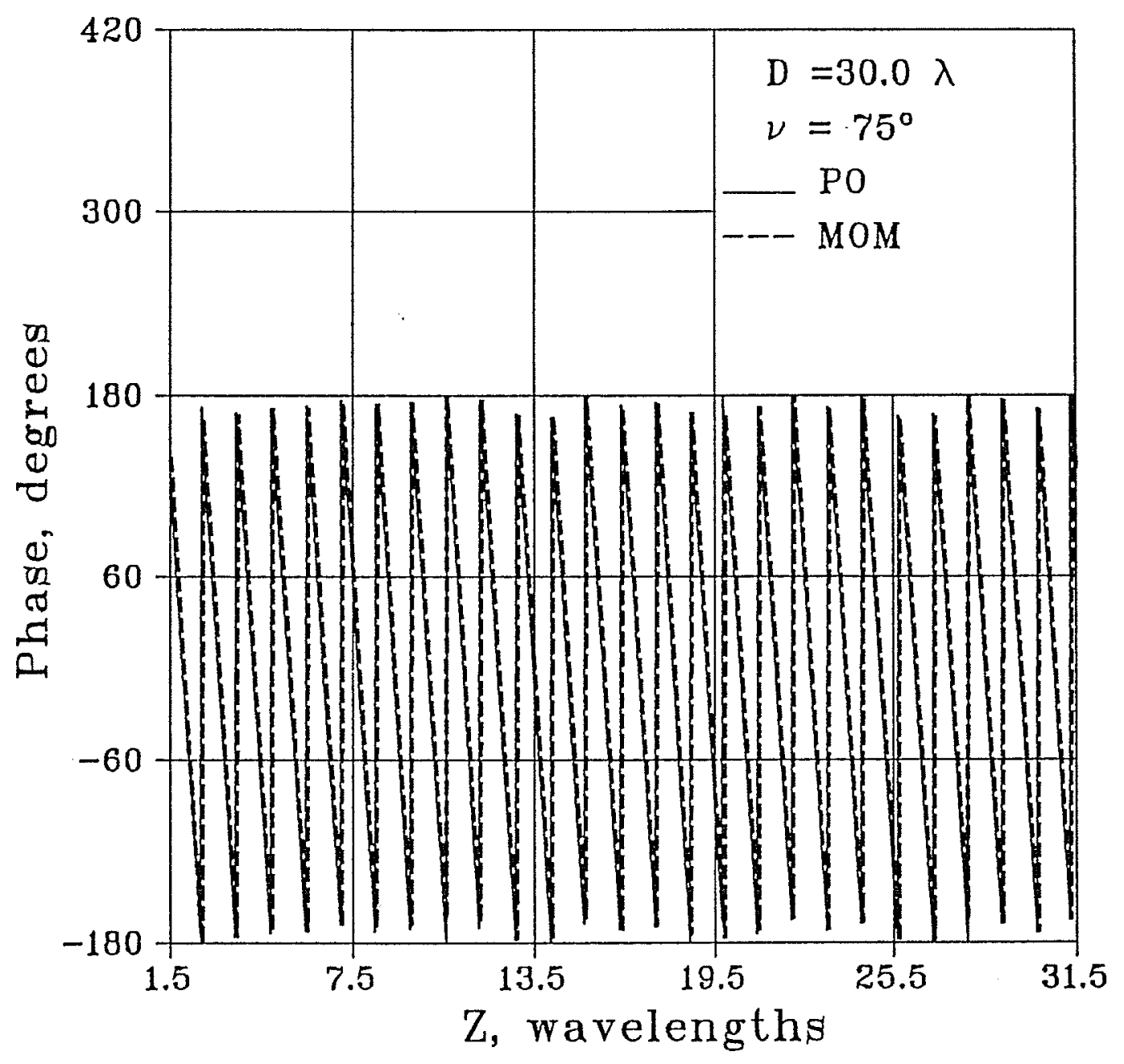

Fig. 2.17: Phase distributions for the calculated co-polar field component of Fig. 2.16. 


\section{CHAPTER III}

\section{Radiation from Conical Reflectors \\ With Dipole Arrays}

\subsection{Introduction}

The investigation of the focal region field in the previous chapter shows that for large angle cones, both cross-polar and axial components are negligibly small near the axis. Thus, for antennas with a moderate cross-polar radiation, a linearly polarized array, such as a dipole array may be used as their line source. On the other hand for a $90^{\circ}$ cone, the axial component is also significant in magnitude near the axis, and a dual polarized array must be used. Using dipoles, such an array may be simulated by two separate arrays, one polarized along the co-polar field and the other along the cone axis. These arrays are selected because of the fact that, line sources using slotted waveguides have already been studied [6,44], and dipole arrays can be designed and fabricated readily using the microstrip technology.

In this work we have used the focal region field to determine the excitation of each dipole, i.e. array elements, at their location. The selected array excitations are then used to compute the line source field and subsequently the scattered field of the reflector, to study the reflector performance.

In the following sections, the expressions for the radiation patterns of conical reflectors with dipole arrays as feeds, are derived and used to compute the radiation patterns. For selected cases numerical data for both co- and cross-polar patterns are generated to examine the conical reflector performance with these simple feeds. 


\subsection{Wide Angle Cone with Linearly Polarized Feeds}

Consider a line source feed in the form of an array of linear dipoles oriented parallel to the $\mathrm{x}$-axis and located along the z-axis of the coordinate system of Fig. 3.1. For a perfectly conducting cone, the far zone field radiated by the induced current distribution $\mathbf{J}$ may be computed from [51] :

$$
\mathbf{E}^{s}=\frac{-j k Z_{0}}{4 \pi R} e^{-j k R} \int_{s}\left[\mathbf{J}-\left(\mathbf{J} \cdot \hat{a}_{R}\right) \hat{a}_{R}\right] e^{j k \mathbf{r}^{\prime} \cdot \hat{a}_{R}} d s^{\prime}
$$

The incident magnetic field from the feed (Appendix A ) on the conical reflector, can be written in the rectangular coordinates as :

$$
\mathbf{H}_{\text {Feed }}^{i n c}=\frac{j}{4 \pi} \sum_{i=1}^{N_{s c}} B_{s c i}\left[-\left(\rho^{\prime} \cot v-d_{s c i}\right) \hat{a}_{y}+\left[\rho^{\prime} \sin \phi^{\prime}\right] \hat{a}_{z}\right]
$$

in which ;

$$
\begin{aligned}
& B_{s c i}=\frac{I_{s c}(i) e^{-j k d_{s c i} \cos \theta_{o}}}{\left[\left(\rho^{\prime} \sin \phi^{\prime}\right]^{2}+\left[\rho^{\prime} \cot v-d_{s c i}\right]^{2}\right]^{W_{s c i}}} \\
& W_{s c i}=\left[e^{-j k r_{s u i}}-2 \cos \left(\frac{k L_{x}}{2}\right) e^{-j k r_{s c i}}+e^{-j k r_{s i i}}\right] \\
& r_{s u i}=\left[\left(\rho^{\prime} \cos \phi^{\prime}-\frac{L_{x}}{2}\right]^{2}+\left[\rho^{\prime} \sin \phi^{\prime}\right]^{2}+\left[\rho^{\prime} \cot v-d_{s c i}\right)^{2}\right]^{1 / 2} \\
& r_{s c i}=\left[\left(\rho^{\prime} \cos \phi^{\prime}\right]^{2}+\left[\rho^{\prime} \sin \phi^{\prime}\right]^{2}+\left[\rho^{\prime} \cot v-d_{s c i}\right]^{2}\right]^{1 / 2} \\
& r_{s l i}=\left[\left[\rho^{\prime} \cos \phi^{\prime}+\frac{L_{x}}{2}\right]^{2}+\left(\rho^{\prime} \sin \phi^{\prime}\right]^{2}+\left(\rho^{\prime} \cot v-d_{s c i}\right]^{2}\right]^{1 / 2}
\end{aligned}
$$

and $\theta_{o}$ is the direction of the array beam, which is equal to the contained cone angle $2 v$. 


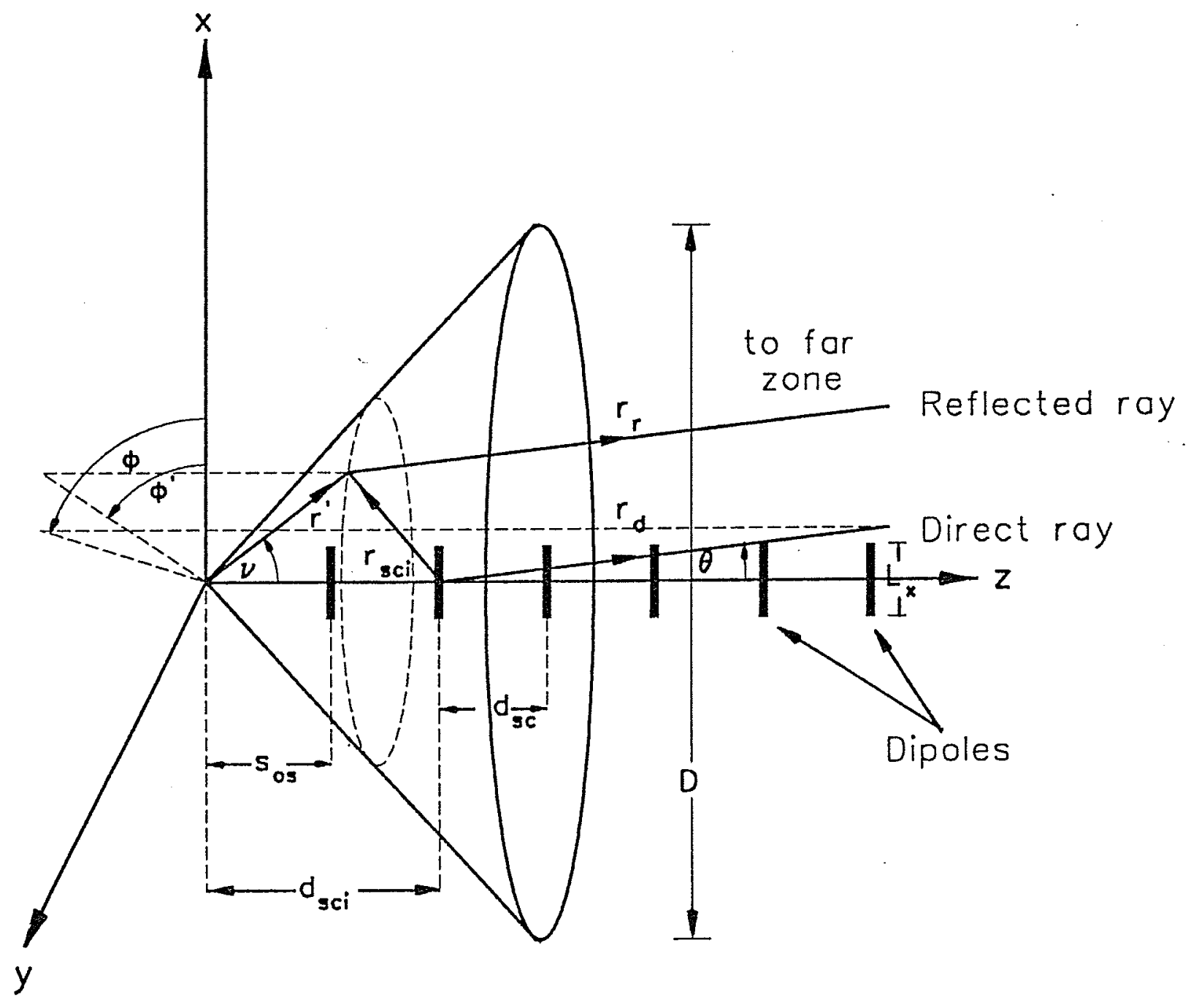

Fig. 3.1: Conical reflector having a line source feed in the form of an array of linear dipoles, oriented parallel to the $x$-axis. 
Using (3.2) and (2.6) in (2.5), one obtains the components of the surface current density as :

$$
\begin{aligned}
& J_{x}=\frac{j}{2 \pi} \sin v \sum_{i=1}^{N_{s c}} B_{s c i}\left[\rho^{\prime} \cot v \cos ^{2} \phi^{\prime}-d_{s c i}\right] \\
& J_{y}=\frac{j}{2 \pi} \sin v \sum_{i=1}^{N_{s c}} B_{s c i}\left[\rho^{\prime} \cot v \sin \phi^{\prime} \cos \phi^{\prime}\right] \\
& J_{z}=\frac{j}{2 \pi} \sin v \sum_{i=1}^{N_{s c}} B_{s c i}\left[\rho^{\prime} \cot ^{2} v \cos \phi^{\prime}-\cot v \cos \phi^{\prime} d_{s c i}\right]
\end{aligned}
$$

Substituting (3.6) in (3.1), one can write the contribution of the rays reflected from the reflector in the far-zone as :

$$
\begin{array}{r}
\mathbf{E}_{r e f}=\frac{-j k Z_{0}}{4 \pi R} e^{-j k R} \int_{0}^{D / 2} \int_{0}^{2 \pi}\left\{\left(\cos \theta \cos \phi J_{x}+\cos \theta \sin \phi J_{y}-\sin \theta J_{z}\right) \hat{a}_{\theta}+\left(-\sin \phi J_{x}+\right.\right. \\
\left.\left.\cos \phi J_{y}\right) \hat{a}_{\phi}\right\} e^{j k \rho^{\prime}\left(\sin \theta \cos \left(\phi-\phi^{\prime}\right)+\cot v \cos \theta\right)} \rho^{\prime} \operatorname{cosec} v d \rho^{\prime} d \phi^{\prime}
\end{array}
$$

and following the same procedure as in [52], the contribution of the direct rays from the feed in the far-zone as ;

$$
\begin{gathered}
\mathbf{E}_{d i r}=\frac{-j k Z_{0}}{4 \pi R} e^{-j k R} \sum_{i=1}^{N_{s c}} I_{s c}(i) e^{j k d_{s i}\left(\cos \theta-\cos \theta_{o}\right)}\left[\frac{\cos \left(\frac{k L_{x}}{2} \sin \theta \cos \phi\right)-\cos \left(\frac{k L_{x}}{2}\right)}{1-\sin ^{2} \theta \cos ^{2} \phi}\right] \\
\cdot\left(\frac{2}{k}\right)\left[\cos \theta \cos \phi \hat{a}_{\theta}-\sin \phi \hat{a}_{\phi}\right]
\end{gathered}
$$

where $\hat{a}_{R}, \hat{a}_{\theta}$ and $\hat{a}_{\phi}$ are unit vectors along a spherical coordinate system $(R, \theta, \phi)$, and ; 
$d_{s c i}=s_{o s}+(i-1) d_{s c}$

$v$ is the cone half angle,

$N_{s c}$ is the number of $\mathrm{x}$-directed dipoles,

$L_{x}$ is the resonant length of the dipole,

$s_{o s}$ is the location of the first dipole from the apex,

$d_{s c}$ is the separation between adjacent dipoles, and,

$I_{s c}(i)$ being the actual or the hypothetical x-component of current according to the focal region distribution.

\subsection{Narrow Angle Cone with Dual Polarized Feed}

Consider a line source feed in the form of two orthogonal arrays of linear dipoles, polarized along $\mathrm{x}$ and $\mathrm{z}$ directions as shown in Fig. 3.2. Following the same procedure as in the previous section, and by making use of Appendices A and B we can write the contribution of the reflected rays from the reflector in the far-zone as :

$$
\begin{gathered}
\mathbf{E}_{r e f}=\frac{-j k Z_{0}}{4 \pi R} e^{-j k R}(j) \int_{0}^{D / 2} \int_{0}^{2 \pi}\left\{\left[E_{\theta u r}+E_{\theta c r}+E_{\theta l r}\right) \hat{a}_{\theta}+\left(E_{\phi u r}+E_{\phi c r}+E_{\phi l r}\right] \hat{a}_{\phi}\right\} \\
e^{j k \rho^{\prime}\left(\sin \theta \cos \left(\phi-\phi^{\prime}\right)+\cot v \cos \theta\right)} \rho^{\prime} d \rho^{\prime} d \phi^{\prime}
\end{gathered}
$$

where ;

$$
\begin{gathered}
E_{\theta u r}=\sum_{i=1}^{N_{z u}} B_{r u i}\left\{\cos \theta \cos \phi\left(-\rho^{\prime} \cos \phi^{\prime}+\frac{L_{w}}{2}\right]+\cos \theta \sin \phi\left(-\rho^{\prime} \sin \phi^{\prime}\right)\right. \\
\left.-\sin \theta\left[-\rho^{\prime} \cot v+\cot v \cos \phi^{\prime} \frac{L_{w}}{2}\right)\right\}
\end{gathered}
$$




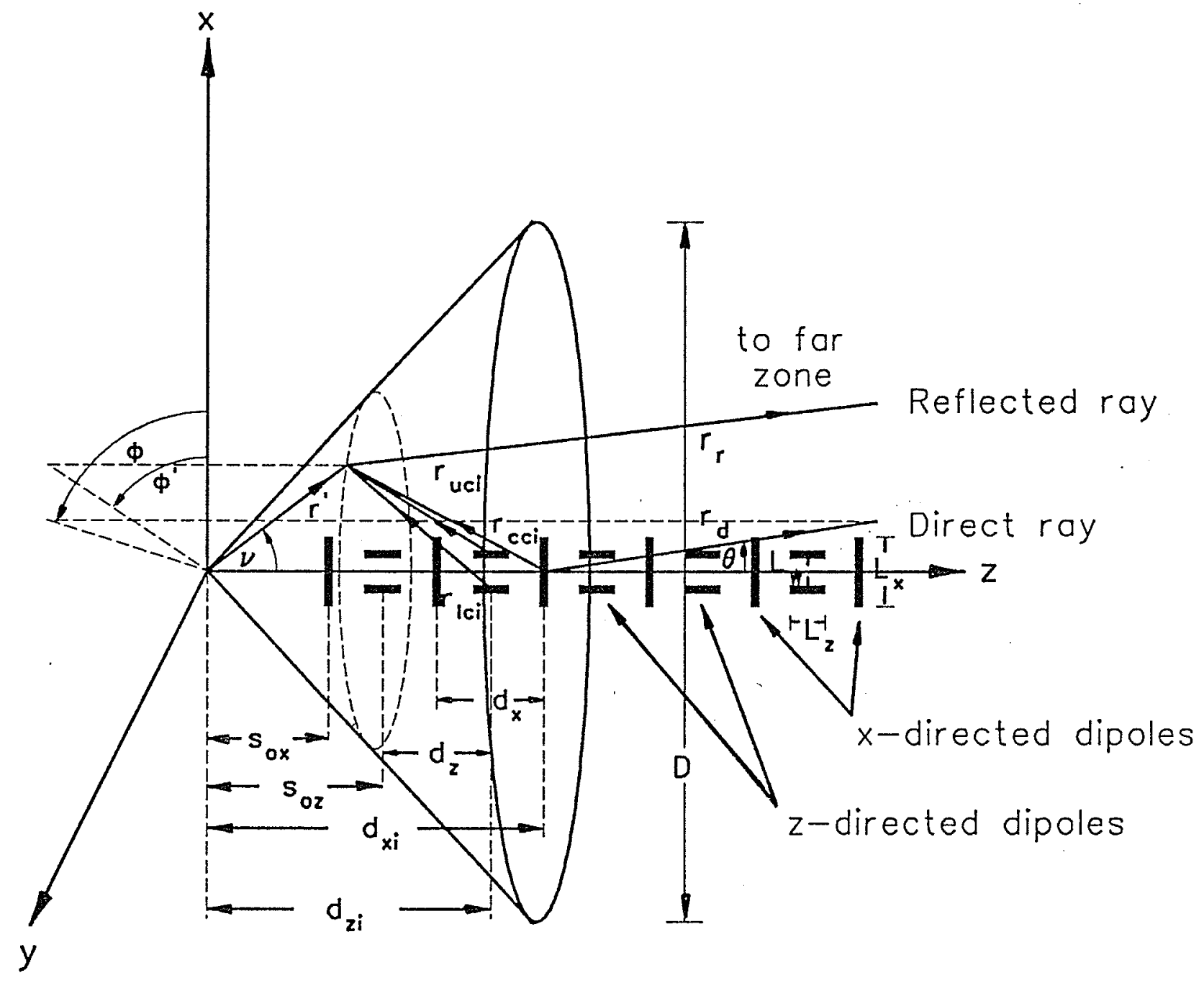

Fig. 3.2 : Conical reflector having a line source feed in the form of two orthogonal arrays of linear dipoles, polarized along $x$ and $z$ directions. 


$$
\begin{aligned}
& E_{\theta c r}=\sum_{i=1}^{N_{x c}} B_{r c i}\left\{\cos \theta \cos \phi\left[\rho^{\prime} \cot v \cos ^{2} \phi^{\prime}-d_{x i}\right]+\cos \theta \sin \phi\left(\rho^{\prime} \cot v \sin \phi^{\prime} \cos \phi^{\prime}\right]\right. \\
& \left.-\sin \theta\left[+\rho^{\prime} \cot ^{2} v \cos \phi^{\prime}-\cot v \cos \phi^{\prime} d_{x i}\right]\right\} \\
& E_{\theta l r}=\sum_{i=1}^{N_{z l}} B_{r l i}\left\{\cos \theta \cos \phi\left(-\rho^{\prime} \cos \phi^{\prime}-\frac{L_{w}}{2}\right)+\cos \theta \sin \phi\left(-\rho^{\prime} \sin \phi^{\prime}\right)\right. \\
& \left.-\sin \theta\left[-\rho^{\prime} \cot v-\cot v \cos \phi^{\prime} \frac{L_{w}}{2}\right]\right\} \\
& E_{\phi u r}=\sum_{i=1}^{N_{z u}} B_{r u i}\left\{-\sin \phi\left(-\rho^{\prime} \cos \phi^{\prime}+\frac{L_{w}}{2}\right)+\cos \phi\left(-\rho^{\prime} \sin \phi^{\prime}\right)\right\} \\
& E_{\phi c r}=\sum_{i=1}^{N_{x c}} B_{r c i}\left\{-\sin \phi\left[\rho^{\prime} \operatorname{cotv} \cos ^{2} \phi^{\prime}-d_{x i}\right]+\right. \\
& \left.\cos \phi\left[\rho^{\prime} \operatorname{cotv} \sin \phi^{\prime} \cos \phi^{\prime}\right]\right\} \\
& E_{\phi l r}=\sum_{i=1}^{N_{z l}} B_{r l i}\left\{-\sin \phi\left(-\rho^{\prime} \cos \phi^{\prime}-\frac{L_{w}}{2}\right)+\cos \phi\left(-\rho^{\prime} \sin \phi^{\prime}\right)\right\}
\end{aligned}
$$

with ;

$$
\left.\left.B_{r u i}=\frac{I_{z u}(i) e^{-j k \phi_{u \mu}}}{\left[\left[\rho^{\prime} \cos \phi^{\prime}-\frac{L_{w}}{2}\right]^{2}+\left[\rho^{\prime} \sin \phi^{\prime}\right) 2\right.}\right] \frac{2}{k}\right] W_{z u i}
$$




$$
\begin{aligned}
& \left.\left.B_{r c i}=\frac{I_{x c}(i) e^{-j k \phi_{x c}}}{\left[\left(\rho^{\prime} \sin \phi^{\prime}\right]^{2}+\left[\rho^{\prime} \cot \nu-d_{x i}\right)^{2}\right.}\right] \frac{2}{k}\right) W_{x c i} \\
& B_{r l i}=\frac{I_{z l}(i) e^{-j k \phi_{z l}}}{\left[\left[\rho^{\prime} \cos \phi^{\prime}+\frac{L_{w}}{2}\right]^{2}+\left[\rho^{\prime} \sin \phi^{\prime}\right]^{2}\right]}\left(\frac{2}{k}\right) W_{z l i} \\
& W_{z u i}=\frac{1}{2}\left(e^{-j k_{\mu u i}}-2 \cos \left(\frac{k L_{z}}{2}\right) e^{-j k r_{u c i}}+e^{-j k r_{u i}}\right) \\
& W_{x c i}=\frac{1}{2}\left(e^{-j k r_{c k i}}-2 \cos \left(\frac{k L_{x}}{2}\right) e^{-j k_{c c i}}+e^{-j k_{c l i}}\right) \\
& W_{z l i}=\frac{1}{2}\left(e^{-j k r_{l u i}}-2 \cos \left(\frac{k L_{z}}{2}\right) e^{-j k r_{l c i}}+e^{-j k r_{l i}}\right)
\end{aligned}
$$

and ;

$$
\begin{aligned}
& r_{u u i}=\left[\left(\rho^{\prime} \cos \phi^{\prime}-\frac{L_{w}}{2}\right)^{2}+\left(\rho^{\prime} \sin \phi^{\prime}\right)^{2}+\left[\rho^{\prime} \cot v-d_{z i}-\frac{L_{z}}{2}\right)^{2}\right]^{1 / 2} \\
& r_{u c i}=\left[\left(\rho^{\prime} \cos \phi^{\prime}-\frac{L_{w}}{2}\right)^{2}+\left(\rho^{\prime} \sin \phi^{\prime}\right){ }^{2}+\left(\rho^{\prime} \cot v-d_{z i}\right)^{2}\right]^{1 / 2} \\
& r_{u l i}=\left[\left(\rho^{\prime} \cos \phi^{\prime}-\frac{L_{w}}{2}\right]^{2}+\left(\rho^{\prime} \sin \phi^{\prime}\right)^{2}+\left[\rho^{\prime} \cot v-d_{z i}+\frac{L_{z}}{2}\right)^{2}\right]^{1 / 2}
\end{aligned}
$$




$$
\begin{aligned}
& r_{c u i}=\left[\left(\rho^{\prime} \cos \phi^{\prime}-\frac{L_{x}}{2}\right]^{2}+\left(\rho^{\prime} \sin \phi^{\prime}\right]^{2}+\left[\rho^{\prime} \cot v-d_{x i}\right]^{2}\right]^{1 / 2} \\
& r_{c c i}=\left[\left(\rho^{\prime} \cos \phi^{\prime}\right)^{2}+\left[\rho^{\prime} \sin \phi^{\prime}\right]^{2}+\left[\rho^{\prime} \cot v-d_{x i}\right]^{2}\right]^{1 / 2} \\
& r_{c l i}=\left[\left(\rho^{\prime} \cos \phi^{\prime}+\frac{L_{x}}{2}\right]^{2}+\left(\rho^{\prime} \sin \phi^{\prime}\right]^{2}+\left(\rho^{\prime} \cot v-d_{x i}\right)^{2}\right]^{1 / 2} \\
& r_{l u i}=\left[\left(\rho^{\prime} \cos \phi^{\prime}+\frac{L_{w}}{2}\right]^{2}+\left[\rho^{\prime} \sin \phi^{\prime}\right]^{2}+\left[\rho^{\prime} \cot v-d_{z i}-\frac{L_{z}}{2}\right]^{2}\right]^{1 / 2} \\
& r_{l c i}=\left[\left[\rho^{\prime} \cos \phi^{\prime}+\frac{L_{w}}{2}\right]^{2}+\left[\rho^{\prime} \sin \phi^{\prime}\right]^{2}+\left[\rho^{\prime} \cot v-d_{z i}\right]^{2}\right]^{1 / 2} \\
& r_{l l i}=\left[\left[\rho^{\prime} \cos \phi^{\prime}+\frac{L_{w}}{2}\right]^{2}+\left(\rho^{\prime} \sin \phi^{\prime}\right]^{2}+\left[\rho^{\prime} \cot v-d_{z i}+\frac{L_{z}}{2}\right]^{2}\right]^{1 / 2}
\end{aligned}
$$

The contribution of the direct rays from the feed in the far-zone can be written as :

$$
\mathbf{E}_{d i r}=\frac{-j k Z_{0}}{4 \pi R} e^{-j k R}\left\{\left(E_{\theta u d}+E_{\theta c d}+E_{\theta l d}\right) \hat{a}_{\theta}+E_{\phi c d} \hat{a}_{\phi}\right\}
$$

where ;

$$
\begin{aligned}
E_{\theta u d}= & \sum_{i=1}^{N_{z u}} I_{z u}(i) e^{-j k \phi_{z u}} e^{+j \frac{k L_{x}}{2} \sin \theta \cos \phi} e^{j k d_{z i} \cos \theta} \\
& \cdot\left(\frac{2}{k}\right)\left[\frac{\cos \left(\frac{k L_{z}}{2} \cos \theta\right)-\cos \left(\frac{k L_{z}}{2}\right)}{1-\cos ^{2} \theta}\right](-\sin \theta)
\end{aligned}
$$




$$
\begin{aligned}
& E_{\theta c d}=\sum_{i=1}^{N_{x c}} I_{x c}(i) e^{-j k \phi_{x c}} e^{j k d_{x i} \cos \theta} \\
& \cdot\left(\frac{2}{k}\right]\left[\frac{\cos \left(\frac{k L_{x}}{2} \sin \theta \cos \phi\right)-\cos \left(\frac{k L_{x}}{2}\right)}{1-\sin ^{2} \theta \cos ^{2} \phi}\right](\cos \theta \cos \phi) \\
& E_{\theta l d}=\sum_{i=1}^{N_{z l}} I_{z l}(i) e^{-j k \phi_{z l}} e^{-j \frac{k L_{x}}{2} \sin \theta \cos \phi} e^{j k d_{z i} \cos \theta} \\
& \cdot\left(\frac{2}{k}\right]\left[\frac{\cos \left(\frac{k L_{z}}{2} \cos \theta\right)-\cos \left(\frac{k L_{z}}{2}\right)}{1-\cos ^{2} \theta}\right](-\sin \theta) \\
& E_{\phi c d}=\sum_{i=1}^{N_{x c}} I_{x c}(i) e^{-j k \phi_{x c}} e^{j k d_{x i} \cos \theta} \\
& \text { - }\left(\frac{2}{k}\right]\left[\frac{\cos \left(\frac{k L_{x}}{2} \sin \theta \cos \phi\right)-\cos \left(\frac{k L_{x}}{2}\right)}{1-\sin ^{2} \theta \cos ^{2} \phi}\right](-\sin \phi)
\end{aligned}
$$

in which ;

$$
\begin{aligned}
& d_{x i}=s_{o x}+(i-1) d_{x} \\
& d_{z i}=s_{o z}+(i-1) d_{z}
\end{aligned}
$$

$N_{x c}$ is the number of $\mathrm{x}$-directed dipoles,

$N_{z u}\left(=N_{z l}\right) \quad$ is the number of z-directed dipoles,

$L_{x}$ resonant length of x-directed dipole,

$L_{z} \quad$ resonant z-directed dipole,

$L_{w} \quad$ is the separation between the z-directed dipole and its image, 
$s_{o x}$ is the location of the first x-directed dipole from the apex,

$s_{o z}$ is the location of the first z-directed dipole from the apex,

$d_{x}$ is the separation between adjacent $\mathrm{x}$-directed dipoles,

$d_{z}$ is the separation between adjacent $\mathrm{z}$-directed dipoles,

$I_{x c}(i)$ is the actual or the hypothetical x-component of current according to the focal region field distribution,

$I_{z u}(i)\left(=I_{z l}(i)\right)$ is the actual or the hypothetical z-component of current according to the focal region field distribution,

$\phi_{x c}$ is the phase of the $\mathrm{x}$-component of current according to the focal region field distribution, in the plane $\phi=0^{\circ}$.

$\phi_{z u}$ is the phase of the z-component of current according to the focal region field distribution, in the plane $\phi=0^{\circ}$, and ,

$\phi_{z l}$ is the phase of the z-component of current according to the focal region field distribution, in the plane $\phi=180^{\circ}$.

The antenna gain in the boresight direction $G$, is given by $[9,48,53]$ :

$$
G=\frac{P(\theta, \phi)_{\max }}{\frac{P_{t}}{4 \pi}}=\frac{4 \pi A}{\lambda^{2}} \eta=\left(\frac{\pi D}{\lambda}\right)^{2} \eta
$$

where;

$P(\theta, \phi)$ is the power radiated per unit solid angle in direction $\theta, \phi$,

$P_{t}$ is the total power radiated,

$A$ is the aperture area, $D$ is the aperture diameter,

$\left(\frac{\pi D}{\lambda}\right)^{2}$ is the gain of a uniformly illuminated constant phase aperture and,

$\eta$ is the gain factor.

The radiation characteristics of the conical reflector can now be determined from these expressions. 


\subsection{Numerical Results and Discussions for Far Field Patterns}

In the examples to follow, both wide and narrow angles cones are investigated. In each case an appropriate dipole array is selected and the reflector far field data is computed. The number of elements in each array is selected such that, the array length equals approximately the geometrical optics line source length [54,55]. Figs. 3.3 and 3.4 show the behavior of the co-polar and cross-polar field components for a wide angle reflector with an aperture diameter of $30.0 \lambda$, having different array element excitations. The $E_{\theta}$ component is computed in the $E$-plane, $\left(\phi=0^{\circ}\right)$, while the $E_{\phi}$ component is computed in the $H$-plane, $\left(\phi=90^{\circ}\right)$. The cross-polar component is computed using Ludwig's [56] third definition in the plane, $\phi=45^{\circ}$, where it has maximum distribution. Fig. 3.3 shows the results for an array of half wave dipoles having the current excitation given according to the exact focal region field $\left(I=I_{E . F . R . F}\right.$ ) shown in Fig. 2.5, while Fig. 3.4 shows the results for the same array, but with the current excitation given according to the hypothetical focal region field $\left(I=I_{\text {H.F.R.F }}\right.$ ) shown in Fig. 2.14. From Figs. 3.3 and 3.4 it is clear that the peak cross-polarization is $-32 \mathrm{~dB}$ and the first side lobe level in the $H$-plane is $-13 \mathrm{~dB}$, while in the $E$-plane it is close to $-16 \mathrm{~dB}$. The results show that, the reflector performance is not compromized by selecting the simpler focal region field of Fig. 2.14, instead of the original complex one in Fig. 2.5 .

Figs. 3.5 and 3.6 show the behavior of the co-polar and the cross-polar field components for a $90^{\circ}$ conical reflector, having different array element excitations with an aperture diameter of $30.0 \lambda$, having different array element excitations, for a dual polarized array of half wave dipoles. Fig. 3.5 shows the results for dual polarized array of half wave dipoles having the current excitation given according to the exact focal region field $\left(I=I_{E . F R . F}\right)$ shown in Fig. 2.7, while Fig. 3.6 shows the results for the same array, but with the current excitation given according to the hypothetical focal region field $\left(I=I_{H . F R . F}\right)$ shown in Fig. 2.15. From Figs. 3.5 and 3.6 it is clear that the peak cross-polarization for dual polarized feeds drops to less than $-38 \mathrm{~dB}$, and the first side lobe level in the $E$ and $H$-plane is close to $-12 \mathrm{~dB}$, 
while in the $E$-plane it is close to $-15 \mathrm{~dB}$. Again, the use of simpler line source distribution of Fig. 2.15, has not deteriorated the results significantly [57].

Fig. 3.7 shows the behavior of the co-polar and cross-polar field components for a wide angle reflector with an aperture diameter of $30.0 \lambda$, having the current excitation given according focal region field shown in Fig. 2.16. The results obtained using the present work (PO) is compared with the numerical solution based on the method of moments (MOM) [50].The agreement demonstrated in Fig. 3.7, is found to be excellent.

The variation of the antenna gain with the number of array elements $\left(N_{x c}\right)$, and the optimal location of the first array element from the apex $\left(s_{o x}\right)$ is shown in Fig. 3.8. It is clear that, the antenna gain is increased initially by increasing the number of array elements until it reaches maximum and drops thereafter. Also by increasing the number of array elements, to reach the maximum gain, the optimal location of the first array element from the apex is decreased.

In these examples, the use of dipole arrays provides far field patterns with satisfactory gain factors of about $84 \%$ and small cross-polarizations. The computed gain factors include the feed spillover power but exclude the substrate and load losses. They compare favorably with theoretical gain factors of paraboloidal reflectors, normally around $82 \%$. In practice, however, the reflector gain factor will depend on the type of array feed used. With an ideal resonant array the substrate is the only source of loss and when neglected gives the above theoretical gain factor, but within a narrow frequency band. With traveling line arrays, on the other hand, a small percentage of the incident power is lost in the load which reduces the reflector gain factor, but the line source bandwidth is inherently large.

In the above examples the side lobes of the reflector far field patterns are high, and around $-15 \mathrm{~dB}$. This is due to the fact that the focal region fields were determined using a uniform illumination of the reflector aperture by a plane wave. In practice, to reduce the side lobes, one can determine the focal region field using a tapered aperture field [58]. This is clear from both tables 3.1 and 3.2, where the variation of the 
calculated gains, first side lobe levels with the aperture distribution are demonstrated. Table 3.1 shows the variation for the wide angle reflector case, while table 3.2 shows the variation for the narrow angle reflector case. From tables 3.1 and 3.2, we can conclude that the variation of the calculated gains, first side levels with the aperture distribution, are in good agreement with the aperture theory [48].

Figs. $3.9(\mathrm{a} \& \mathrm{~b}), 3.10(\mathrm{a} \& \mathrm{~b})$, and $3.11(\mathrm{a} \& \mathrm{~b})$ show the behavior of the normalized field components, $E_{x}$ and $E_{y}$ over the aperture plane (z=const) of a wide angle conical reflector, at different locations from the apex, using the present work (PO). The current excitation for the feed elements is given according to the exact focal region field $\left(I=I_{E . F . R . F}\right)$ shown in Fig. 2.5.

The above results for both wide and narrow angle cones indicate that dipole arrays can be used as feeds for conical reflectors. Traditionally, line sources were designed using slotted wave guides, which are difficult to design and fabricate. The performance of printed dipoles, on the other hand is easy to understand and techniques for their designs is available [59]. Since their fabrication is relatively simple and low cost, line sources using dipole arrays seem an attractive alternative to slotted wave guides. 


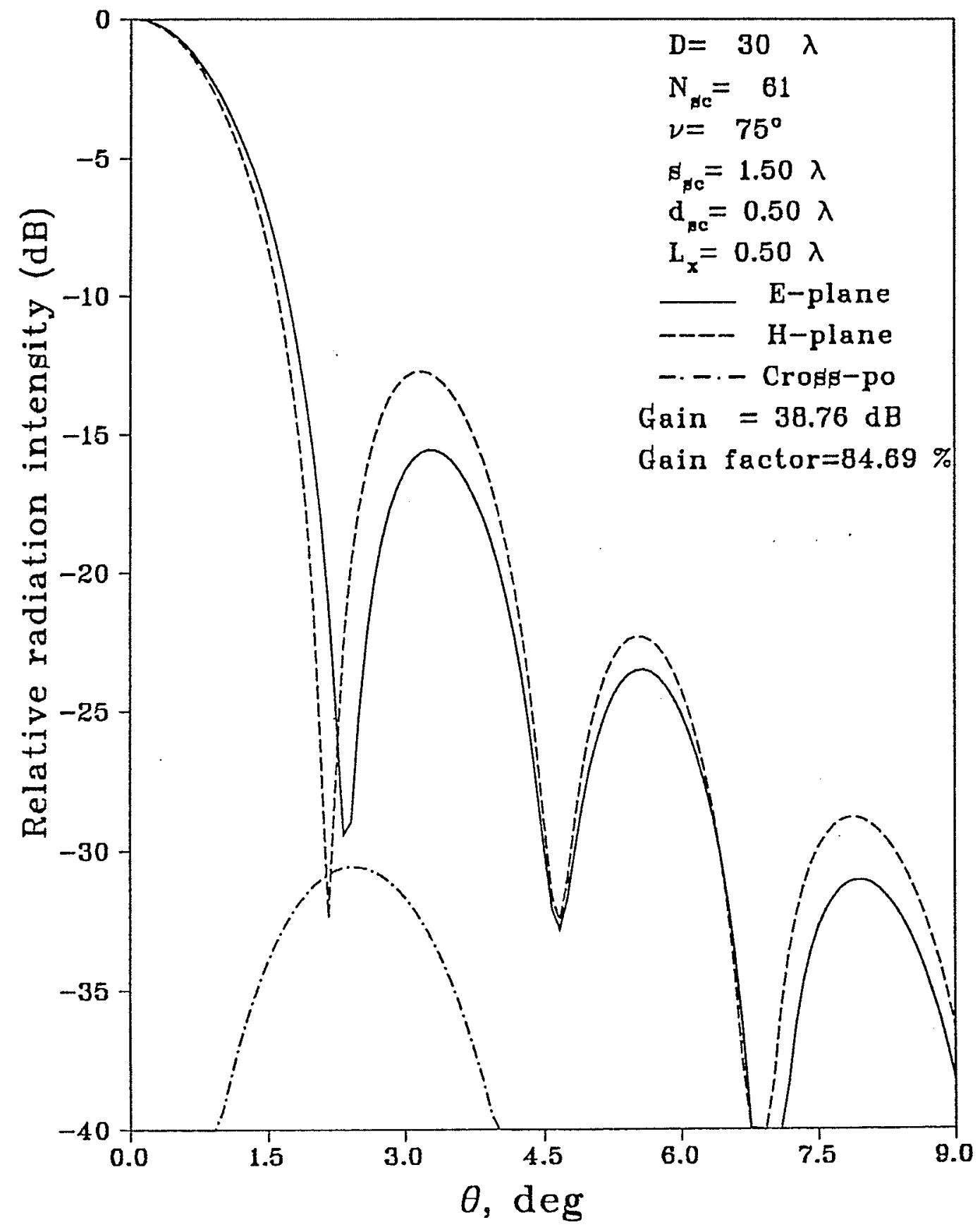

Fig. 3.3 : The radiation pattern 9 of a wide angle conical reflector having a line source feed of linear dipoles oriented parallel to the $\mathrm{x}$-axis, excitation according to focal field distributions of Fig. 2.5. 


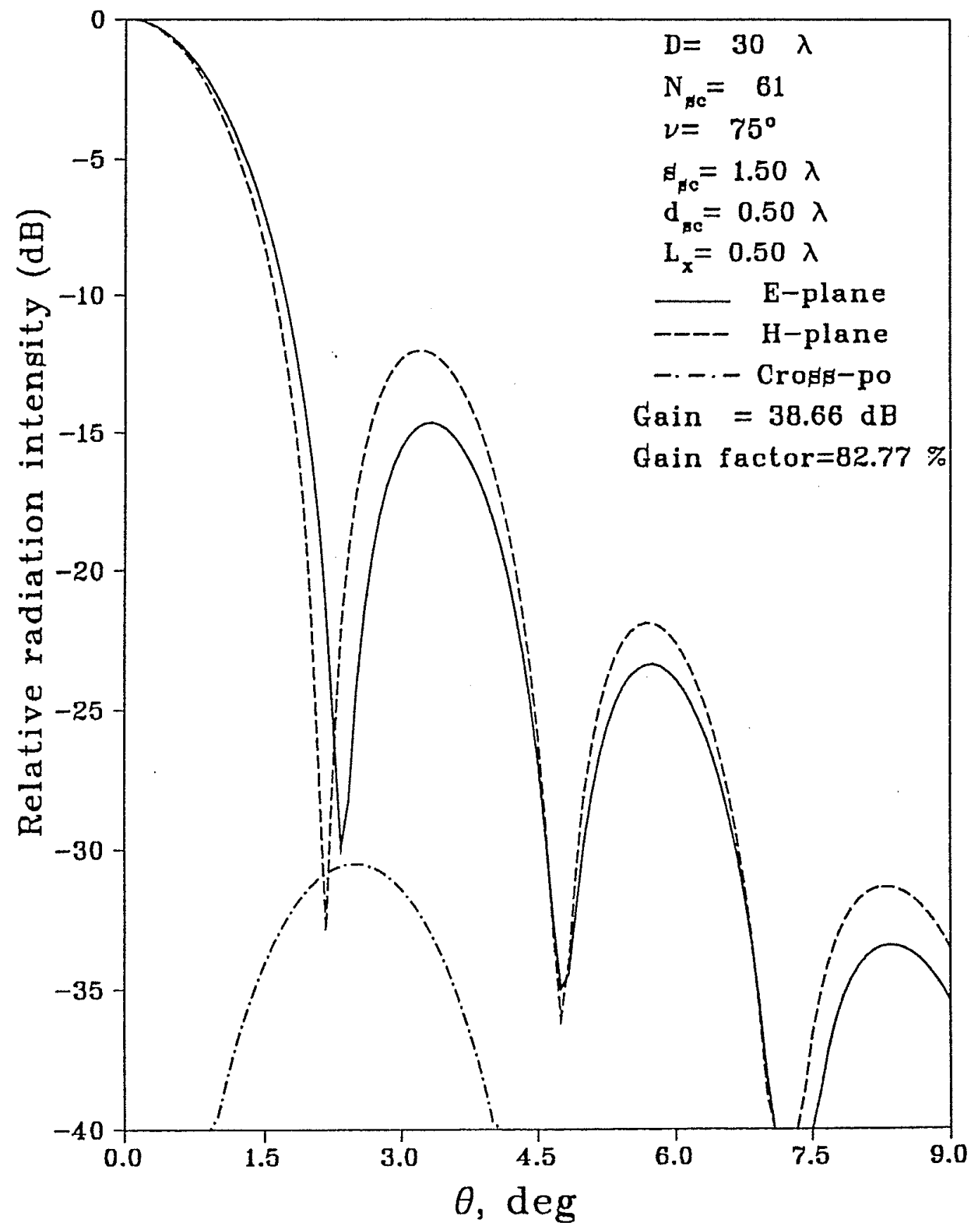

Fig. 3.4 : The radiation patterns of a wide angle conical reflector having a line source feed of linear dipoles oriented parallel to the $\mathrm{x}$-axis, excitation according to a hypothetical focal field diftributions of Fig. 2.14. 


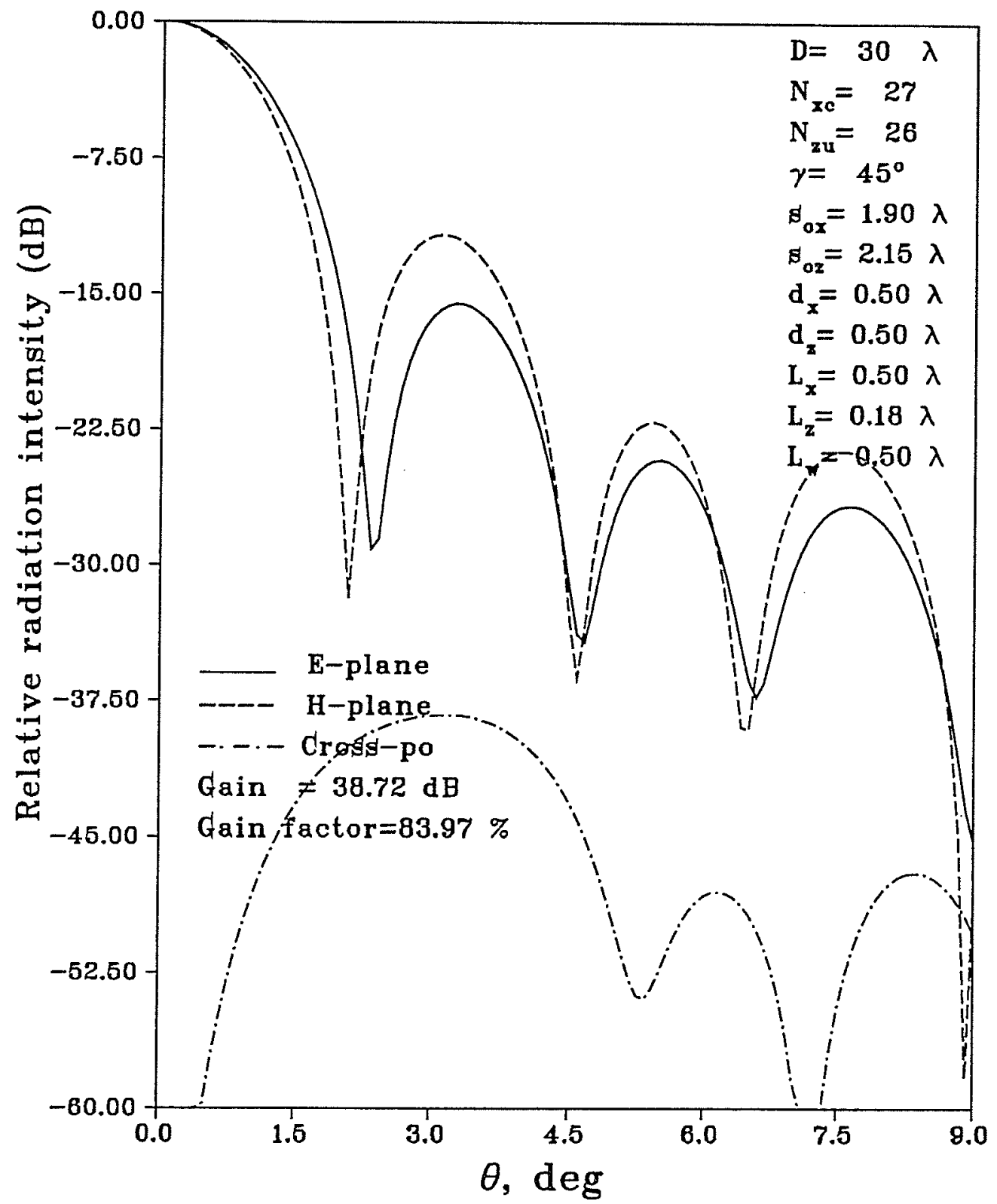

Fig. 3.5 : The radiation patterns of a $90^{\circ}$ conical reflector with a dual polarized line source feed of Fig. 3.2, excitation according to focal field distributions of Fig. 2.7. 


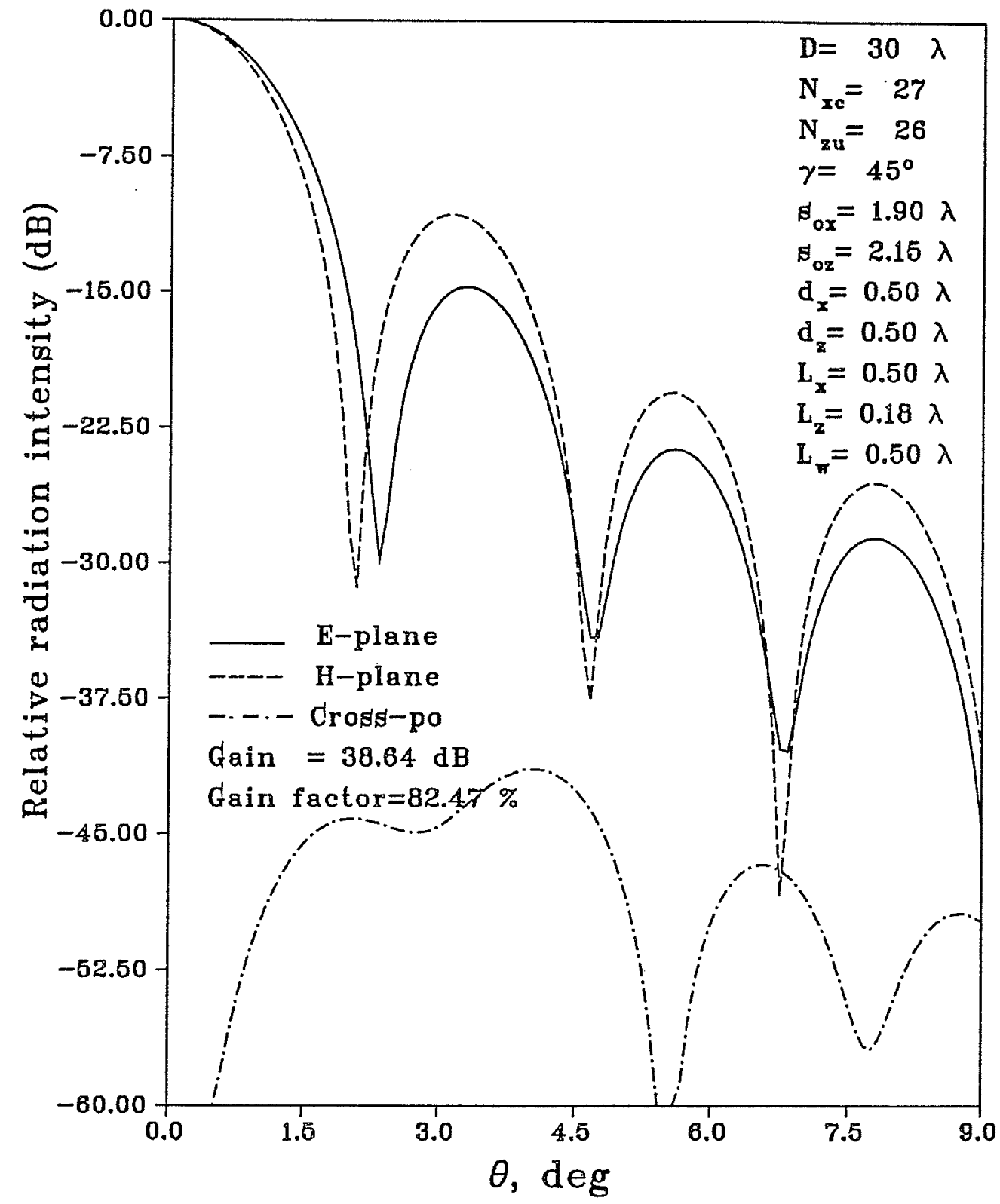

Fig. 3.6 : The radiation patterns of a $90^{\circ}$ conical reflector with a dual polarized line source feed of Fig. 3.2, excitation according to a hypothetical focal field diptributions of Fig. 2.15. 


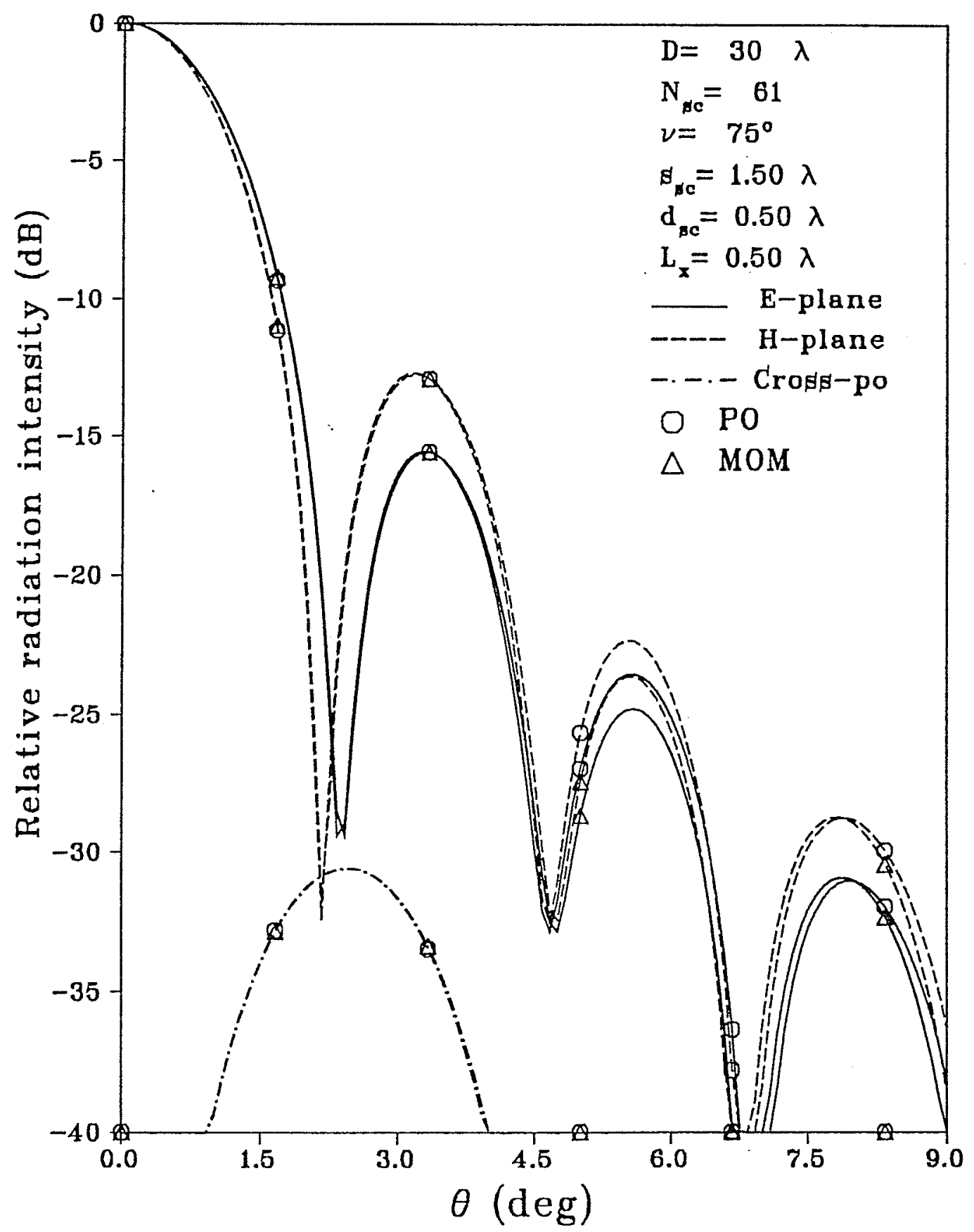

Fig. 3.7 : The radiation pattern $\$$ of a wide angle conical reflector having a line source feed of linear dipoles oriented parallel to the $\mathrm{x}$-axis, excitation according to focal field distribution of Fig. 2.16. 


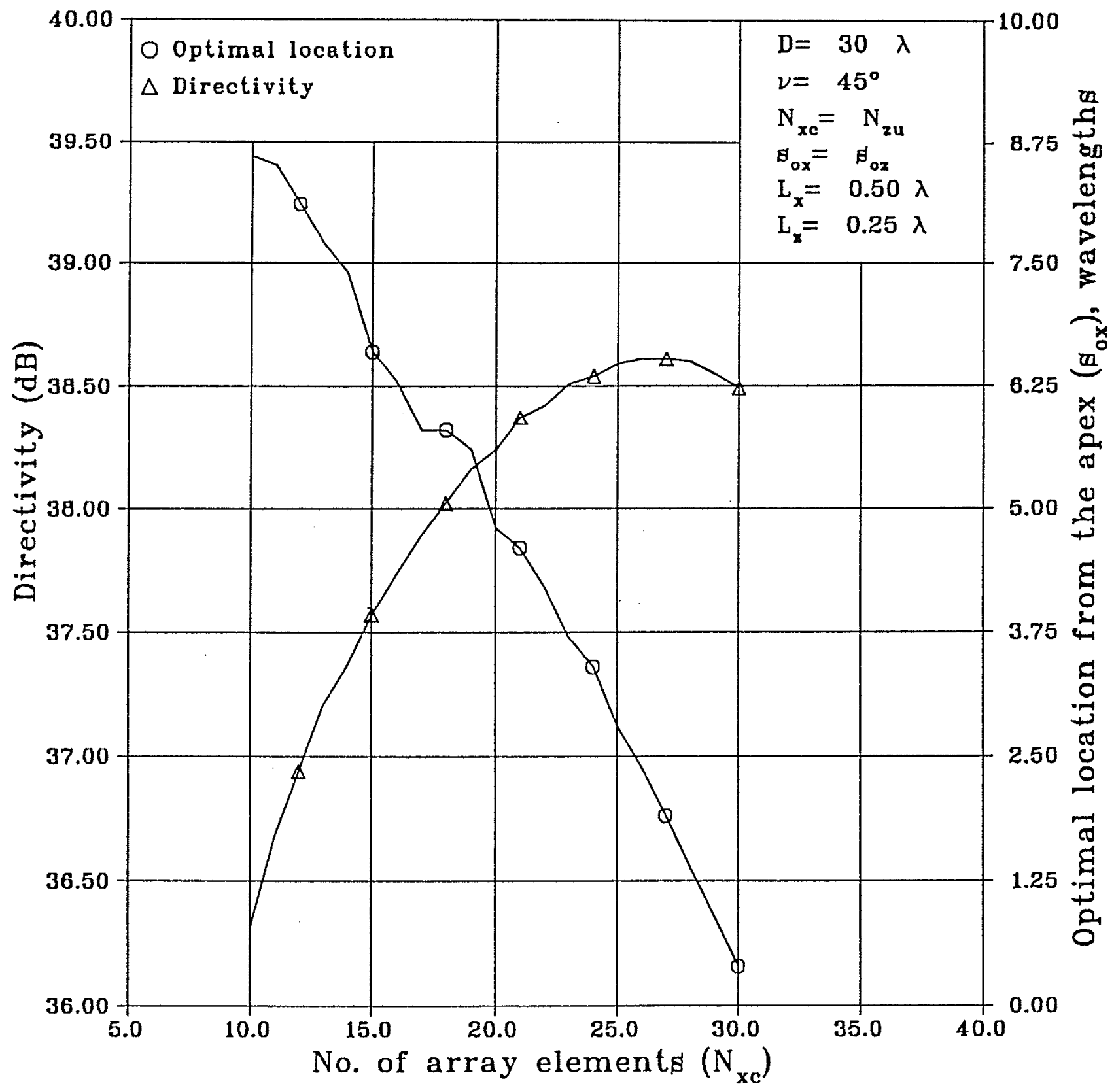

Fig. 3.8 : Variation of antenna gain with the number of array elements $\left(\mathrm{N}_{x c}\right)$, and the optimal location from the apex $\left(\mathrm{s}_{\mathrm{ox}}\right)$. 
Table 3.1

Variation of the calculated gains, first side lobe levels

with the aperture distribution.

$$
\begin{gathered}
\mathrm{D}=30.0 \lambda \quad S_{o s}=1.50 \lambda d_{s c}=0.50 \lambda \\
N_{s c}=61 \quad v=75.0^{\circ}
\end{gathered}
$$

\begin{tabular}{|c|c|c|c|c|}
\hline \multirow{2}{*}{ Aperture distribution } & \multirow{2}{*}{ Gain (dB) } & \multirow{2}{*}{ Gain factor (\%) } & \multicolumn{2}{|c|}{ First side lobe levels (dB) } \\
\cline { 4 - 5 } & & & E-plane & H-plane \\
\hline A & 38.75 & 84.48 & -15.57 & -12.73 \\
B & 37.55 & 64.06 & -22.96 & -18.23 \\
C & 35.91 & 43.98 & -25.91 & -20.49 \\
D & 38.70 & 83.49 & -19.79 & -15.90 \\
E & 38.55 & 80.79 & -20.77 & -16.60 \\
\hline
\end{tabular}

A ...... Uniform plane wave, $\left(1-r^{2}\right)^{0}$

B ...... Taper plane wave, $\left(1-r^{2}\right)^{1}$

C ..... Square taper plane wave, $\left(1-r^{2}\right)^{2}$

$D$...... Taper to 0.75 at the edges plane wave, $0.75+\left(1-r^{2}\right)^{1}$

E ..... Taper to 0.50 at the edges plane wave, $0.50+\left(1-r^{2}\right)^{1}$ 
Table 3.2

Variation of the calculated gains, first side lobe levels

with the aperture distribution.

$$
\begin{gathered}
\mathrm{D}=30.0 \lambda \quad S_{o x}=1.90 \lambda \quad S_{o z}=2.15 \lambda \\
d_{x}=0.50 \lambda \quad d_{z}=0.50 \lambda \\
N_{x c}=27 \quad N_{z u}=26 \quad v=45.0^{\circ}
\end{gathered}
$$

\begin{tabular}{|c|c|c|c|c|}
\hline \multirow{2}{*}{ Aperture distribution } & \multirow{2}{*}{ Gain $(\mathrm{dB})$} & Gain factor $(\%)$ & \multicolumn{2}{|c|}{ First side lobe levels (dB) } \\
\cline { 3 - 5 } & & & E-plane & H-plane \\
\hline A & 38.72 & 83.96 & -15.62 & -11.77 \\
B & 37.46 & 62.73 & -21.62 & -16.10 \\
C & 35.73 & 42.20 & -22.93 & -16.97 \\
D & 38.70 & 83.62 & -19.31 & -14.61 \\
E & 38.55 & 80.79 & -20.11 & -15.16 \\
\hline
\end{tabular}

A ..... Uniform plane wave, $\left(1-r^{2}\right)^{0}$

$B \quad$...... Taper plane wave, $\left(1-r^{2}\right)^{1}$

C ...... Square taper plane wave, $\left(1-r^{2}\right)^{2}$

$D \quad$..... Taper to 0.75 at the edges plane wave, $0.75+\left(1-r^{2}\right)^{1}$

E ..... Taper to 0.50 at the edges plane wave , $0.50+\left(1-r^{2}\right)^{1}$ 


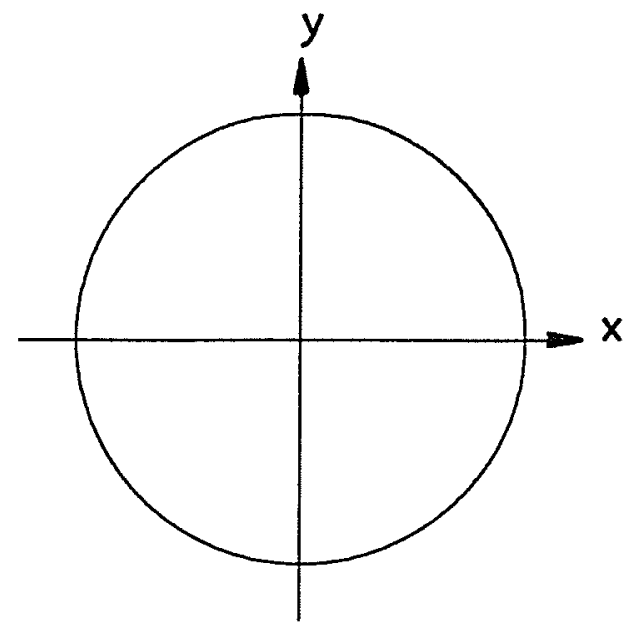

The aperture plane

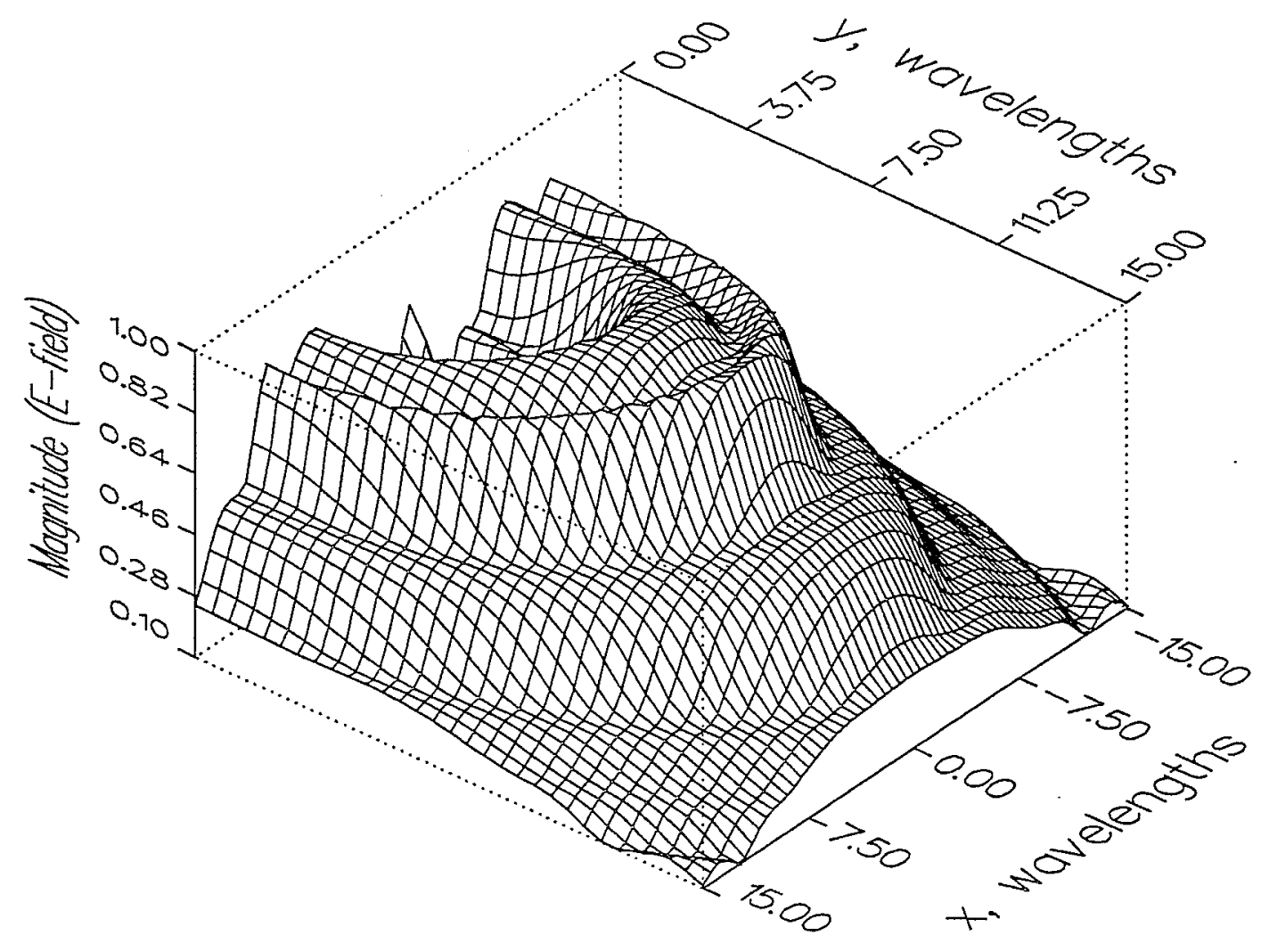

Fig. 3.9a : The field component $\mathrm{E}_{\mathrm{x}}$ over the aperture plane of a wide angle conical reflector $\left(\nu=75^{\circ}\right)$, at a distance $\mathrm{z}=\mathrm{const}=50.0 \lambda$ from the apex. 


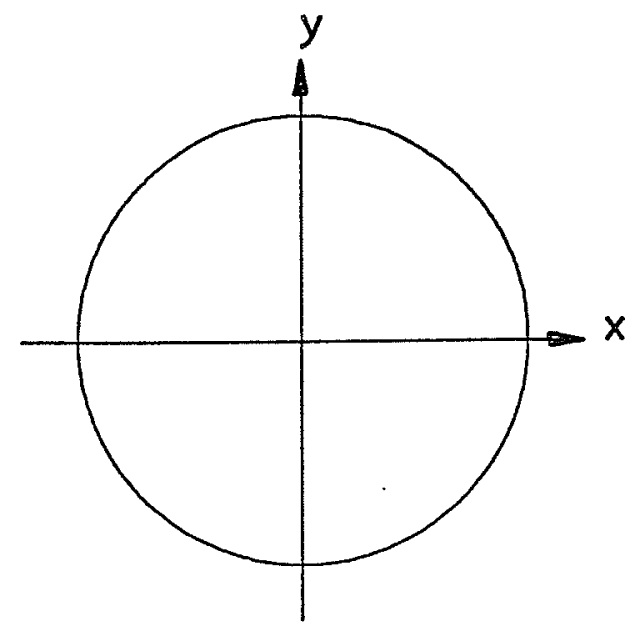

The operture plane

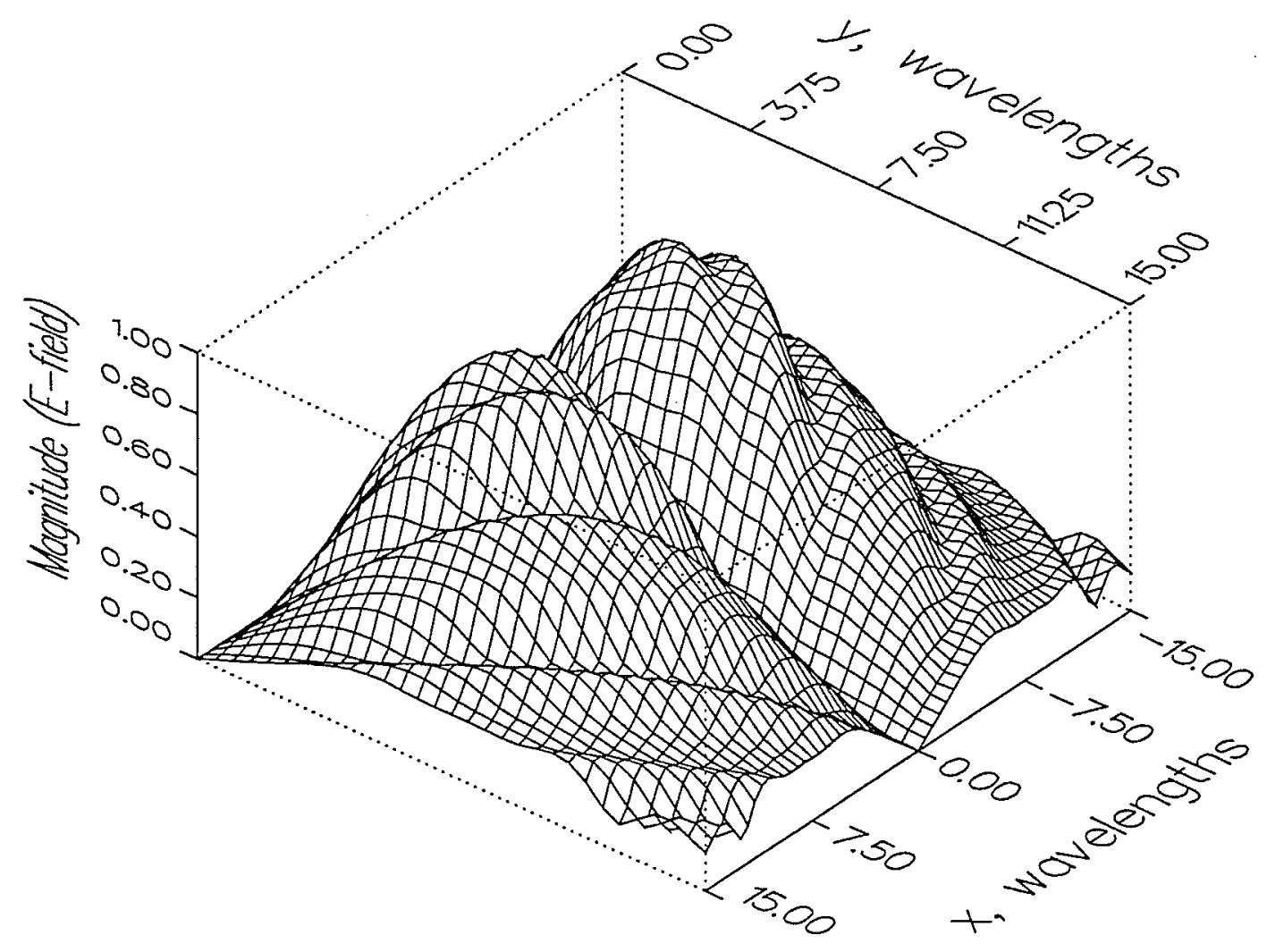

Fig. 3.9b : The field component $\mathrm{E}_{\mathrm{y}}$ over the aperture plane of a wide angle conical reflector $\left(\nu=75^{\circ}\right)$, at a distance $\mathrm{z}=\mathrm{const}=50.0 \lambda$ from the apex. 


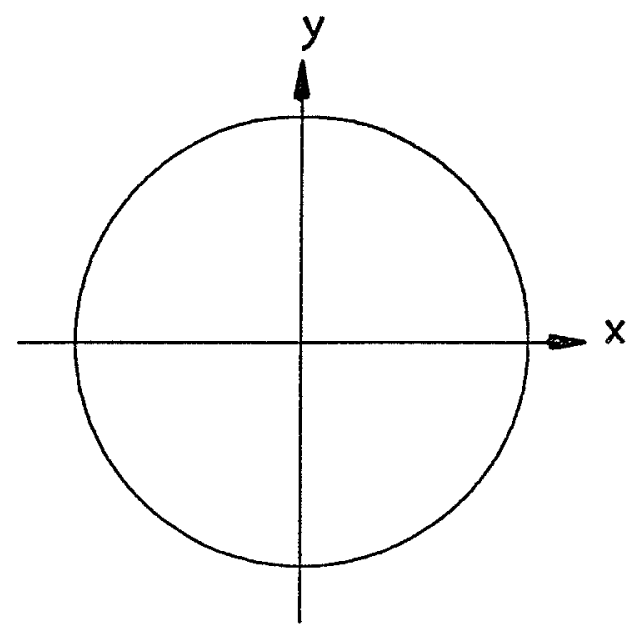

The operture plane

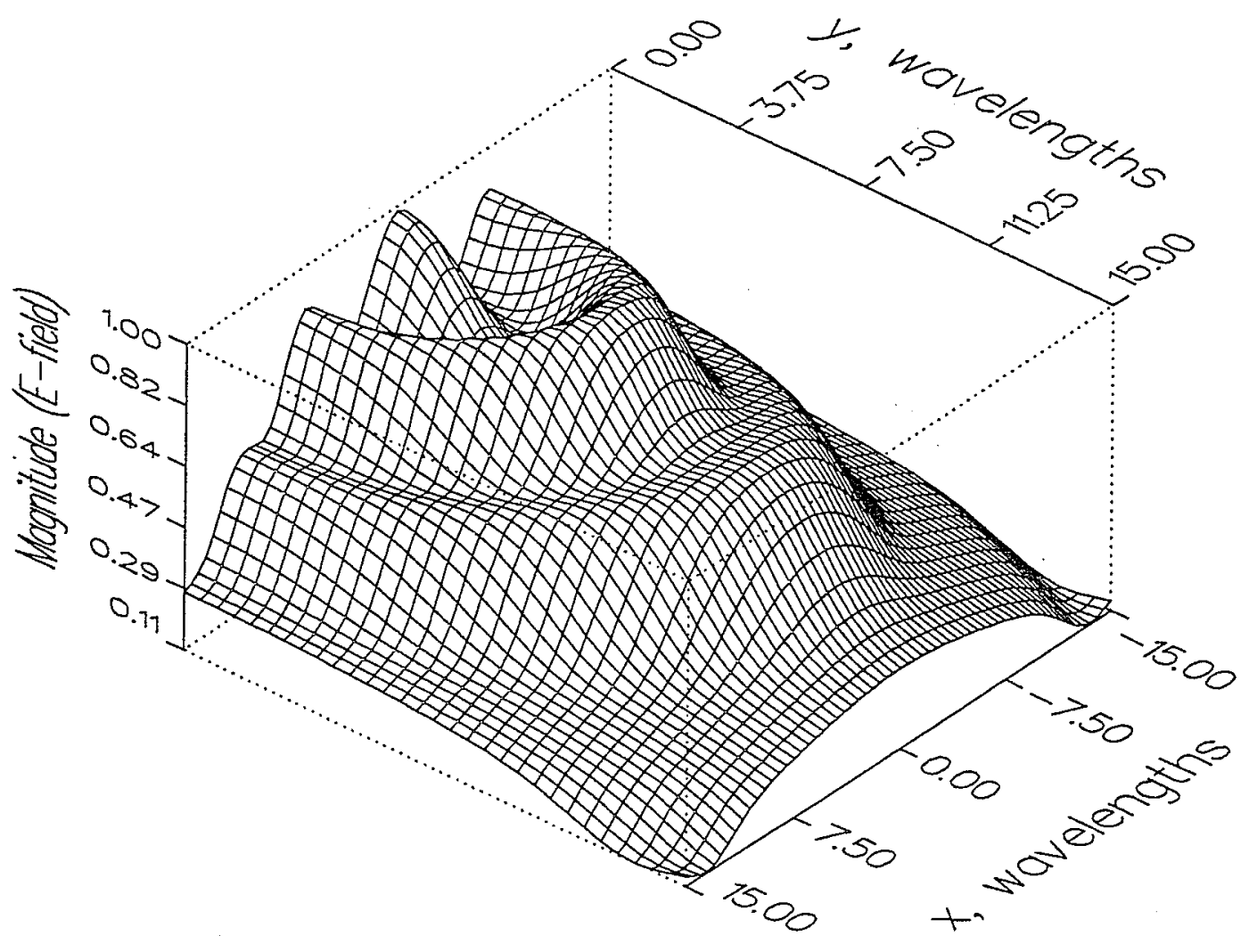

Fig. 3.10a : The field component $E_{x}$ over the aperture plane of a wide angle conical reflector $\left(\nu=75^{\circ}\right)$, at a distance $\mathrm{z}=\mathrm{const}=100.0 \lambda$ from the apex. 


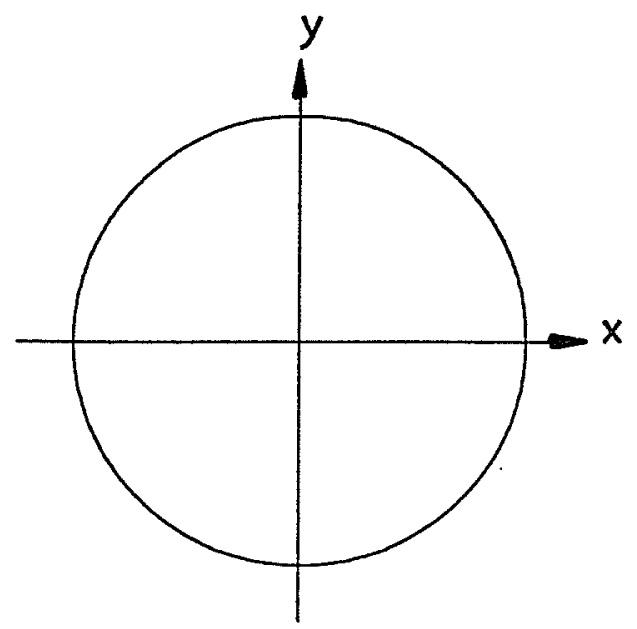

The aperture plane

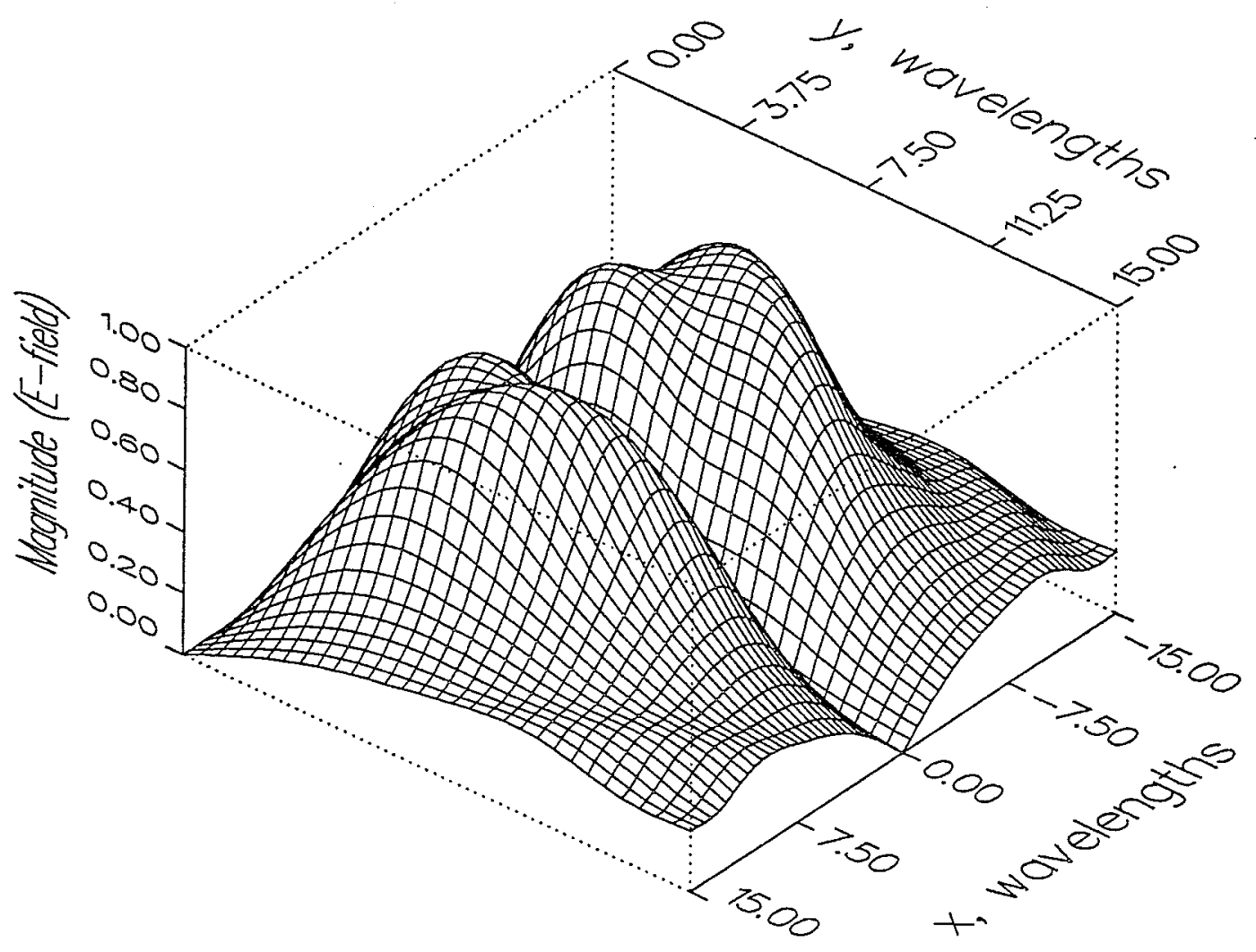

Fig. 3.10b : The field component $E_{y}$ over the aperture plane of a wide angle conical reflector $\left(\nu=75^{\circ}\right)$, at a distance $z=$ const $=100.0 \lambda$ from the apex. 


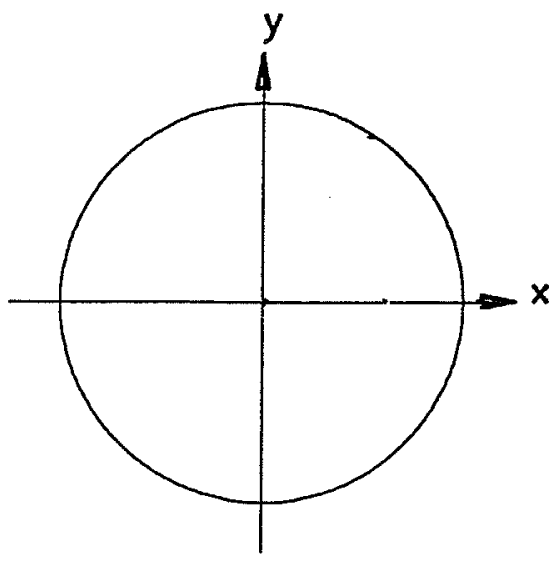

The aperture plane

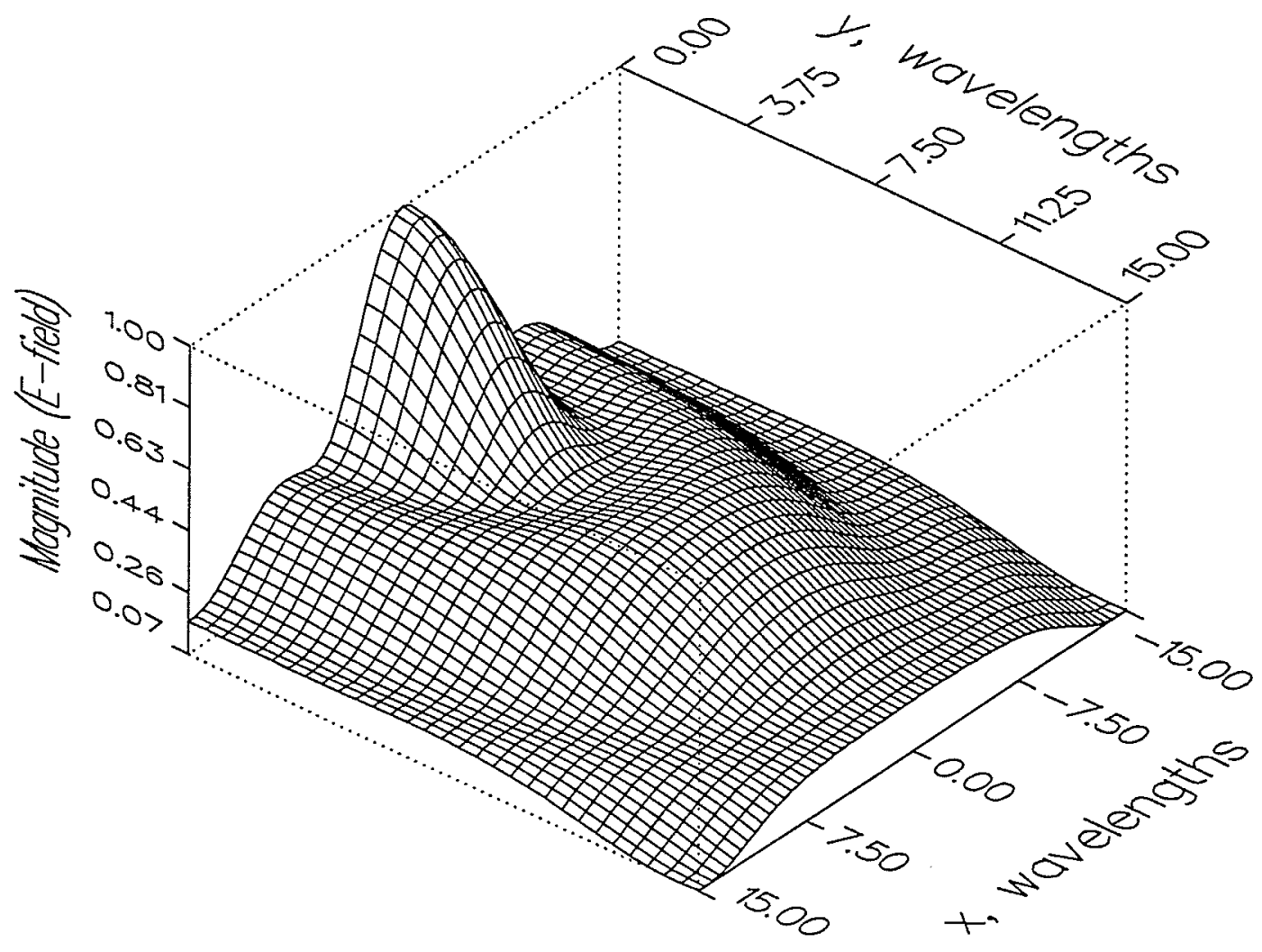

Fig. 3.11a : The field component $E_{x}$ over the aperture plane of a wide angle conical reflector $\left(\nu=75^{\circ}\right)$, at a distance $z=$ const $=150.0 \lambda$ from the apex. 


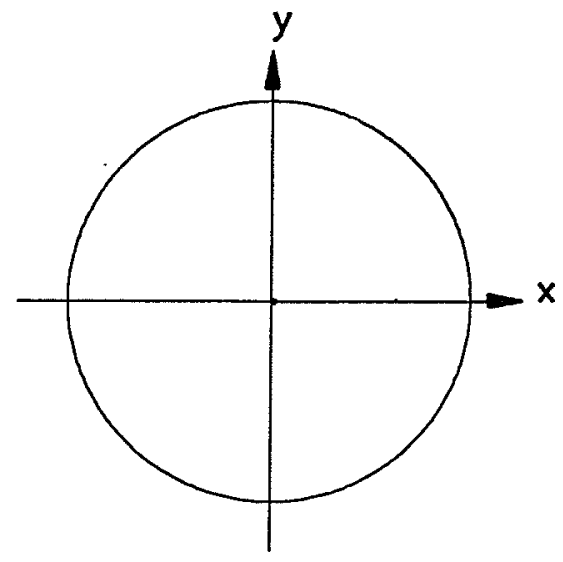

The operture plane

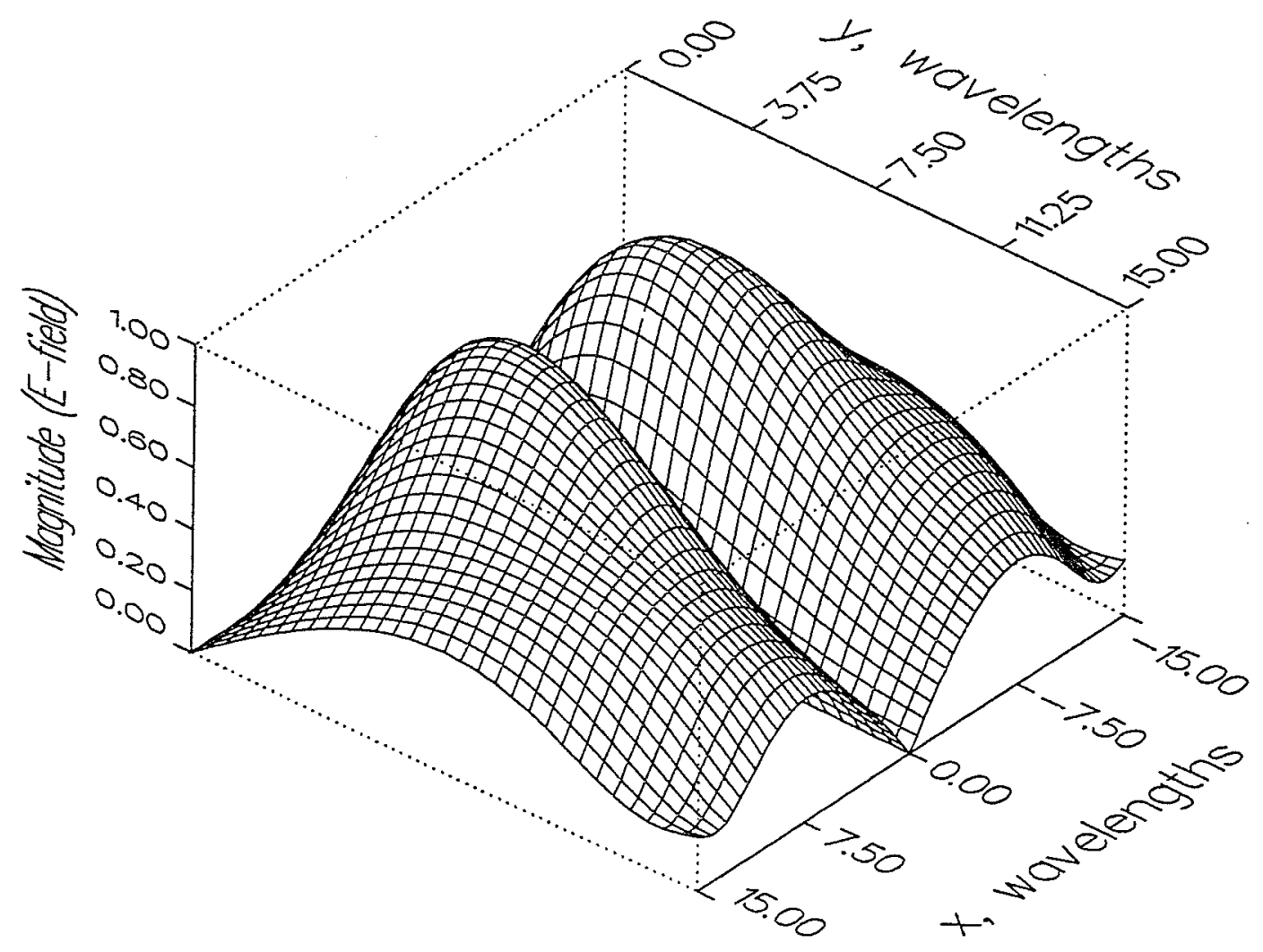

Fig. 3.11b : The field component $E_{y}$ over the aperture plane of a wide angle conical reflector $\left(\nu=75^{\circ}\right)$, at a distance $\mathrm{z}=$ const $=150.0 \lambda$ from the apex. 


\section{CHAPTER IV}

\section{The Performance Of Conical Reflectors With A Microstrip Comb Line Array Feed}

\subsection{Introduction}

The fields in the focal region of conical reflectors illuminated by a linearly polarized plane wave have been considered in $[46,58]$. It was shown that for large angle cones, both cross-polar and axial components are negligibly small near the axis. Thus, for antenna with a moderate cross-polar radiation, a linearly polarized array such as dipole array can be used as their line source $[57,58]$.

For practical implementation of dipole arrays a microstrip configuration may be used that facilitates the design and fabrication. For wide angle cones the linear array can be implemented using comb line [60,61] radiating in both directions, Fig. 4.1 a. Here, the radiation intensity is proportional to the dipole widths and the focal region field along with the traveling line antenna properties can be used to determine their location and width. For the $90^{\circ}$ cone, the dual polarized dipole array can be designed by a combination of comb line and the proximity coupled dipole arrays shown in Fig. $4.1 \mathrm{~b}$. The coupling coefficients can be controlled by the dipole separation from the feed line [59]. Alternative designs using undulated lines, with or without a conducting ground plane is also possible $[62,63,64]$ and can be designed using the traveling wave antenna theory. In this work we have studied the performance of conical reflectors with a line source feed which is made of an array of microstrip comb lines. In this work we have used the focal region field to determine the excitation intensity of each microstrip comb line stub, i.e. array elements, at their location. The selected array excitations along with the traveling line antenna properties can be used to determine the stub locations and their widths. 


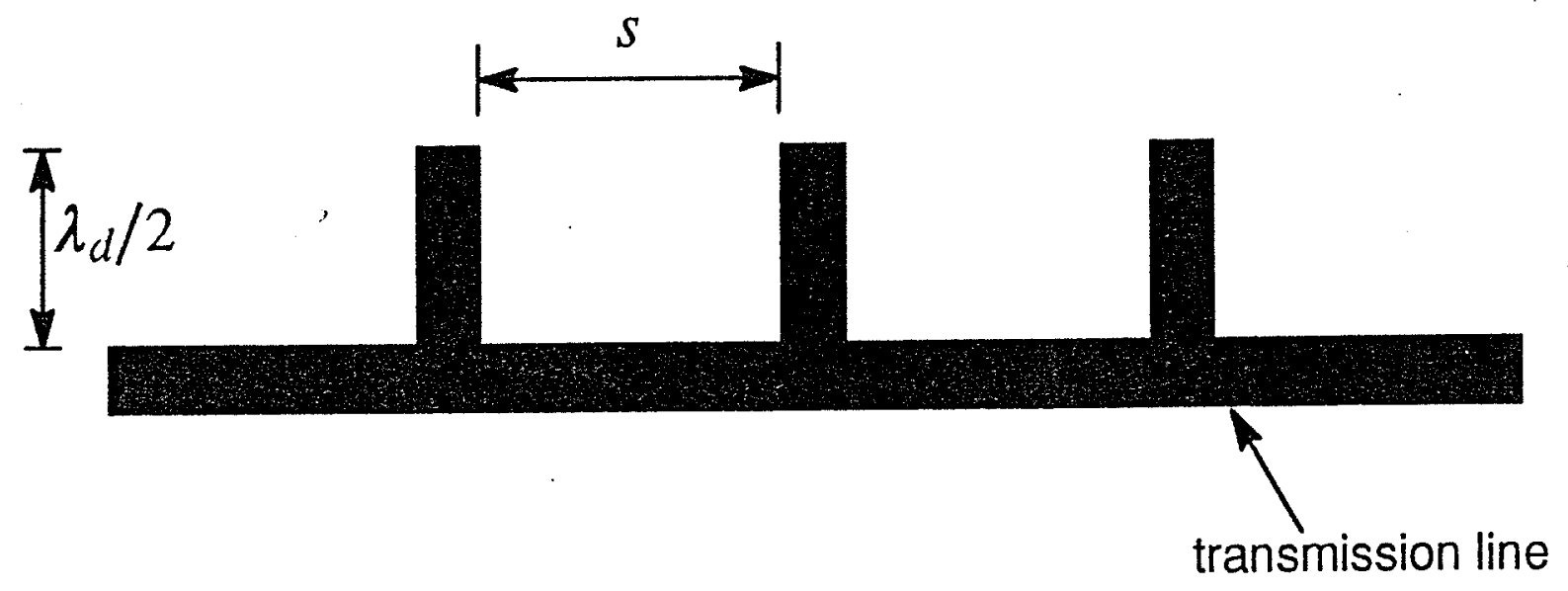

Fig. 4.1a: Geometry of comb line array.

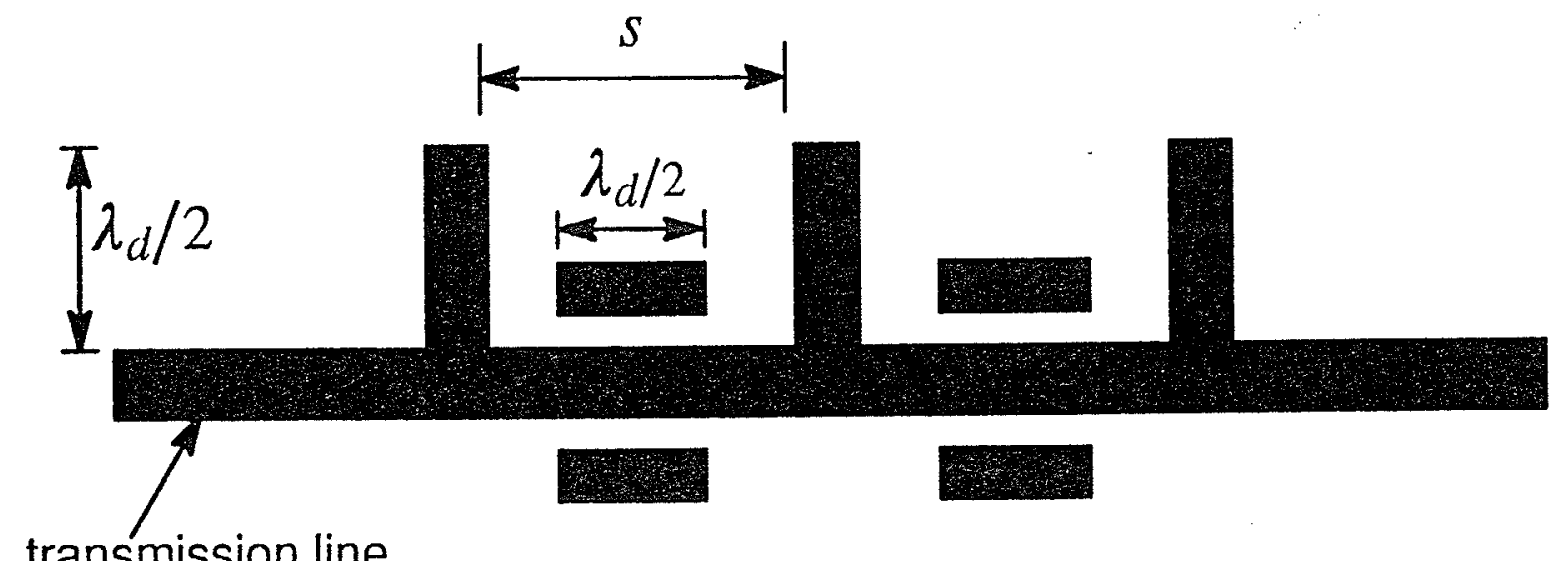

transmission line

Fig. 4.1b : Geometry of a dual polarized array using a comb line and proximity coupled diploe array. 
In the following section, The expression for the far field patterns of conical reflectors with a line source feed in the form of two microstrip comb line arrays, are derived and computed using a numerical integration of the physical optics currents. For selected cases numerical data for both co- and cross-polar patterns are generated to examine the conical reflector performance with these simple feed.

\subsection{Wide Angle Cone with Linearly Polarized Microstrip Comb Line Array Feed}

Consider a line source feed in the form of two microstrip comb line arrays (Right comb line and Left comb line), radiating in both directions, oriented parallel to the $x$ axis and located in both sides of the $y$ axis of the coordinate system of Fig. 4.2. The incident field from the feed induces current distributions on the reflector surface, which is assumed to be perfectly conducting.

The direction of the array beam $\theta_{o}$, which is equal to the contained cone angle $2 v$ can be write as $[65]$;

$$
\cos \theta_{o}=\frac{\lambda}{\lambda_{g f}}-\frac{\lambda}{s}
$$

where;

$\lambda$ is free space wavelength for a given operating frequency $f_{o}$, and,

$\lambda_{g f}$ is the guide wavelength of the feeder line, which are related for a given microstrip material $\varepsilon_{r}[66]$ as ;

$$
\lambda_{g f}=\frac{\lambda}{\sqrt{\varepsilon_{r}}}
$$

Using (4.2) in (4.1), we can obtain $s$ which is the separation between adjacent stubs.

Having calculated the excitation coefficients according to the focal region field [58], one can obtain (Appendix C ) the electrical quantities, and the physical dimensions of the microstrip comb line array. 


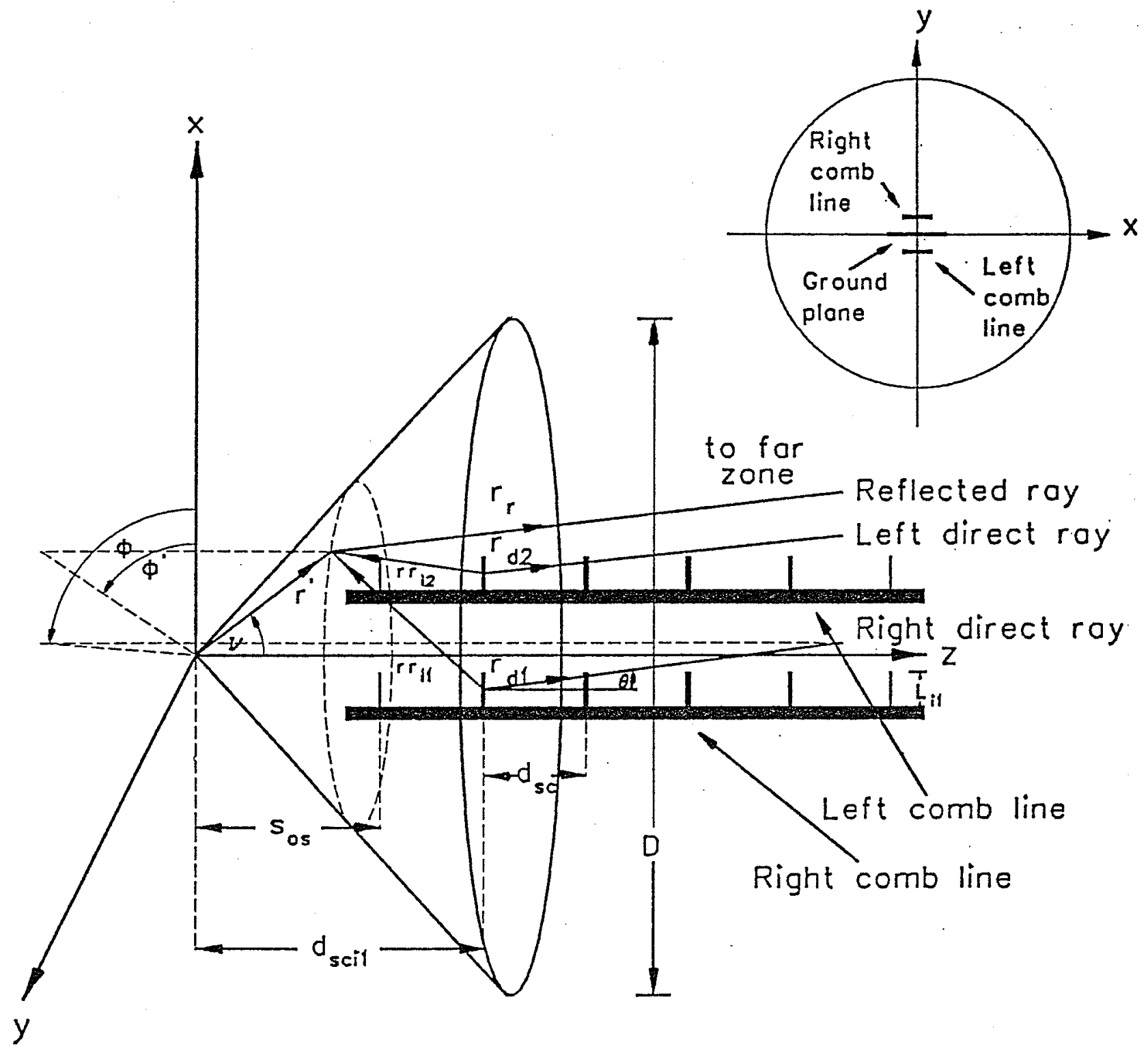

Fig. 4.2 : Conical reflector having a line source feed in the form of two microstrip comb line arrays, oriented parallel to the $x$-axis. 
The incident magnetic field from the feed (Appendix D ) on the conical reflector, can be written in the rectangular coordinates as ;

$$
\mathbf{H}^{i n c}=\frac{j k h}{\pi Z_{o}} \sum_{j=1}^{2} \sum_{i=1}^{N_{x}} A_{s c i j}\left[(q w x) \hat{a}_{x}+(q w y) \hat{a}_{y}+(q w z) \hat{a}_{z}\right]
$$

in which ;

$$
\begin{aligned}
& A_{s c i j}=E_{y i j} w_{i j} e^{-j k d_{s i j} \cos \theta_{o}} W_{s c i j} \\
& q w x=A J J\left[-\sin \theta_{i j}^{\prime} \cos \theta_{i j}^{\prime} \cos \phi_{i j}^{\prime}\right]+A R R\left[-\cos \theta_{i j}^{\prime}\right] \\
& q w y=A J J\left(-\sin \theta_{i j}^{\prime} \cos \theta_{i j}^{\prime} \sin \phi_{i j}^{\prime}\right)+A R R(0.0) \\
& q w z=A J J\left(+\sin \theta^{\prime 2}{ }_{i j}\right)+A R R\left[+\sin \theta_{i j}^{\prime} \cos \phi_{i j}^{\prime}\right] \\
& A J J=j\left[\sin A_{i j}+\left|\bar{\Gamma}_{i j}\right| \sin B_{i j}\right] \\
& A R R=k\left(\varepsilon_{e f f i j}\right)^{1 / 2}\left[\cos A_{i j}-\left|\bar{\Gamma}_{i j}\right| \cos B_{i j}\right] \\
& A_{i j}=k\left[+\sin \theta_{i j}^{\prime} \cos \phi_{i j}^{\prime}-\left(\varepsilon_{e f f i j}\right)^{1 / 2}\right] \frac{L_{i j}}{2} \\
& B_{i j}=\left[A_{i j}-2 k\left(\varepsilon_{e f f i j}\right)^{1 / 2} \frac{L_{i j}^{\prime}}{2}\right] \\
& W_{s c i j}=\frac{\sin Y^{\prime}}{Y^{\prime}} \frac{\sin Z^{\prime}}{Z^{\prime}} h w p \frac{e^{-j k r_{i j}^{\prime}}}{r_{i j}^{\prime}} \\
& Y^{\prime}=k h \sin \theta_{i j}^{\prime} \sin \phi_{i j}^{\prime} \\
& Z^{\prime}=\frac{1}{2} k w_{i j} \cos \theta_{i j}^{\prime} \\
& h w p=e^{+j k\left[h \sin \theta_{i j}^{\prime} \sin \phi_{i j}^{\prime}+\frac{1}{2} w_{i j} \cos \theta_{i j}^{\prime}\right]}
\end{aligned}
$$




$$
\begin{aligned}
& r_{i j}^{\prime}=\left[\left(\rho^{\prime} \cos \phi^{\prime}-x_{i j}\right)^{2}+\left(\rho^{\prime} \sin \phi^{\prime}-y_{i j}\right)^{2}+\left(\rho^{\prime} \cot v-d_{s c i j}\right)^{2}\right]^{1 / 2} \\
& \rho_{i j}^{\prime}=\left[\left(\rho^{\prime} \cos \phi^{\prime}-x_{i j}\right)^{2}+\left(\rho^{\prime} \sin \phi^{\prime}-y_{i j}\right)^{2}\right]^{1 / 2} \\
& \sin \theta_{i j}^{\prime}=\rho_{i j}^{\prime} / r_{i j}^{\prime} \\
& \cos \theta_{i j}^{\prime}=\left(\rho^{\prime} \cot v-d_{s c i j}\right) / r_{i j}^{\prime} \\
& \sin \phi_{i j}^{\prime}=\left(\rho^{\prime} \sin \phi^{\prime}-y_{i j}\right) / \rho_{i j}^{\prime} \\
& \cos \phi_{i j}^{\prime}=\left(\rho^{\prime} \cos \phi^{\prime}-x_{i j}\right) / \rho_{i j}^{\prime}
\end{aligned}
$$

with the term $e^{-j k d_{x i j} \cos \theta_{o}}$ being the array scanning factor.

Using (4.3) and (2.6) in (2.5), one obtains the components of the surface current density as ;

$$
\begin{aligned}
& J_{x i j}=\frac{j 2 k h \sin v}{\pi Z_{o}} \sum_{j=1}^{2} \sum_{i=1}^{N_{s c}} A_{s c i j}\left[-\operatorname{cotv} \sin \phi^{\prime} q w z-q w y\right] \\
& J_{y i j}=\frac{j 2 k h \sin v}{\pi Z_{o}} \sum_{j=1}^{2} \sum_{i=1}^{N_{s c}} A_{s c i j}\left[+\operatorname{cotv} \cos \phi^{\prime} q w z+q w x\right] \\
& J_{z i j}=\frac{j 2 k h \sin v}{\pi Z_{o}} \sum_{j=1}^{2} \sum_{i=1}^{N_{s c}} A_{s c i j}\left[-\operatorname{cotv} \cos \phi^{\prime} q w y+\operatorname{cotv} \sin \phi^{\prime} q w x\right]
\end{aligned}
$$

Substituting (4.11) in (3.1), we can write the contribution of the reflected rays from the right half and the left half of the reflector in the far-zone as ;

$$
\begin{aligned}
\mathbf{E}_{R e f r}=\frac{-j k Z_{o}}{4 \pi R} e^{-j k R} \int_{0}^{D / 2 \pi} \int_{0}\{ & {\left[\cos \theta \cos \phi J_{x i 1}+\cos \theta \sin \phi J_{y i 1}-\sin \theta J_{z i 1}\right] \hat{a}_{\theta} } \\
+ & {\left.\left[-\sin \phi J_{x i 1}+\cos \phi J_{y i 1}\right] \hat{a}_{\phi}\right\} } \\
& e^{j k \rho^{\prime}\left[\sin \theta \cos \left(\phi-\phi^{\prime}\right)+\cot v \cos \theta\right]} \rho^{\prime} \operatorname{cosecv} d \rho^{\prime} d \phi^{\prime}
\end{aligned}
$$




$$
\begin{aligned}
\mathbf{E}_{R e f l}=\frac{-j k Z_{o}}{4 \pi R} e^{-j k R} \int_{0}^{D / 2} \int_{\pi}^{2 \pi}\{ & {\left[\cos \theta \cos \phi J_{x i 2}+\cos \theta \sin \phi J_{y i 2}-\sin \theta J_{z i 2}\right] \hat{a}_{\theta} } \\
& \left.+\left[-\sin \phi J_{x i 2}+\cos \phi J_{y i 2}\right] \hat{a}_{\phi}\right\} \\
& e^{j k \rho^{\prime}\left[\sin \theta \cos \left(\phi-\phi^{\prime}\right)+\cot v \cos \theta\right] \rho^{\prime} \operatorname{cosec} v d \rho^{\prime} d \phi^{\prime}}
\end{aligned}
$$

Following the same procedure as in [10], we can write the contribution of the direct rays (Fig. 4.2) from the feed (right and left comb line arrays) in the far-zone as ;

$$
\begin{aligned}
\mathbf{E}_{\text {Dir }}=\frac{-j k Z_{o}}{4 \pi R} e^{-j k R} \frac{-4 h}{Z_{o}} & \sum_{j=1}^{2} \sum_{i=1}^{N_{s c}} E_{y i j} w_{i j} e^{-j k d_{s i j} \cos \theta_{o}} . \\
Q Q_{i j} & \left\{[B J J(0.0)+B R R(\cos \theta \sin \phi)] \hat{a}_{\theta}\right. \\
+ & {\left.[B J J(\sin \theta)+B R R(\cos \phi)] \hat{a}_{\phi}\right\} }
\end{aligned}
$$

where;

$$
\begin{aligned}
& Q Q_{i j}=\frac{\sin Y Y}{Y Y} \frac{\sin Z Z}{Z Z} v w p s w p \\
& Y Y= k h \sin \theta \sin \phi \\
& Z Z= \frac{1}{2} k w_{i j} \cos \theta \\
& v w p= e^{+j k\left[h \sin \theta \sin \phi+\frac{1}{2} w_{i j} \cos \theta\right]} \\
& s w p=e^{+j k}\left[x_{i j} \sin \theta \cos \phi+y_{i j} \sin \theta \sin \phi+d_{s c i j} \cos \theta\right] \\
& B J J=j\left[\sin C_{i j}+\left|\bar{\Gamma}_{i j}\right| \sin D_{i j}\right]
\end{aligned}
$$




$$
\begin{aligned}
& B R R=k\left(\varepsilon_{e f f i j}\right)^{1 / 2}\left[\cos C_{i j}-\left|\bar{\Gamma}_{i j}\right| \cos D_{i j}\right] \\
& C_{i j}=k\left[\sin \theta \cos \phi-\left(\varepsilon_{e f f i j}\right)^{1 / 2}\right] \frac{L_{i j}}{2} \\
& D_{i j}=\left[C_{i j}-2 k\left(\varepsilon_{e f f i j}\right)^{1 / 2} \frac{L_{i j}^{\prime}}{2}\right]
\end{aligned}
$$

in which ;

$$
d_{s c i j}=s_{o s}+(i-1) d_{s c}
$$

$v$ is the cone half angle,

$\theta_{o}$ is the array scanning angle,

$N_{s c}$ is the number of stubs in each comb line array,

$L_{i j}, L_{i j}^{\prime}$ length of microstrip conductor and 'end effect', respectively of the ijth stub,

$h$ is the substrate thickness,

$\varepsilon_{e f f i j}$ is the effective dielectric constant of the ijth stub,

$\left|\bar{\Gamma}_{i j}\right|, w_{i j}$ are the reflection coefficient and the effective width respectively, of the ijth stub, and,

$E_{y i j}$ being the actual or the truncated focal region field distribution.

The radiation characteristics of the conical reflector can be determined from these expressions.

\subsection{Numerical Results and Discussions for Far Field Patterns}

In the examples to follow, a wide angle cone is investigated. In each case an appropriate comb line array is selected and the reflector far field data is computed. The number of elements in each comb line array is selected such that, the array length equals approximately the geometrical optics line source length [70]. The comb line 
array used as a line source feed, designed to operate at center frequency $f_{o}=6 \mathrm{GHZ}$. The substrate assumed to be of the type with relative permittivity $\varepsilon_{r}=2.52$ and thickness $h=1.585 \mathrm{~mm}$. In these examples, we assume that the feed to the comb line array is connected to the end far from the apex, while the matched load is connected to the end near to the apex.

Figs. 4.3, 4.4 and 4.5 show the behavior of the actual and hypothetical normalized field distribution along the aperture of comb line array feed, for various $P_{l}$ ( percentage of the incident power dissipated on the matched feed located at the end of the comb line ). Figs 4.6, 4.7 and 4.8 show the stub width and conductance corresponding to the field distribution shown in Figs. 4.3, 4.4 and 4.5 , respectively. From these figures. we can conclude that, the stub conductance and width are decreased with the increase of $P_{l}$.

For the designed operating frequency $f_{o}=6 \mathrm{GHZ}$, and with the assumed substrate relative permittivity $\varepsilon_{r}=2.52$ and thickness $h=1.585 \mathrm{~mm}$, the separation between adjacent stubs $(s)$ is $21.35 \mathrm{~mm}$. It is clear from Fig. 4.6 that, the maximum stub widths for the A.F.R.F and H.F.R.F. are $16.82 \mathrm{~mm}$ and $17.85 \mathrm{~mm}$, respectively, which provide an inter stub separation of about $3.50 \mathrm{~mm}$. Larger separation can be obtained by decreasing the stub widths, this provides an adjacent separation between adjacent stubs to minimize their mutual coupling. In Figs. 4.7 and 4.8, this is achieved by increasing the percentage power loss in the matched load, where the stub widths are $15.0 \mathrm{~mm}$ and $10.0 \mathrm{~mm}$, respectively. In the latter case, in Fig. 4.8, the stub widths of $10.0 \mathrm{~mm}$ increases the inter stub separation to about $12.00 \mathrm{~mm}(\lambda / 4)$, which is an adequate separation to eliminate mutual coupling.

Figs. 4.9 to 4.14 show the behavior of the co-polar and cross-polar field components for a wide angle reflector with an aperture diameter of $30.0 \lambda$, having different array element excitations, for various $P_{l}$. The $E_{\theta}$ component is computed in the $E$ plane, $\left(\phi=90^{\circ}\right)$, while the $E_{\phi}$ component is computed in the $H$-plane, $\left(\phi=0^{\circ}\right)$. The cross-polar component is computed using Ludwig's [56] third definition in the plane, $\phi=45^{\circ}$, where it has maximum distribution. 
Figs. 4.9, 4.11 and 4.13 show the results for an array of microstrip comb line having the field excitation given according to the exact focal region field $\left(E=E_{\text {A.F.R.F }}\right)$ shown in Figs. 4.3, 4.4 and 4.5, respectively. Figs. 4.10, 4.12 and 4.14 show the results for the same array, but with the field excitation given according to the hypothetical focal region field $\left(E=E_{H . F R . F}\right)$ shown in Figs. 4.3, 4.4 and 4.5 , respectively.

From Figs. 4.13 and 4.14 it is clear that the peak cross-polarization is $-18 \mathrm{~dB}$ and the first side lobe level in the $H$-plane is $-19 \mathrm{~dB}$, while in the $E$-plane it is close to $-18 \mathrm{~dB}$. The results show that, the reflector performance is not compromized by selecting the simpler hypothetical focal region field of Fig. 4.5, instead of the original complex focal region field in Fig. 4.5. Also, the reflector performance has not deteriorated by increasing $P_{l}$ to decrease the stub widths of the comb line array.

In these examples, the use of microstrip comb line arrays provides far field patterns with satisfactory gain factors of about $70 \%$ and slight high cross-polarizations. The computed gain factors include the feed spillover power and the load loss but exclude the substrate losses.

The above results for wide angle cones indicate that microstrip comb line arrays can be used as feeds for conical reflectors. 


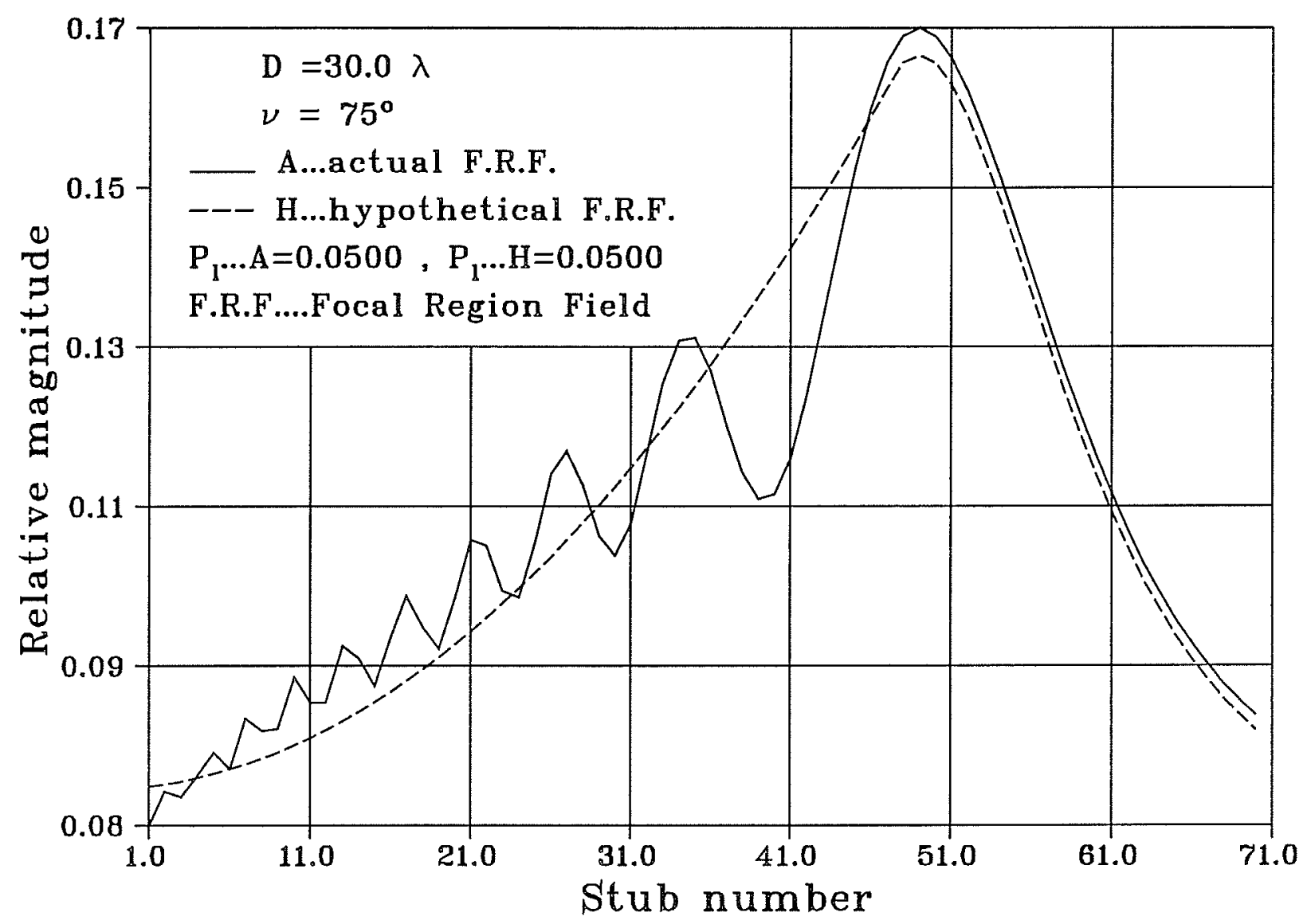

Fig. 4.3: The field distribution along the aperture of the microstrip comb line array elements, with max stub width $>15.0 \mathrm{~mm}, \mathrm{P}_{1}$ is the percentage load power. 


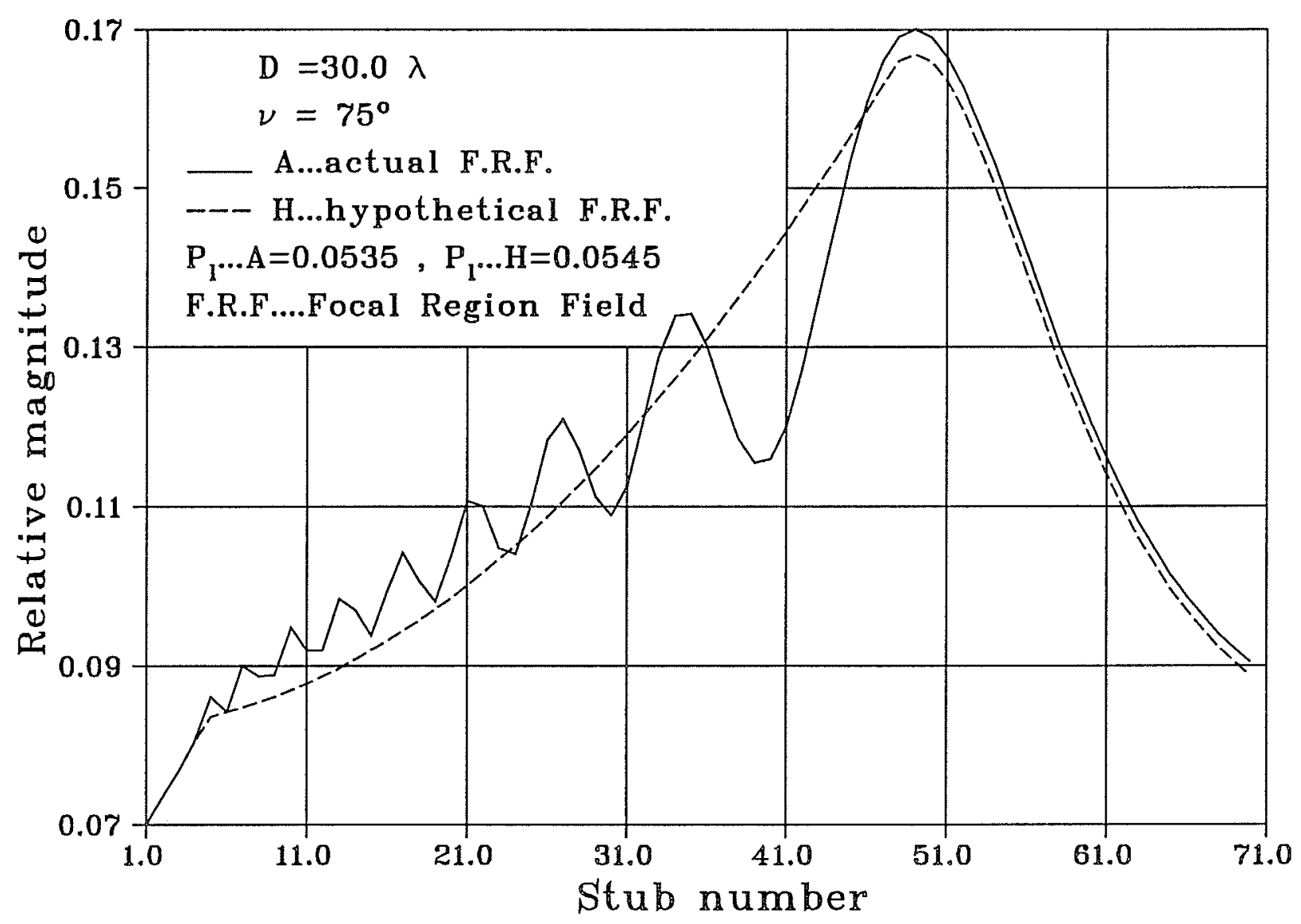

Fig. 4.4 : The field distribution along the aperture of the microstrip comb line array elements, with $\max$ stub width $=15.0 \mathrm{~mm}, P_{1}$ is the percentage load power. 


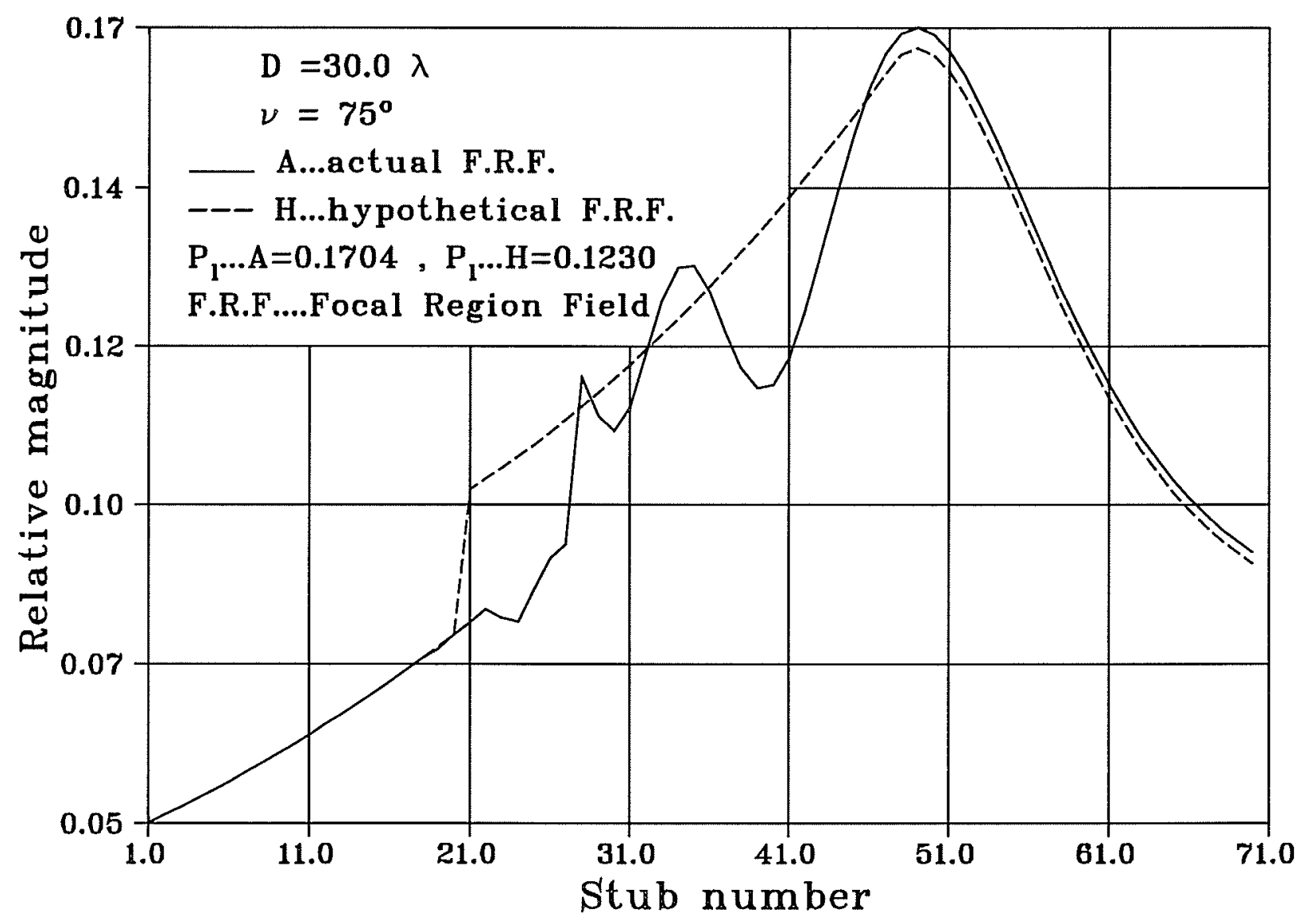

Fig. 4.5 : The field distribution along the aperture of the microstrip comb line array elements, with max stub width $=10.0 \mathrm{~mm}, \mathrm{P}_{1}$ is the percentage load power. 


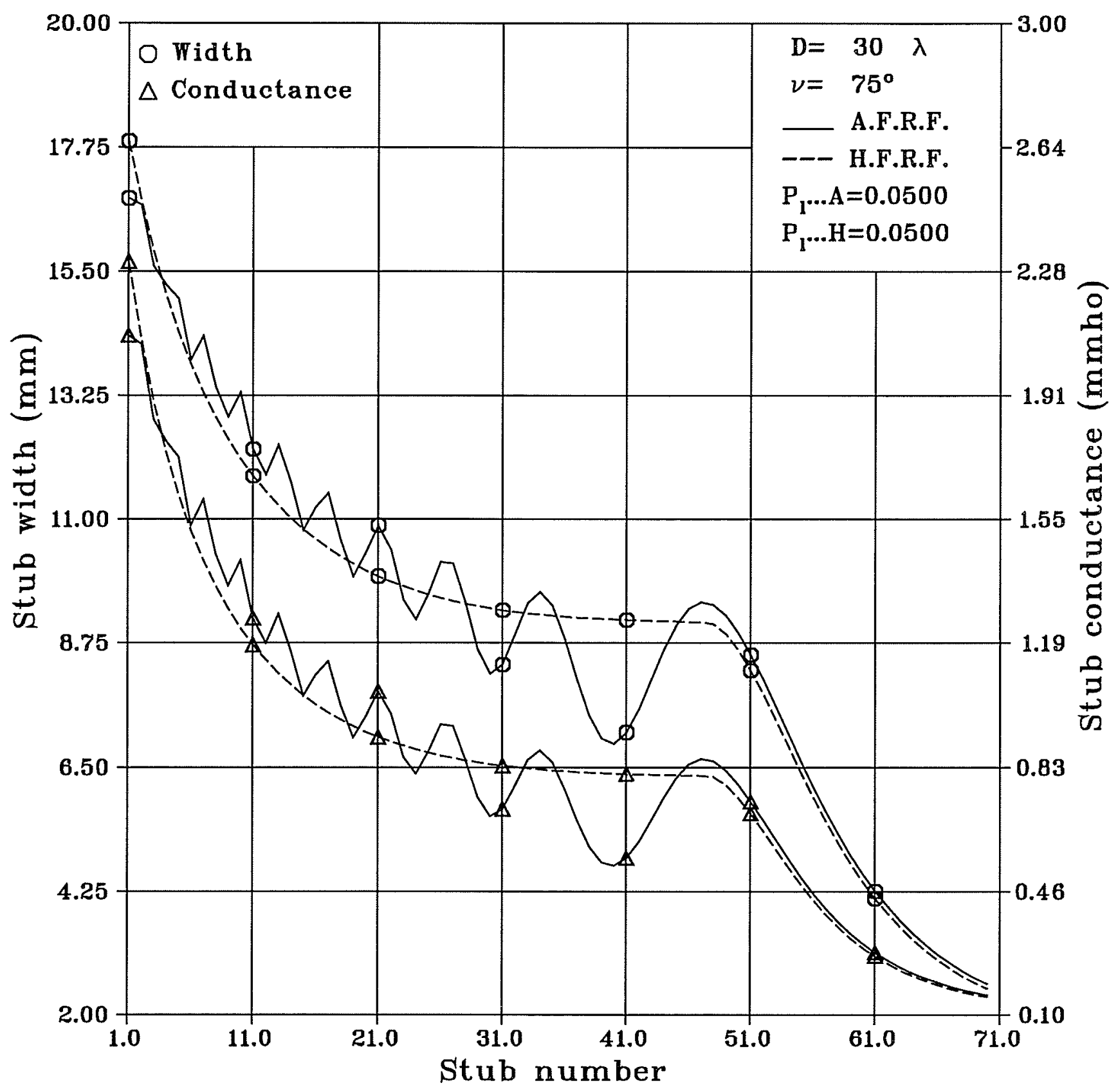

Fig. 4.6 : Stub width and conductance for the field distributions of Fig. 4.3, $P_{1}$ is the percentage load power. 


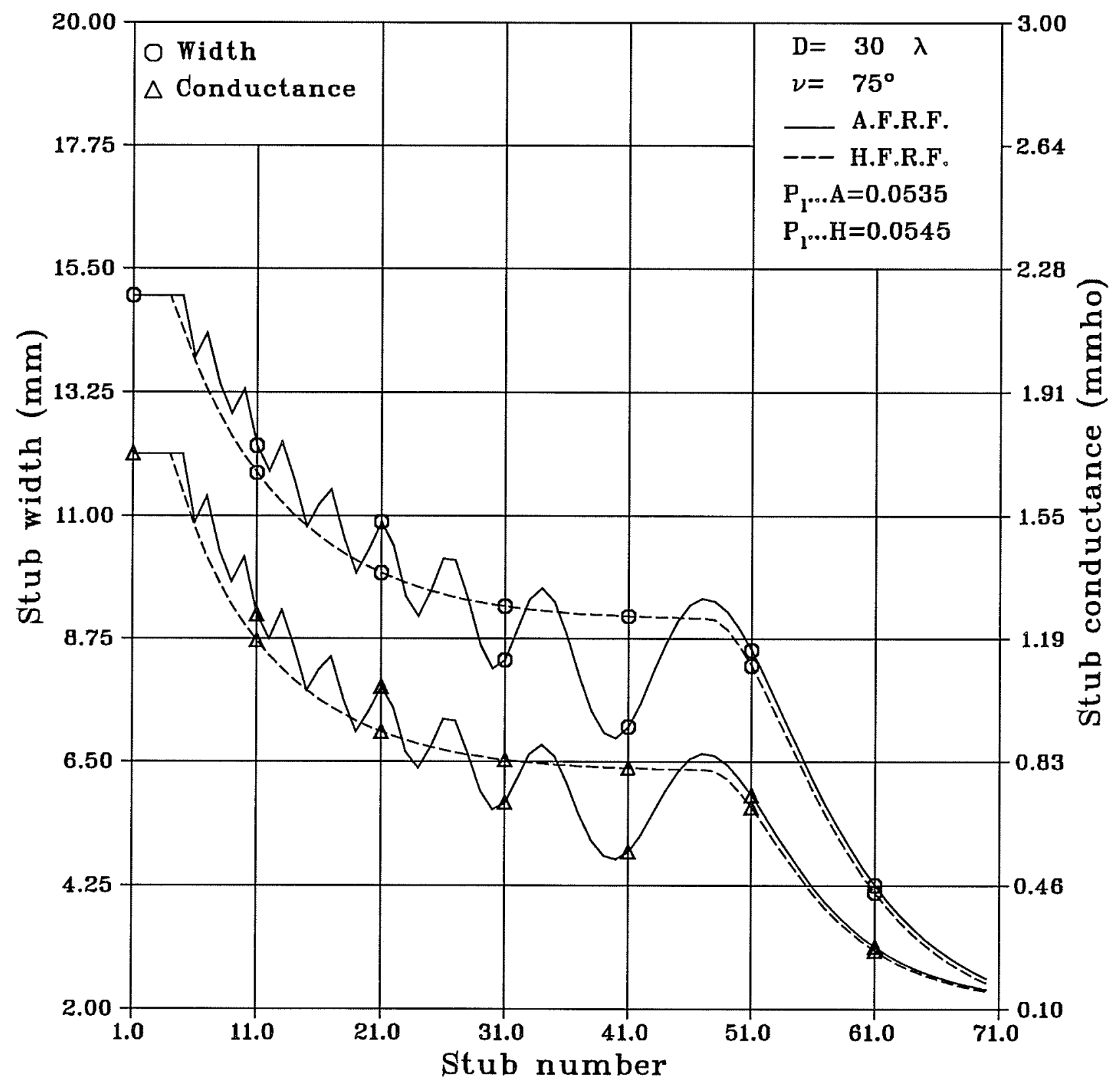

Fig. 4.7 : Stub width and conductance for the field distributions of Fig. 4.4, $\mathrm{P}_{1}$ is the percentage load power. 


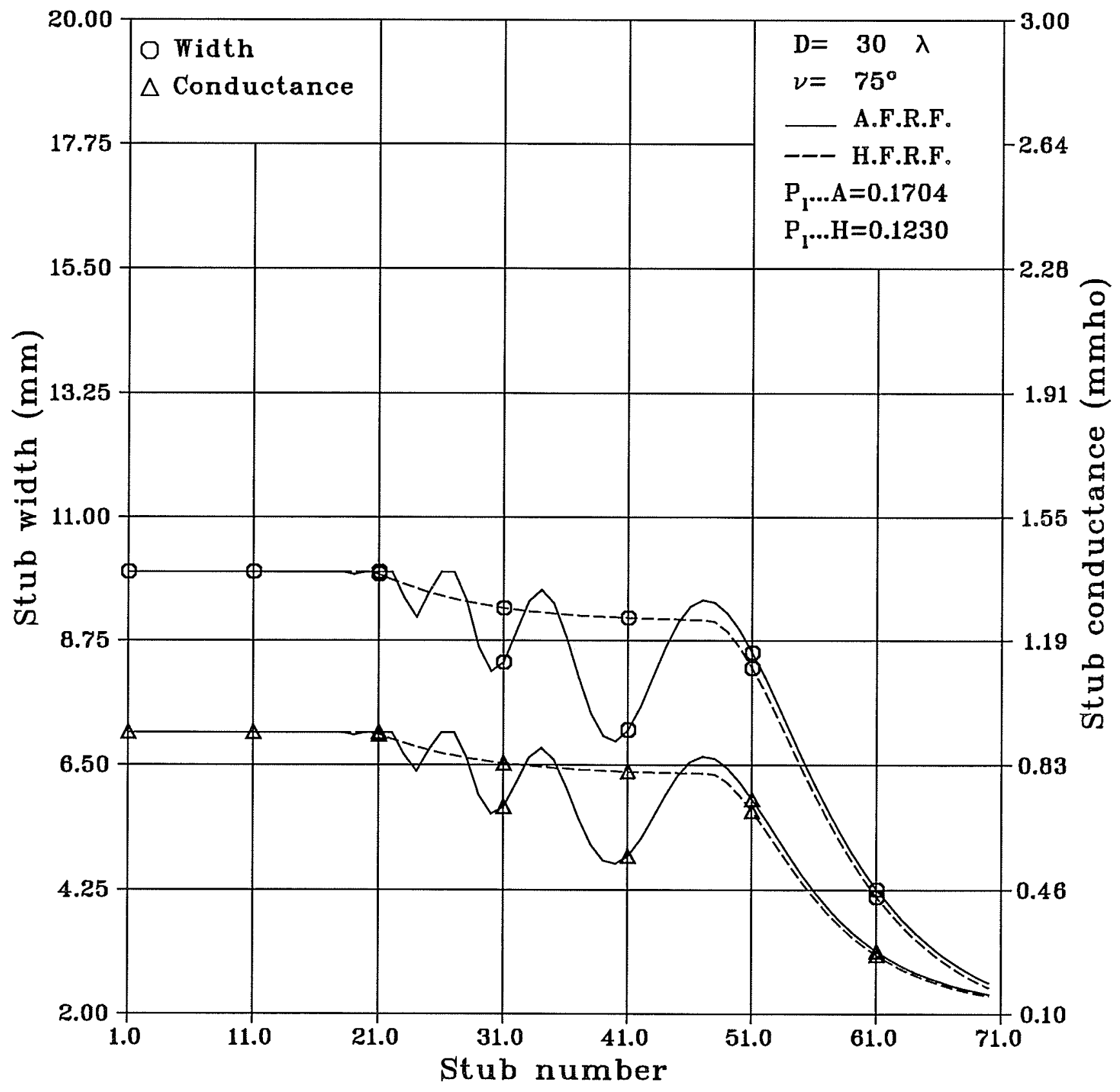

Fig. 4.8 : Stub width and conductance for the field distributions of Fig. 4.5, $\mathrm{P}_{1}$ is the percentage load power. 


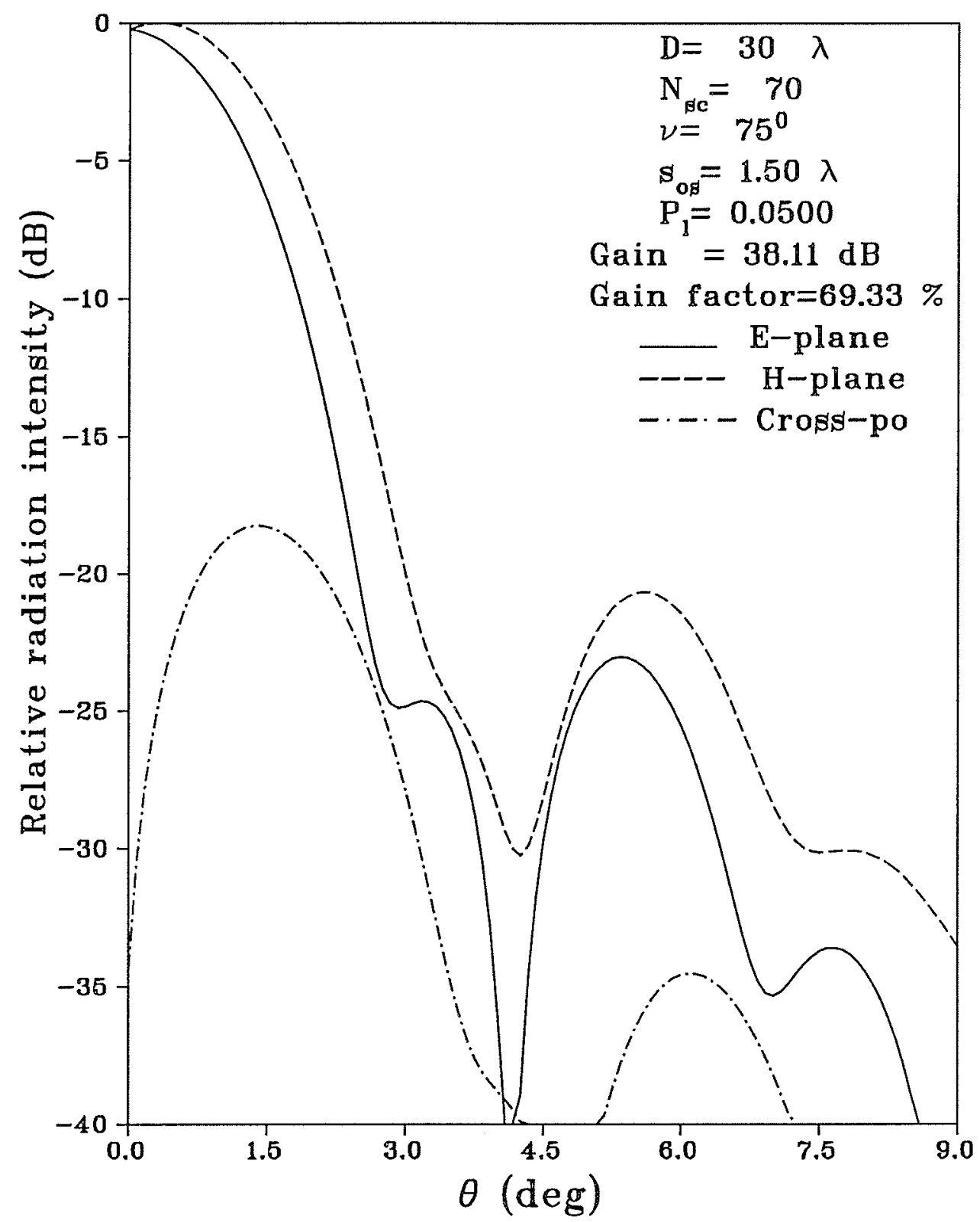

Fig. 4.9 : The radiation patterns of a wide angle conical reflector having a line source feed of two microstrip comb line arrays oriented parallel to the $\mathrm{x}$-axis, excitation according to actual focal field distribution of Fig. 4.3, $P_{1}$ is the percentage load power. 


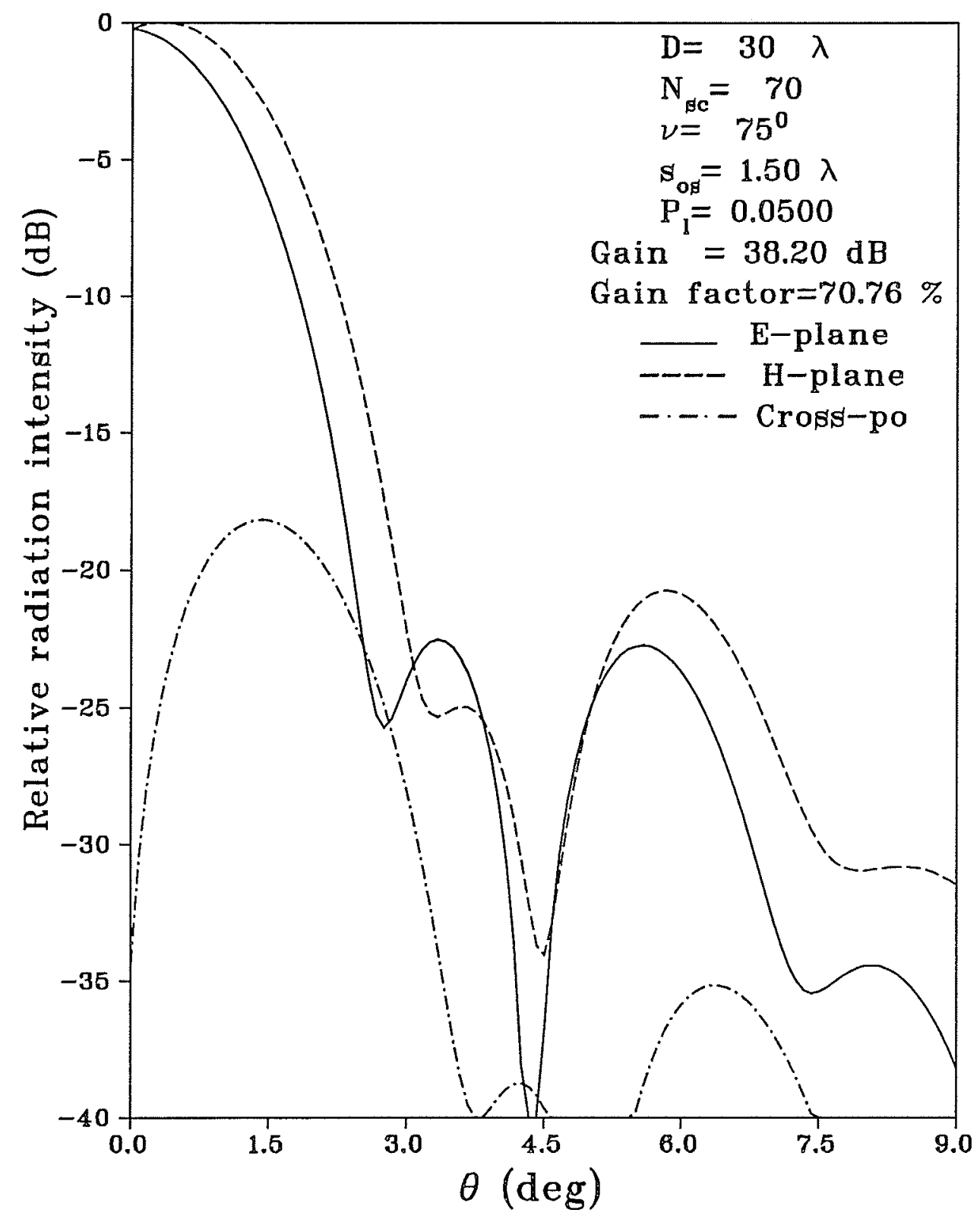

Fig. 4.10 : The radiation patterns of a wide angle conical reflector having a line source feed of two microstrip comb line arrays oriented parallel to the $\mathrm{x}$-axis, excitation according to a hypothetical focal field distrFig. 4.3, $\mathrm{P}_{1}$ is the percentage load power. 


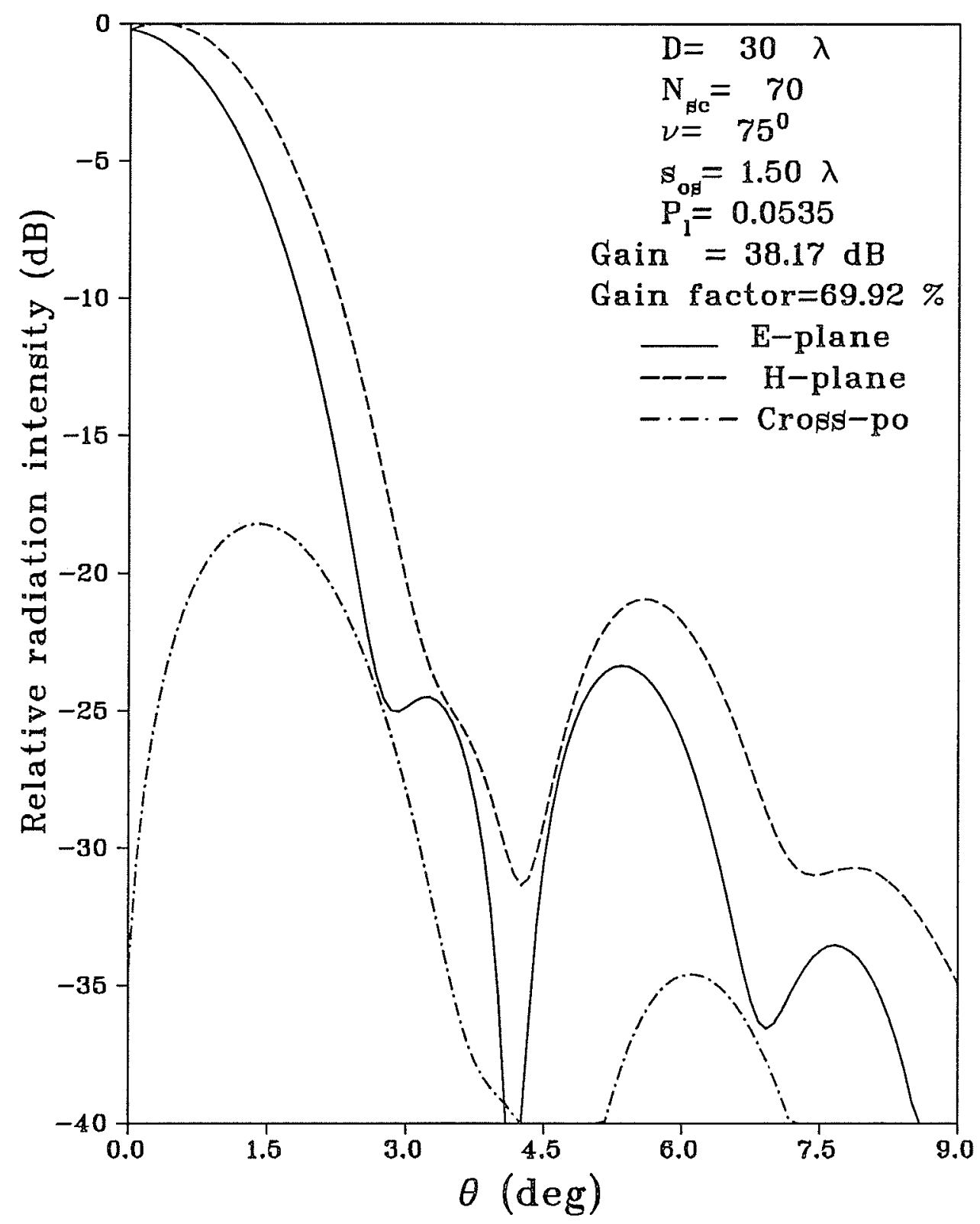

Fig. 4.11: The radiation patterns of a wide angle conical reflector having a line source feed of two microstrip comb line arrays oriented parallel to the $\mathrm{x}$-axis, excitation according to actual focal field distribution of Fig. 4.4, $\mathrm{P}_{1}$ is the percentage load power. 


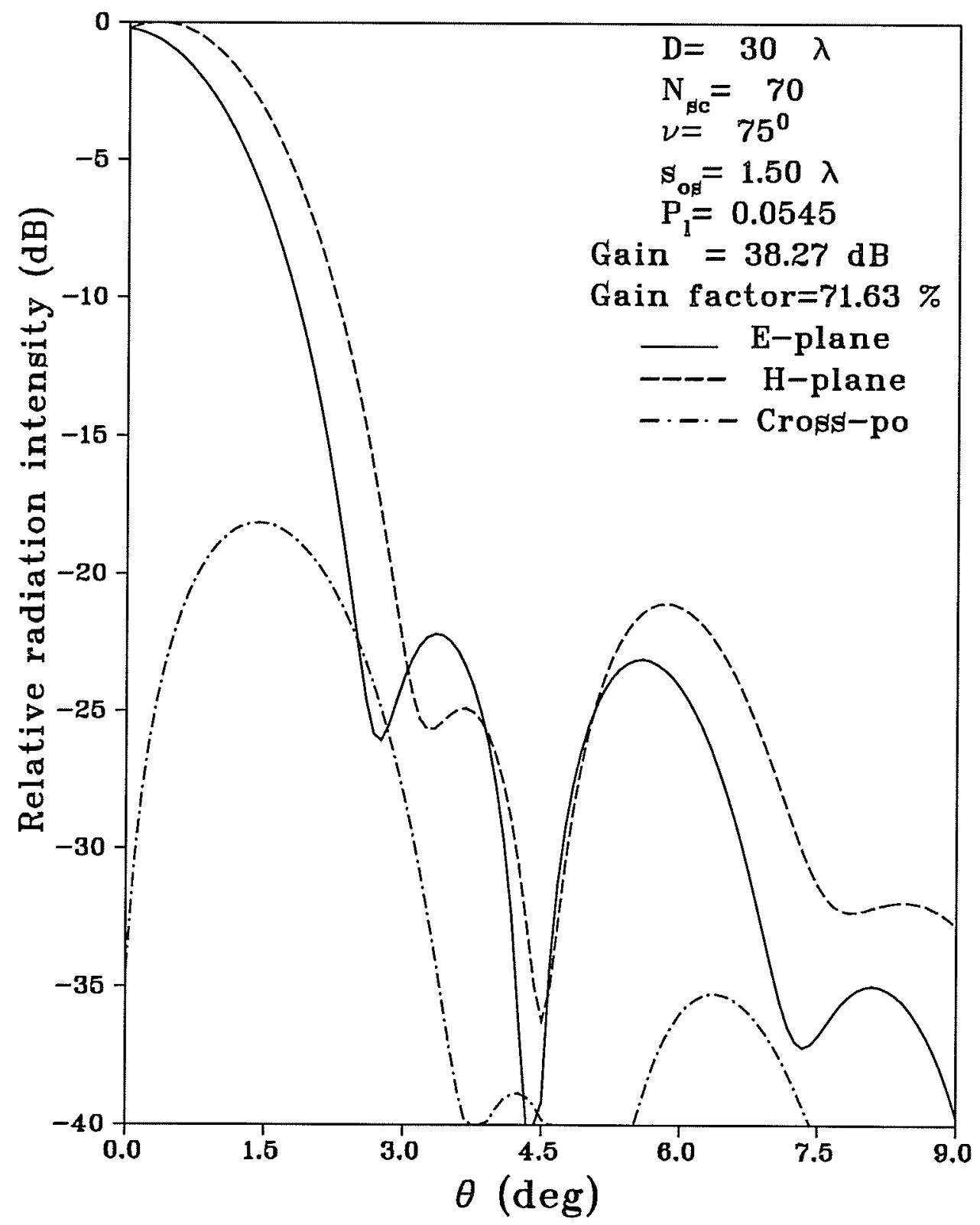

Fig. 4.12 : The radiation patterns of a wide angle conical reflector having a line source feed of two microstrip comb line arrays oriented parallel to the $x$-axis, excitation according to a hypothetical focal field distrFig. 4.4, $\mathrm{P}_{1}$ is the percentage load power. 


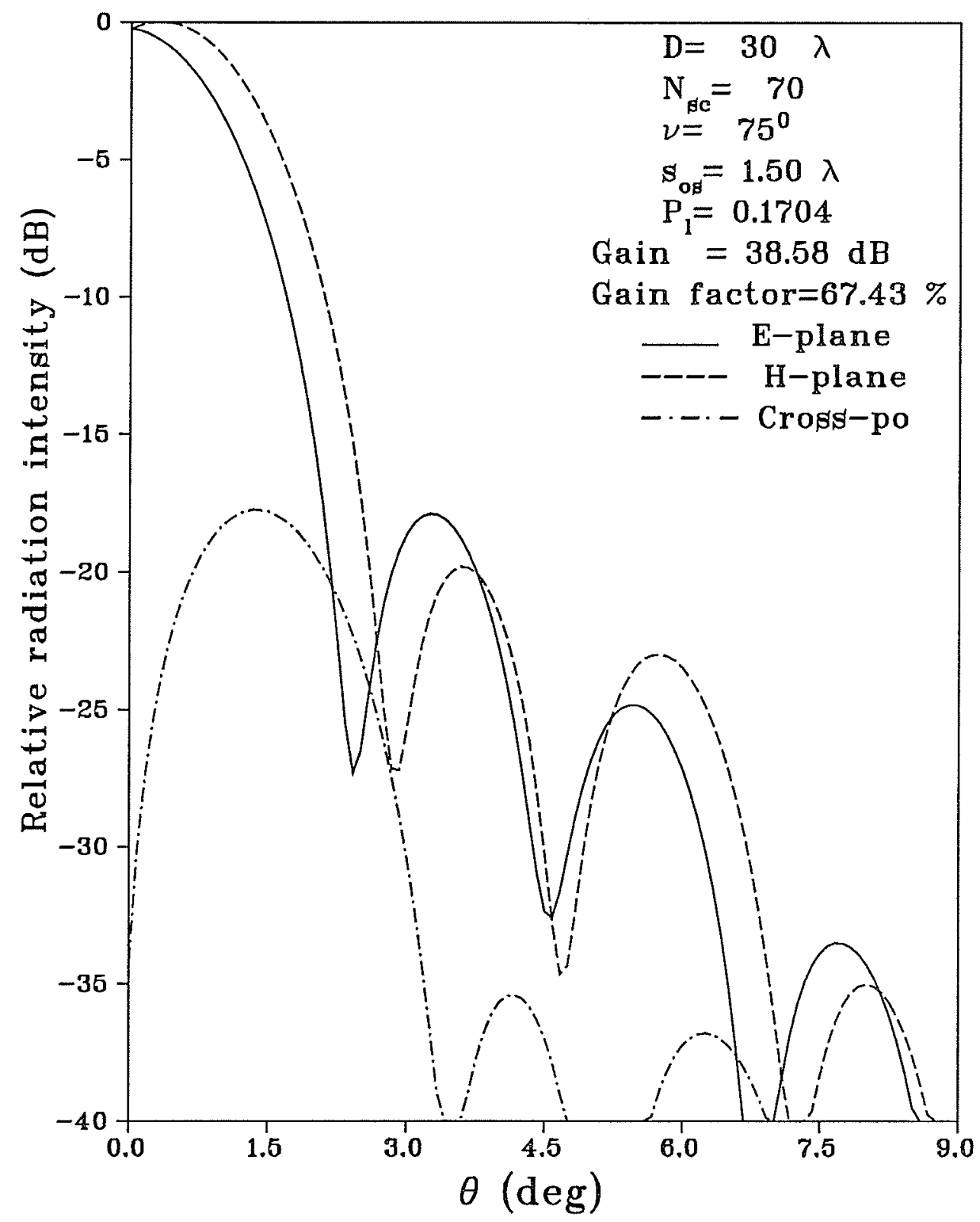

Fig. 4.13 : The radiation patterns of a wide angle conical reflector having a line source feed of two microstrip comb line arrays oriented parallel to the $\mathrm{x}$-axis, excitation according to actual focal field distribution of Fig. 4.5, $\mathrm{P}_{1}$ is the percentage load power. 


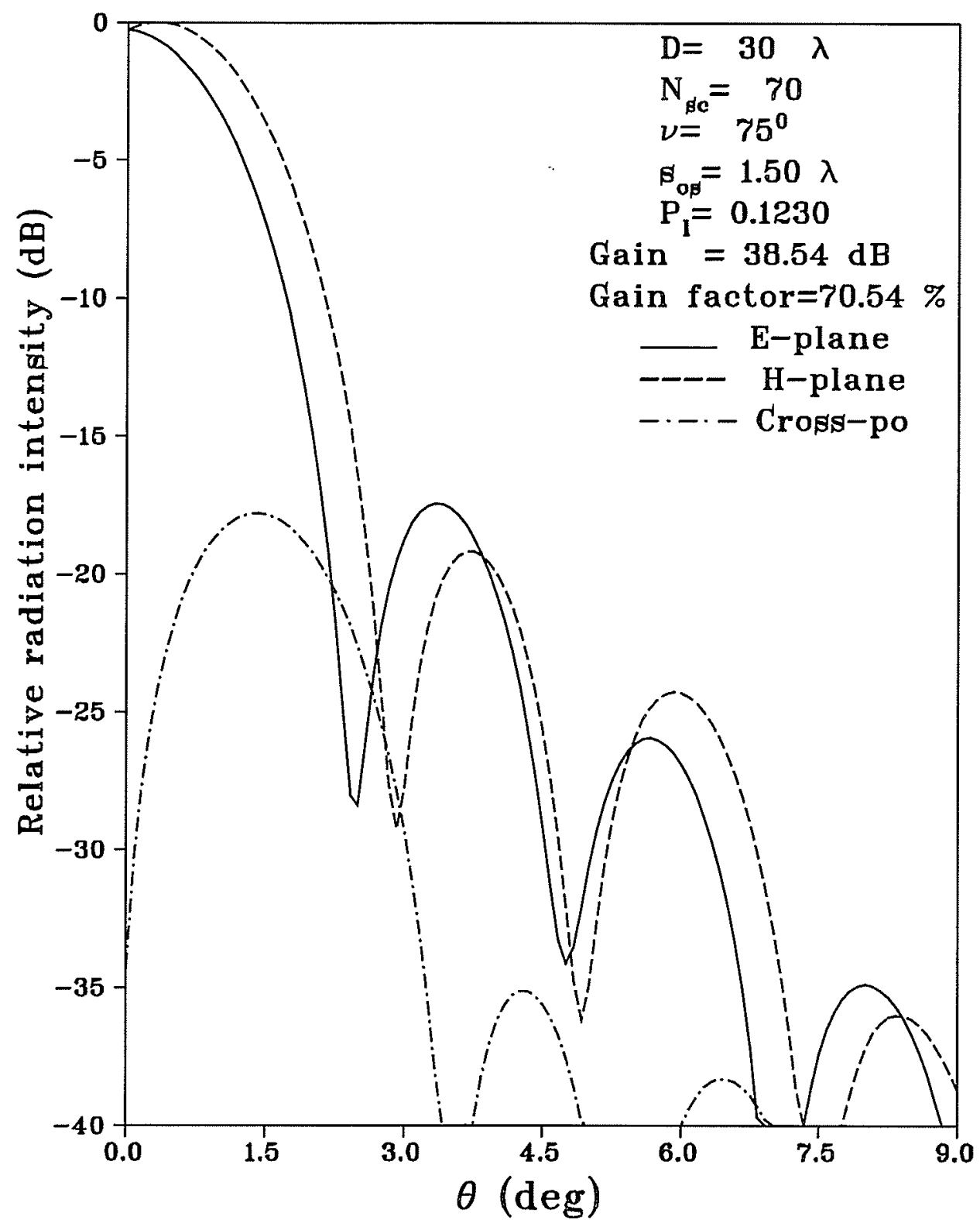

Fig. 4.14 : The radiation patterns of a wide angle conical reflector having a line source feed of two microstrip comb line arrays oriented parallel to the $x$-axis, excitation according to a hypothetical focal field distrFig. 4.5, $\mathrm{P}_{1}$ is the percentage load power. 


\section{CHAPTER V}

\section{Discussion and Recommendations for Future Work}

\subsection{Discussion}

The polarization nature of the field in the focal region of conical reflectors was characterized. It was shown that, near the focal line both cross-polar and axial field components are small and the required line source must be linearly polarized. However, over a cylindrical surface of a finite radius, the field distribution contained a significant axial field component $E_{z}$, especially when the total contained cone angle is $90^{\circ}$. These results indicated that for a line source of finite diameter, its surface field distribution must contain other field components to generate a linearly polarized farfield. Specifically, for wide angle cones, a linearly polarized array, such as a dipole array can be used as their line source. For narrow angle cones (around $90^{\circ}$ ), on the other hand, a dual polarized dipole must be used to provide both transverse and axial components of the field. When dipole excitations correspond to the focal region field, the reflector far field becomes linearly polarized with small cross-polarization field, to provide a linearly polarized far field.

For the wide angle cone case, a microstrip comb line array feed was demonstrated. A compact design was presented for a microstrip comb line array feed. An optimization technique was used to obtain the physical dimensions on one side of the substrate for the traveling wave array. Correction formulas were used to compensate for the effect of discontinuities in the structure and further correction was introduced to give a proper impedance match with the rest of the system by an admittance transformation. 


\subsection{Recommendation for Future Research Work}

A few valuable and interesting problems may be generated from this dissertation.

In the case of narrow angle cones, using the combination of comb line and proximity coupled dipole arrays as a line source feed for the $90^{\circ}$ cone, must be studied.

A practical implementation of dipole arrays as a microstrip configuration to facilitate the design and fabrication, should be made to evaluate the reflector performance.

Investigation of antenna performance over a practical bandwidth, must be considered. 


\section{Appendix A.}

\section{Near Fields of a Finite Dipole oriented along the x-axis and fed by a Sinusoidal Current Distribution}

Referring to Fig. A.1a and assuming a sinusoidal current distribution along the element given as ;

$$
\mathbf{I}_{e}\left(x^{\prime}, y^{\prime}, z^{\prime}\right)= \begin{cases}\hat{a}_{x} I_{o} \sin \left[k\left(\frac{l_{x}}{2}-x^{\prime}\right)\right] & 0 \leq x^{\prime} \leq+\frac{l_{x}}{2} \\ \hat{a}_{x} I_{o} \sin \left[k\left(\frac{l_{x}}{2}+x^{\prime}\right]\right] & -\frac{l_{x}}{2} \leq x^{\prime} \leq 0\end{cases}
$$

The magnetic vector potential A can be written as ;

$$
\mathbf{A}(x, y, z)=\frac{\mu}{4 \pi} \int_{C} \mathbf{I}_{e}\left(x^{\prime}, y^{\prime}, z^{\prime}\right) \frac{e^{-j k r}}{r} d l^{\prime}
$$

where $(x, y, z)$ represent the observation point coordinates, $\left(x^{\prime}, y^{\prime}, z^{\prime}\right)$ represent the coordinates of the source point, $r$ is the distance from any point in the source to the observation point, and path $\mathrm{C}$ is along the length of the source.

Substituting (A.1) into (A.2), the magnetic vector potential A can be written as ; $\mathbf{A}=\hat{a}_{x} A_{x}$

$$
\left.\begin{array}{rl}
=\hat{a}_{x} \frac{\mu I_{o}}{4 \pi}\left\{\int_{-\frac{l_{x}}{2}}^{0} \sin \left[k\left[\frac{l_{x}}{2}+x^{\prime}\right]\right] \frac{e^{-j k r}}{r} d x^{\prime}\right. \\
+\int_{0}^{+\frac{l_{x}}{2}} \sin \left[k\left(\frac{l_{x}}{2}-x^{\prime}\right)\right] \frac{e^{-j k r}}{r} d x^{\prime}
\end{array}\right\}
$$




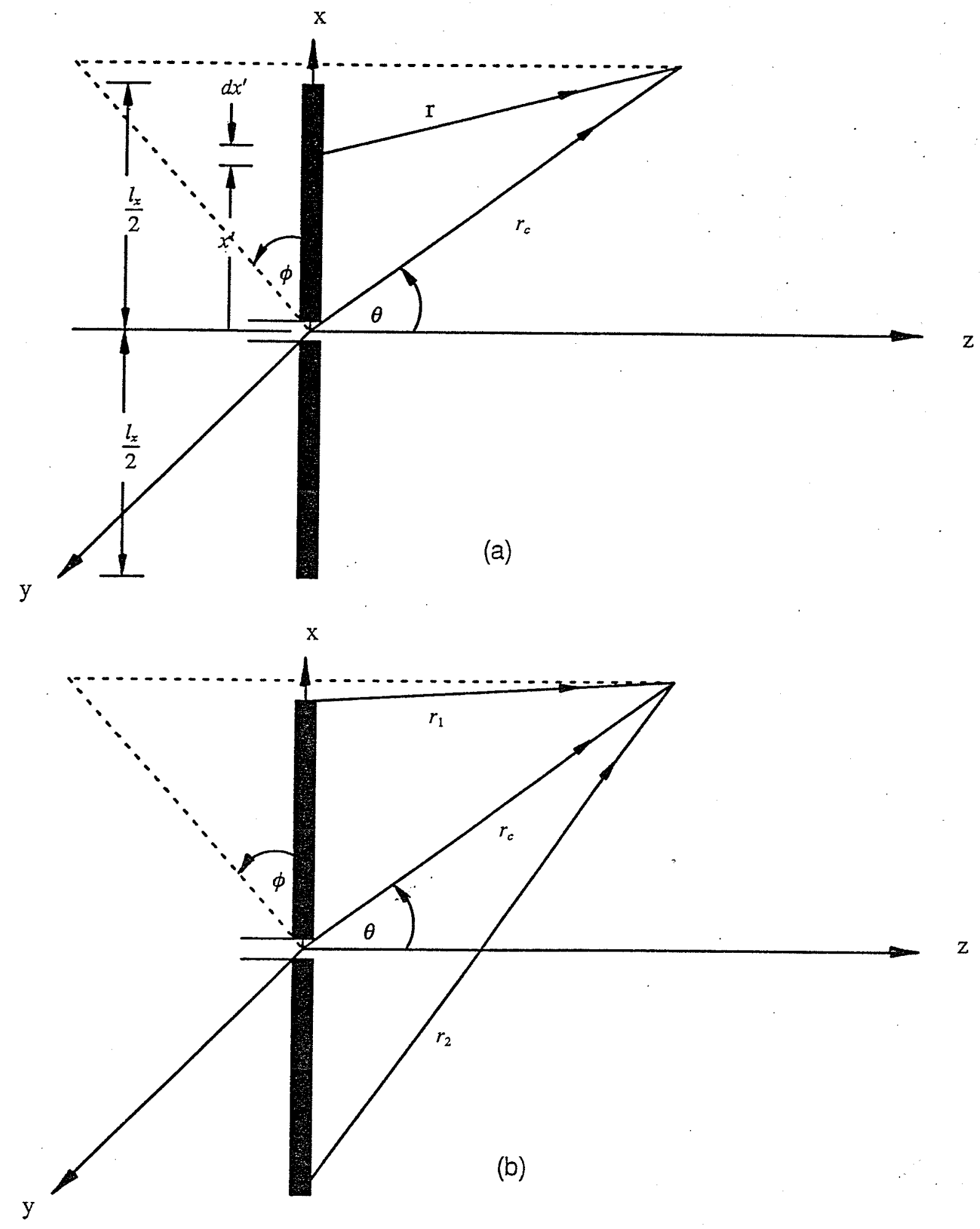

Fig. A.1 : Dipole geometry for near field analysis. 
where for $y^{\prime}=z^{\prime}=0$

$r=\left[\left(x-x^{\prime}\right)^{2}+y^{2}+z^{2}\right]^{\frac{1}{2}}$

The magnetic field intensity can be written in rectangular coordinates as ;

$$
\begin{aligned}
\mathbf{H} & =\frac{1}{\mu} \nabla \times \mathbf{A} \\
& =\frac{1}{\mu}\left[\frac{\partial A_{x}}{\partial z} \hat{a}_{y}-\frac{\partial A_{x}}{\partial y} \hat{a}_{z}\right]
\end{aligned}
$$

Using (A.3) into (A.4), one obtains the components of the magnetic field intensity as ;

$H_{x}=0$

$H_{y}=+\frac{I_{o}}{4 \pi} \frac{\partial U_{x}}{\partial z}$

$H_{z}=-\frac{I_{o}}{4 \pi} \frac{\partial U_{x}}{\partial y}$

where

$$
U_{x}=\int_{-\frac{l_{x}}{2}}^{0} \sin \left[k\left[\frac{l_{x}}{2}+x^{\prime}\right]\right] \frac{e^{-j k r}}{r} d x^{\prime}+\int_{0}^{+\frac{l_{x}}{2}} \sin \left[k\left(\frac{l_{x}}{2}-x^{\prime}\right]\right] \frac{e^{-j k r}}{r} d x^{\prime}
$$

Using Euler's relation

$\sin k\left(\frac{l_{x}}{2} \pm x^{\prime}\right)=\frac{e^{+j k\left(\frac{l_{x}}{2} \pm x^{\prime}\right)}-e^{-j k\left(\frac{l_{x}}{2} \pm x^{\prime}\right)}}{2 j}$

reduces $(\mathrm{A} .5 \mathrm{~d})$ to 


$$
\left.\begin{array}{r}
U_{x}=\frac{1}{2 j}\left\{+e^{+j k \frac{l_{x}}{2}} \int_{-\frac{l_{x}}{2}}^{0} \frac{e^{-j k\left(r-x^{\prime}\right)}}{r} d x^{\prime}-e^{-j k \frac{l_{x}}{2}} \int_{-\frac{l_{x}}{2}}^{0} \frac{e^{-j k\left(r+x^{\prime}\right)}}{r} d x^{\prime}\right. \\
+e^{+j k \frac{l_{x}}{2}} \int_{0}^{+\frac{l_{x}}{2}} \frac{e^{-j k\left(r+x^{\prime}\right)}}{r} d x^{\prime}-e^{-j k \frac{l_{x}}{2}} \int_{0}^{+\frac{l_{x}}{2}} \frac{e^{-j k\left(r-x^{\prime}\right)}}{r} d x^{\prime}
\end{array}\right\}
$$

Using (A.7) into (A.5b), we obtain ;

$$
\left.\begin{array}{rl}
H_{y}=+\frac{I_{o}}{8 \pi j}\left\{+e^{+j k \frac{l_{x}}{2}} \int_{-\frac{l_{x}}{2}}^{0} \frac{\partial}{\partial z}\left[\frac{e^{-j k\left(r-x^{\prime}\right)}}{r}\right] d x^{\prime}-e^{-j k \frac{l_{x}}{2}} \int_{-\frac{l_{x}}{2}}^{0} \frac{\partial}{\partial z}\left[\frac{e^{-j k\left(r+x^{\prime}\right)}}{r}\right] d x^{\prime}\right. \\
+e^{+j k \frac{l_{x}}{2}} \int_{0}^{+\frac{l_{x}}{2}} \frac{\partial}{\partial z}\left[\frac{e^{-j k\left(r+x^{\prime}\right)}}{r}\right] d x^{\prime}-e^{-j k \frac{l_{x}}{2}} \int_{0}^{+\frac{l_{x}}{2}} \frac{\partial}{\partial z}\left[\frac{e^{-j k\left(r-x^{\prime}\right)}}{r}\right] d x^{\prime}
\end{array}\right\}
$$

also using (A.7) into (A.5c), we obtain ;

$$
\left.\begin{array}{rl}
H_{z}=-\frac{I_{o}}{8 \pi j}\left\{+e^{+j k \frac{l_{x}}{2}} \int_{-\frac{l_{x}}{2}}^{0} \frac{\partial}{\partial y}\left[\frac{e^{-j k\left(r-x^{\prime}\right)}}{r}\right] d x^{\prime}-e^{-j k \frac{l_{x}}{2}} \int_{-\frac{l_{x}}{2}}^{0} \frac{\partial}{\partial y}\left[\frac{e^{-j k\left(r+x^{\prime}\right)}}{r}\right] d x^{\prime}\right. \\
+e^{+j k \frac{l_{x}}{2}} \int_{0}^{+\frac{l_{x}}{2}} \frac{\partial}{\partial y}\left[\frac{e^{-j k\left(r+x^{\prime}\right)}}{r}\right] d x^{\prime}-e^{-j k \frac{l_{x}}{2}} \int_{0}^{+\frac{l_{x}}{2}} \frac{\partial}{\partial y}\left[\frac{e^{-j k\left(r-x^{\prime}\right)}}{r}\right] d x^{\prime}
\end{array}\right\}
$$

$-88-$ 
The third term within the brackets of (A.8a) can expressed as ;

$$
e^{+j k \frac{l_{x}}{2}} \int_{0}^{+\frac{l_{x}}{2}} \frac{\partial}{\partial z}\left[\frac{e^{-j k\left(r+x^{\prime}\right)}}{r}\right] d x^{\prime}=z e^{+j k \frac{l_{x}}{2}} \int_{0}^{+\frac{l_{x}}{2}} e^{-j k\left(r+x^{\prime}\right)}\left[-\frac{1}{r^{3}}-j k \frac{1}{r^{2}}\right] d x^{\prime}
$$

Consider now the differential of

$d\left[\frac{e^{-j k\left(r+x^{\prime}\right)}}{r\left(r+x^{\prime}-x\right)}\right]=e^{-j k\left(r+x^{\prime}\right)}\left[-\frac{1}{r^{3}}-j k \frac{1}{r^{2}}\right] d x^{\prime}$

which is an exact differential. With the aid of (A.10), (A.9) can be integrated and expressed as

$$
\begin{aligned}
& e^{+j k \frac{l_{x}}{2}} \int_{0}^{+\frac{l_{x}}{2}} \frac{\partial}{\partial z}\left[\frac{e^{-j k\left(r+x^{\prime}\right)}}{r}\right] d x^{\prime}=+z e^{+j k \frac{l_{x}}{2}} \int_{0}^{+\frac{l_{x}}{2}} e^{-j k\left(r+x^{\prime}\right)}\left[-\frac{1}{r^{3}}-j k \frac{1}{r^{2}}\right] d x^{\prime} \\
& =+z e^{+j k \frac{l_{x}}{2}} \int_{0}^{+\frac{l_{x}}{2}} d\left[\frac{e^{-j k\left(r+x^{\prime}\right)}}{r\left(r+x^{\prime}-x\right)}\right] \\
& =+z e^{+j k \frac{l_{x}}{2}}\left[\frac{e^{-j k\left(r_{1}+\frac{l_{x}}{2}\right)}}{r_{1}\left(r_{1}+\frac{l_{x}}{2}-x\right)}\right]-z e^{+j k \frac{l_{x}}{2}}\left[\frac{e^{-j k r_{c}}}{r_{c}\left(r_{c}-x\right)}\right]
\end{aligned}
$$

where according to Fig. A.1b

$$
\begin{aligned}
& r_{1}=\left[\left(x-\frac{l_{x}}{2}\right)^{2}+y^{2}+z^{2}\right]^{\frac{1}{2}} \\
& r_{c}=\left(x^{2}+y^{2}+z^{2}\right)^{\frac{1}{2}}
\end{aligned}
$$

$-89-$ 


$$
r_{2}=\left[\left(x+\frac{l_{x}}{2}\right)^{2}+y^{2}+z^{2}\right]^{\frac{1}{2}}
$$

In a similar manner, the other terms of (A.8a) can be written as ;

$$
\begin{aligned}
& +e^{+j k \frac{l_{x}}{2}} \int_{-\frac{l_{x}}{2}}^{0} \frac{\partial}{\partial z}\left[\frac{e^{-j k\left(r-x^{\prime}\right)}}{r}\right] d x^{\prime}= \\
& -z e^{+j k \frac{l_{x}}{2}}\left[\frac{e^{-j k r_{c}}}{r_{c}\left(r_{c}+x\right)}\right]+z e^{+j k \frac{l_{x}}{2}}\left[\frac{e^{-j k\left(r_{2}+\frac{l_{x}}{2}\right)}}{r_{2}\left(r_{2}+\frac{l_{x}}{2}+x\right)}\right] \\
& -e^{-j k \frac{l_{x}}{2}} \int_{-\frac{l_{x}}{2}}^{0} \frac{\partial}{\partial z}\left[\frac{e^{-j k\left(r+x^{\prime}\right)}}{r}\right] d x^{\prime}= \\
& -z e^{-j k \frac{l_{x}}{2}}\left[\frac{e^{-j k r_{c}}}{r_{c}\left(r_{c}-x\right)}\right]+z e^{-j k \frac{l_{x}}{2}}\left[\frac{e^{-j k\left(r_{2}-\frac{l_{x}}{2}\right)}}{r_{2}\left(r_{2}-\frac{l_{x}}{2}-x\right)}\right] \\
& -e^{-j k \frac{l_{x}}{2}} \int_{0}^{+\frac{l_{x}}{2}} \frac{\partial}{\partial z}\left[\frac{e^{-j k\left(r-x^{\prime}\right)}}{r}\right] d x^{\prime}= \\
& +z e^{-j k \frac{l_{x}}{2}}\left[\frac{e^{-j k\left(r_{1}-\frac{l_{x}}{2}\right)}}{r_{1}\left(r_{1}-\frac{l_{x}}{2}+x\right)}\right]-z e^{-j k \frac{l_{x}}{2}}\left[\frac{e^{-j k r_{c}}}{r_{c}\left(r_{c}+x\right)}\right]
\end{aligned}
$$

$-90-$ 
Using (A.11)---(A.11f) reduces (A.8a) to ;

$H_{y}=+\frac{I_{o}}{4 \pi j} \frac{z}{\left(y^{2}+z^{2}\right)}\left[e^{-j k r_{1}}-2 \cos \left(k \frac{l_{x}}{2}\right) e^{-j k r_{c}}+e^{-j k r_{2}}\right]$

In a similar manner, (A.8b) can be written as ;

$$
H_{z}=-\frac{I_{o}}{4 \pi j} \frac{y}{\left(y^{2}+z^{2}\right)}\left[e^{-j k r_{1}}-2 \cos \left(k \frac{l_{x}}{2}\right) e^{-j k r_{c}}+e^{-j k r_{2}}\right]
$$




\section{Appendix B.}

\section{Near Fields of a Finite Dipole oriented along the z-axis and fed by a Sinusoidal Current Distribution}

Referring to Fig. B.1a and assuming a sinusoidal current distribution along the element given as ;

$$
\mathbf{I}_{e}\left(x^{\prime}, y^{\prime}, z^{\prime}\right)= \begin{cases}\hat{a}_{z} I_{o} \sin \left[k\left(\frac{l_{z}}{2}-z^{\prime}\right)\right] & 0 \leq z^{\prime} \leq+\frac{l_{z}}{2} \\ \hat{a}_{z} I_{o} \sin \left[k\left(\frac{l_{z}}{2}+z^{\prime}\right]\right] & -\frac{l_{z}}{2} \leq z^{\prime} \leq 0\end{cases}
$$

Following the same procedure as in Appendix A, we can write the components of the magnetic field intensity as ;

$$
\begin{aligned}
& H_{x}=+\frac{I_{o}}{4 \pi j} \frac{y}{\left(x^{2}+y^{2}\right)}\left[e^{-j k r_{1}}-2 \cos \left(k \frac{l_{z}}{2}\right) e^{-j k r_{c}}+e^{-j k r_{2}}\right] \\
& H_{y}=-\frac{I_{o}}{4 \pi j} \frac{x}{\left(x^{2}+y^{2}\right)}\left[e^{-j k r_{1}}-2 \cos \left(k \frac{l_{z}}{2}\right) e^{-j k r_{c}}+e^{-j k r_{2}}\right] \\
& H_{z}=0.0
\end{aligned}
$$

where according to Fig. B.1b

$$
\begin{aligned}
& r_{1}=\left[x^{2}+y^{2}+\left(z-\frac{l_{z}}{2}\right)^{2}\right]^{\frac{1}{2}} \\
& r_{c}=\left(x^{2}+y^{2}+z^{2}\right)^{\frac{1}{2}} \\
& r_{2}=\left[x^{2}+y^{2}+\left(z+\frac{l_{z}}{2}\right)^{2}\right]^{\frac{1}{2}}
\end{aligned}
$$



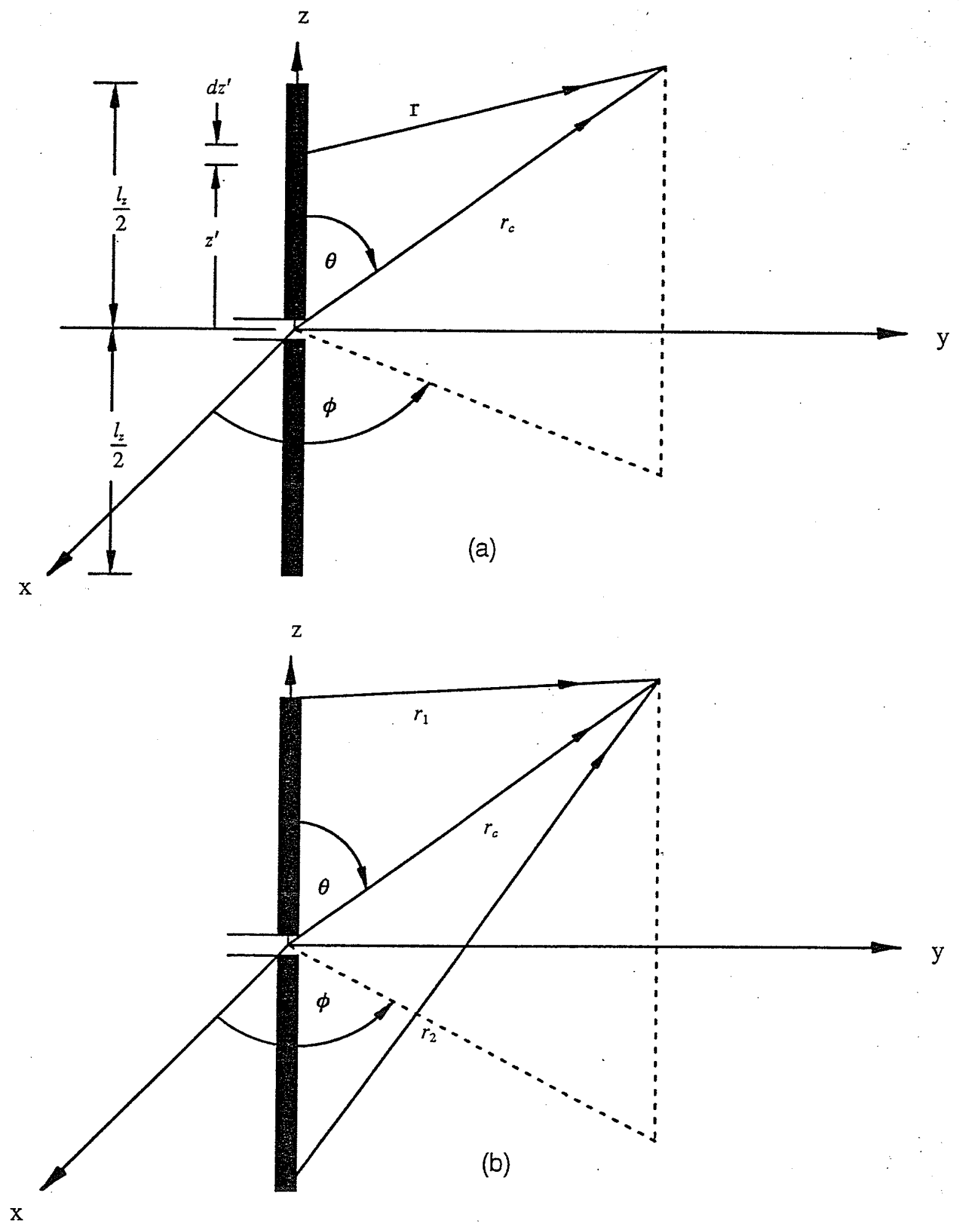

Fig. B.1 : Dipole geometry for near field analysis. 


\section{Appendix C. \\ Design and Analysis of Microstrip Comb Line \\ Linear Array Antenna \\ with known Excitation Distribution}

A computer aided design procedure is presented for a series-fed arrangement of microstrip comb line linear antenna in traveling wave mode. A microstrip comb line array is shown in Fig. C.1a. It is basically a traveling wave structure using a number of open-circuit terminations as radiating elements. Each of these terminations is connected to a common feeder line by a half wavelength resonant stub so that at resonance the radiation admittance is transformed directly on to the feeder line and Tjunctions are formed at the place where the stub is attached to the feeder.

Typically the design procedure of any array antenna commences by choosing the excitation current distribution and then calculating the element excitation coefficients according to the required specifications. Having calculated the excitation coefficients $A_{i}$ according to the focal region field [58], the second major step in the design process is to proceed in evaluating the electrical quantities, ending by obtaining the physical dimensions of the structure. It is evident that radiation conductances of the stubs give a measure of their radiated power. Thus, the stub radiation conductances are essentially proportional to the square of the excitation coefficients. For a traveling wave array, the exact calculation of the values of the radiation conductance $G_{r}$ to produce a required amplitude distribution, given the percentage power loss in the matched load, $P_{L}$, requires an n-variable optimization procedure and is involved if losses in the line or the susceptance loadings $B_{r}$ are significant. However, for long arrays, it is sufficiently accurate to perform a reverse iteration procedure starting at the load [59]. The radiation conductance of the ith element normalized to the line admittance $Y_{L}$, is 


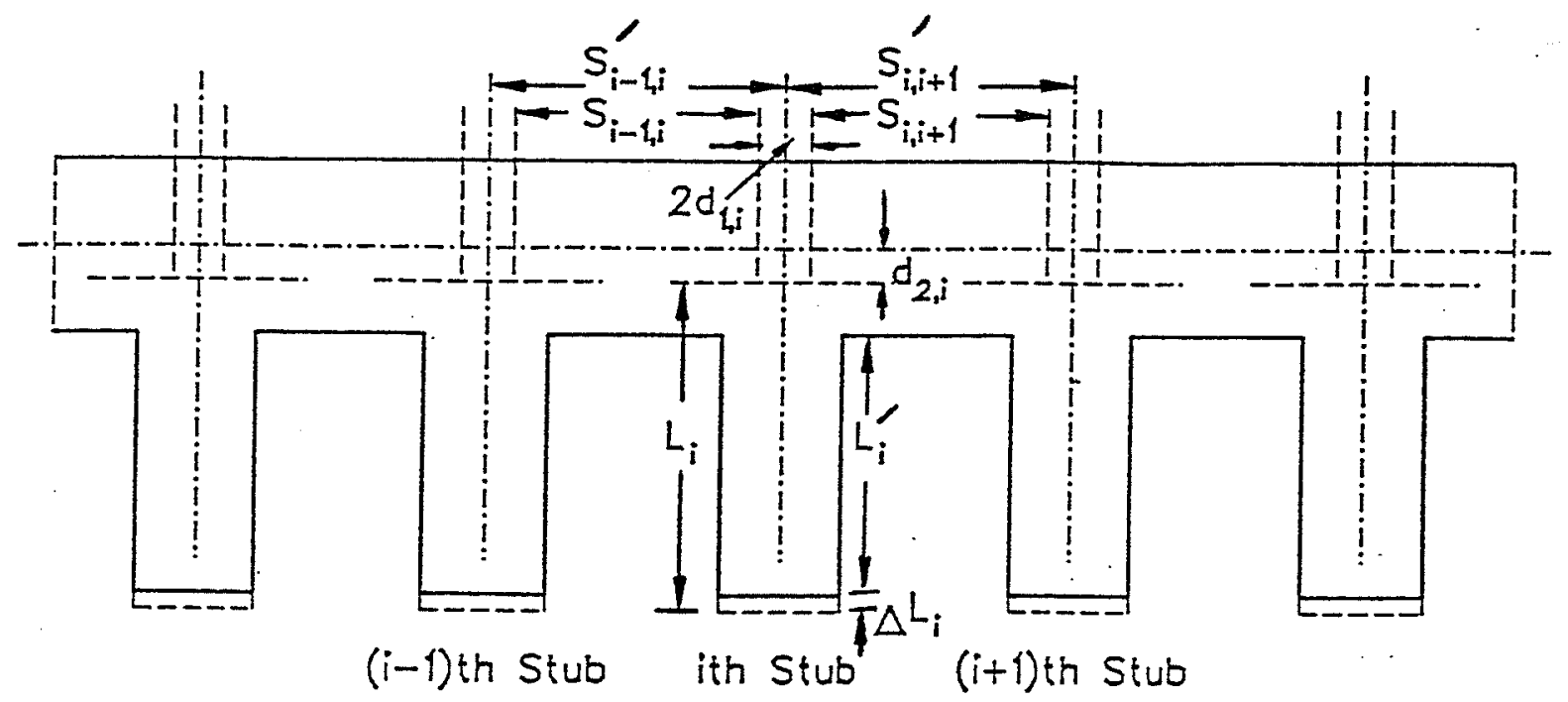

(a)

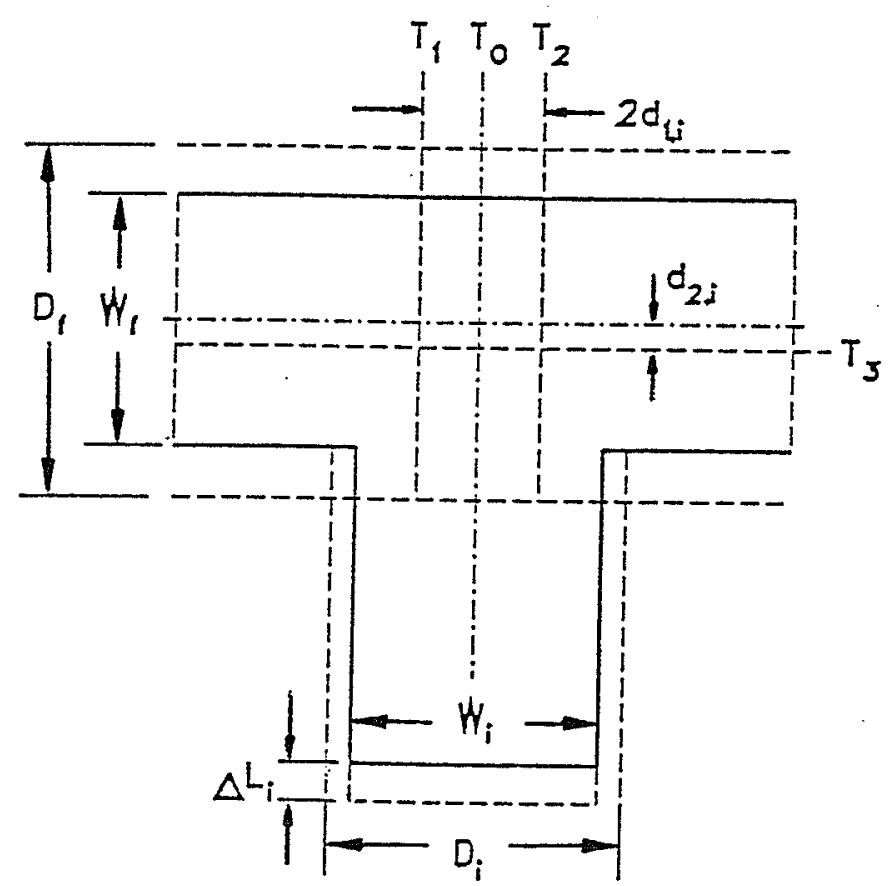

(b)

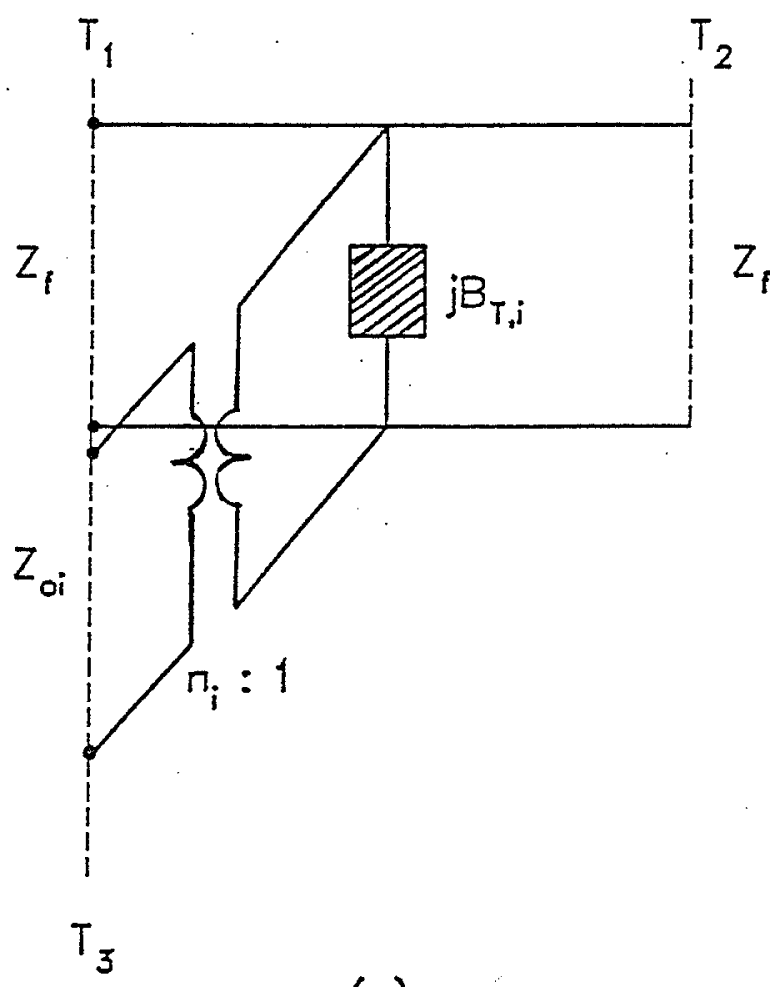

(c)

Fig. C.1 : Microstrip comb line array

(a) Array element geometry showing the ith stub (b\&c) T-junction and its equivalent circuit 


$$
G_{r, i}=P_{i}\left\{P_{L}+\sum_{v=1}^{v=n} P_{v}\right\}^{-1}
$$

where $P_{i}$ is the power radiated by the ith stub and the array has $\mathrm{n}$ stubs.

These normalized conductance values are transformed to actual values by considering the specified impedance level of system $Z_{f}$ typically used as ( $50 \mathrm{ohm}$ ).

On the other hand, the radiation conductances of an open-circuit microstrip termination is to a good approximation, a simple function of the strip width, approximated to $\frac{h}{\lambda}$ and $\frac{D_{i}}{\lambda} \ll 1$ to give the actual radiation conductance of the ith stub termination as [69] ;

$$
G_{r, i}=\frac{4 \pi}{3 Z_{o i}} \frac{h}{\lambda} \frac{D_{i}}{\lambda} \frac{1}{\sqrt{\varepsilon_{e i}}}
$$

where $D_{i}, Z_{o i}$ and $\varepsilon_{e i}$ are the effective width, characteristic impedance and the effective dielectric constant of the ith stub respectively, and $h$ is the substrate thickness.

Basically, a comb line array uses half guide wavelength long microstrip radiators open circuited at only one end, with the remaining ends excited directly by a feed transmission line with one guide wavelength separation between consecutive stubs. However, accurate or almost accurate implementation of such a microstrip design requires the reduction of a cut and try process. This can be achieved easily by including effects of microstrip discontinuities in the design process.

Generally, the discontinuities are represented by an equivalent circuit, defined between reference planes at which semi-infinite lines terminate. In the comb line structure, T-junctions are formed at places where stubs are attached to the feeder line. Fig. C.1(b \& c) show such a junction and its equivalent circuit. The modeling of the Tjunction and its corresponding relationships are based upon [65]. The effective width of the feeder line $D_{f}$ and of the ith stub $D_{i}$ are expressed by ; 


$$
\begin{aligned}
& D_{f}=\frac{120 \pi h}{Z_{f} \sqrt{\varepsilon_{e f}}} \\
& \frac{D_{i}}{\lambda}=120 \pi \frac{h}{\lambda} \frac{1}{Z_{o i} \sqrt{\varepsilon_{e i}}}
\end{aligned}
$$

and the transformer turns ratio of the ith stub is ;

$$
n_{i}=\frac{\sin \left(\frac{\pi}{2} \frac{2 D_{f}}{\lambda_{g f}} \frac{Z_{f}}{Z_{o i}}\right)}{\frac{\pi}{2} \frac{2 D_{f}}{\lambda_{g f}} \frac{Z_{f}}{Z_{o i}}}
$$

where $Z_{f}, \varepsilon_{e f}$ and $\lambda_{g f}$ are the characteristic impedance, effective dielectric constant and guide wavelength of the feeder line, respectively.

Substitute for $\frac{D_{i}}{\lambda}$ from (C.3b) in (C.2), we end with ;

$Z_{o i} \sqrt{\varepsilon_{e i}}=4 \pi \frac{h}{\lambda}\left[\frac{10}{G_{r, i}}\right]^{\frac{1}{2}}$

The characteristic impedance and the effective dielectric constant of the ith stub are given by $[66,68]$ as ;

$$
\begin{array}{ll}
Z_{o i}=\frac{Z_{o}}{2 \pi \sqrt{\varepsilon_{e i}}} \ln \left[8 \frac{h}{w_{i}}+0.25 \frac{w_{i}}{h}\right] & \text { for } \frac{w_{i}}{h} \ll 1 \\
Z_{o i}=\frac{Z_{o}}{\sqrt{\varepsilon_{e i}}}\left[\frac{w_{i}}{h}+1.393+0.667 \ln \left(\frac{w_{i}}{h}+1.414\right)\right]^{-1} & \text { for } \frac{w_{i}}{h} \gg 1
\end{array}
$$

where $Z_{o}=120 \pi$

$$
\begin{array}{ll}
\varepsilon_{e i}=\frac{\varepsilon_{r}+1}{2}+\frac{\varepsilon_{r}-1}{2} F_{1}\left(\frac{w_{i}}{h}\right) & \text { for } \frac{w_{i}}{h} \ll 1 \\
F_{1}\left(\frac{w_{i}}{h}\right)=\left(1+12 \frac{h}{w_{i}}\right)^{-\frac{1}{2}}+0.04\left(1-\frac{w_{i}}{h}\right) 2 &
\end{array}
$$




$$
\begin{array}{ll}
\varepsilon_{e i}=\frac{\varepsilon_{r}+1}{2}+\frac{\varepsilon_{r}-1}{2} F_{2}\left(\frac{w_{i}}{h}\right) & \text { for } \frac{w_{i}}{h}>>1 \\
F_{2}\left(\frac{w_{i}}{h}\right)=\left(1+12 \frac{h}{w_{i}}\right)^{-\frac{1}{2}} &
\end{array}
$$

using (C.5a).....(C.5e) into (C.4), one can calculate the effective characteristic impedance $Z_{o i}$, the effective dielectric constant $\varepsilon_{e i}$ and the effective width $w_{i}$ of the ith stub.

The guide wavelength of the feeder line $\lambda_{g f}$ can be obtained from [68] as ;

$$
\lambda_{g f}=\frac{\lambda}{\sqrt{\varepsilon_{e f}}}
$$

where $\lambda$ is the free space wavelength for a given operating frequency $f_{o}$ and $\varepsilon_{e f}$ is given as ;

$\varepsilon_{e f}=\varepsilon_{r}-\frac{\varepsilon_{r}-\varepsilon_{e}}{1+G(f o / f p) 2}$

$G=\left(\frac{Z_{o}-5}{Z_{o}}\right) 2+0.004 Z_{o}$

$f_{p}=\frac{Z_{o}}{2 \mu_{o} h}$

The equations for the displacement of the reference planes as shown in Fig. C.1a are ;

$$
\frac{d_{1, i}}{D_{i}}=0.05 \frac{Z_{f}}{Z_{o i}} n_{i}^{2}
$$

and

$$
\frac{d_{1, i}}{D_{f}}=\frac{1}{2}-0.16\left[1+\left(\frac{2 D_{f}}{\lambda_{g f}}\right)^{2}-2 \ln \left(\frac{Z_{f}}{Z_{o i}}\right)\right] \frac{Z_{f}}{Z_{o i}} \quad \text { for } \quad \frac{Z_{f}}{Z_{o i}} \leq 2
$$

or

$$
\frac{d_{2, i}}{D_{f}}=\frac{1}{2}-0.16\left[1+\left(\frac{2 D_{f}}{\lambda_{g f}}\right)^{2}-2 \ln \left(\frac{Z_{f}}{Z_{o i}}\right)\right] \frac{Z_{o i}}{Z_{f}} \quad \text { for } \quad \frac{Z_{f}}{Z_{o i}} \geq 2
$$


The shunt susceptance of the ith stub $B_{T, i}$ is given by:

$$
B_{T, i}=\frac{D_{f}}{Z_{f} \lambda_{g f}}\left[-\left(1-\frac{2 D_{f}}{\lambda_{g f}}\right)\right] \frac{Z_{f}}{Z_{o i}} \quad \text { for } \frac{Z_{f}}{Z_{o i}} \leq 0.5
$$

or

$$
B_{T, i}=\frac{D_{f}}{Z_{f} \lambda_{g f}}\left[+\left(1-\frac{2 D_{f}}{\lambda_{g f}}\right)\left(3 \frac{Z_{f}}{Z_{o i}}-2\right)\right] \quad \text { for } \frac{Z_{f}}{Z_{o i}} \geq 0.5
$$

The other discontinuities in the comb line structure are the open-end of the stubs. Such a discontinuity is represented by an excess capacitance which can be transformed into an equivalent length of transmission line $\left(\Delta L_{i}\right)$ as shown in Fig. C.1b. An empirical expression for $\left(\frac{\Delta L_{i}}{h}\right)$ is appeared in [66] and used here as ;

$$
\frac{\Delta L_{i}}{h}=0.412 \cdot\left\{\frac{\left(\varepsilon_{e i}+0.300\right)\left(\frac{w_{i}}{h}+0.264\right)}{\left(\varepsilon_{e i}-0.258\right)\left(\frac{w_{i}}{h}+0.800\right)}\right\}
$$

The correction factors resulting from discontinuity considerations $\left(d_{1, i}\right)$, $\left(d_{2, i}\right)$ and $\Delta L_{i}$ modify the ith stub physical length $\left(L^{\prime}{ }_{i}\right)$ and the inter stub separation $\left(S^{\prime}{ }_{i-1, i}\right)$ into the electrical (effective) length $\left(L_{i}\right)$ and separation $\left(S_{i-1, i}\right)$ respectively as expressed in the following relationships :

$$
L^{\prime}{ }_{i}=L_{i}-\Delta L_{i}-\left(\frac{W_{f}}{2}-d_{2, i}\right)
$$

and

$$
S_{i-1, i}^{\prime}=S_{i-1, i}+d_{1, i-1}+d_{1, i}
$$

A traveling wave antenna is a structure having the properties of conveying energy by guiding waves at the same time causing a coherent radiation into space. The radiating elements of the array are disposed along a transmission line which is excited at one end and each of its elements couples a portion of the energy to the next one. After the last element a remaining small portion of power is left to be dissipated in a match 
load terminating the array at the other end. Input admittance (impedance) of the array is an important parameter in the design process. To calculate the input admittance for a traveling wave array, consider the equivalent circuit for a typical stub and its associated T-junction shown in Fig. C.2a. Since the stub is essentially a half wavelength long, hence its radiating admittance $\left(G_{r, i}+j B_{r, i}\right)$ can be transformed directly onto the feeder line after multiplying by the $\left(n_{i}{ }^{2}\right)$. Resulting in a parallel combination with the shunt susceptance $\left(j B_{T, i}\right)$ of the junction, and a total admittance $\left(n_{i}^{2}\left[G_{r, i}+j B_{r, i}\right]+j B_{T, i}\right)$. To cancel out this imaginary part i.e. the total admittance should be $\left(n_{i}{ }^{2} G_{r, i}\right)$, the electrical length of the stub is reduced in order to force $j n_{i}^{2} B_{r, i}=-j B_{T, i}$. The new electrical length of the stub should be $\left(\frac{q \lambda_{g i}}{2}\right)$, where $\mathrm{q}$ is a constant less than unity. By using the principles of transmission line theory, the total admittance $\left(Y_{r, i}\right)$ at the ith node due to the ith stub and its associated junction may be written as ;

$$
Y_{r, i}=j B_{T, i}+n_{i}^{2}\left[Y_{o, i} \frac{G_{r, i}+j\left(B_{r, i}+Y_{o, i} \beta_{i}\right)}{\left(Y_{o, i}-B_{r, i} \beta_{i}\right)+j G_{r, i} \beta_{i}}\right]
$$

where $\left(Y_{o, i}\right)$ and $\beta_{i}=\frac{2 \pi}{\lambda_{g i}}$ are the ith stub characteristic admittance and wave number respectively. By equating the imaginary part in the right hand side of Eqn. (C.12) to zero, we have :

$$
\tan ^{2} \beta_{i} L_{i}+b b \tan \beta_{i} L_{i}+c c=0
$$

where

$$
b b=Y_{o, i}\left[\frac{Y_{o, i}-\left(G_{r, i}^{2}+B_{r, i}^{2}\right)-\frac{B_{T, i}}{n_{i}^{2}} B_{r, i} B_{T, i}}{\frac{B_{T, i}}{n_{i}^{2}}\left(G_{r, i}^{2}+B_{r, i}^{2}\right)-Y_{o, i}^{2} B_{r, i}}\right]
$$



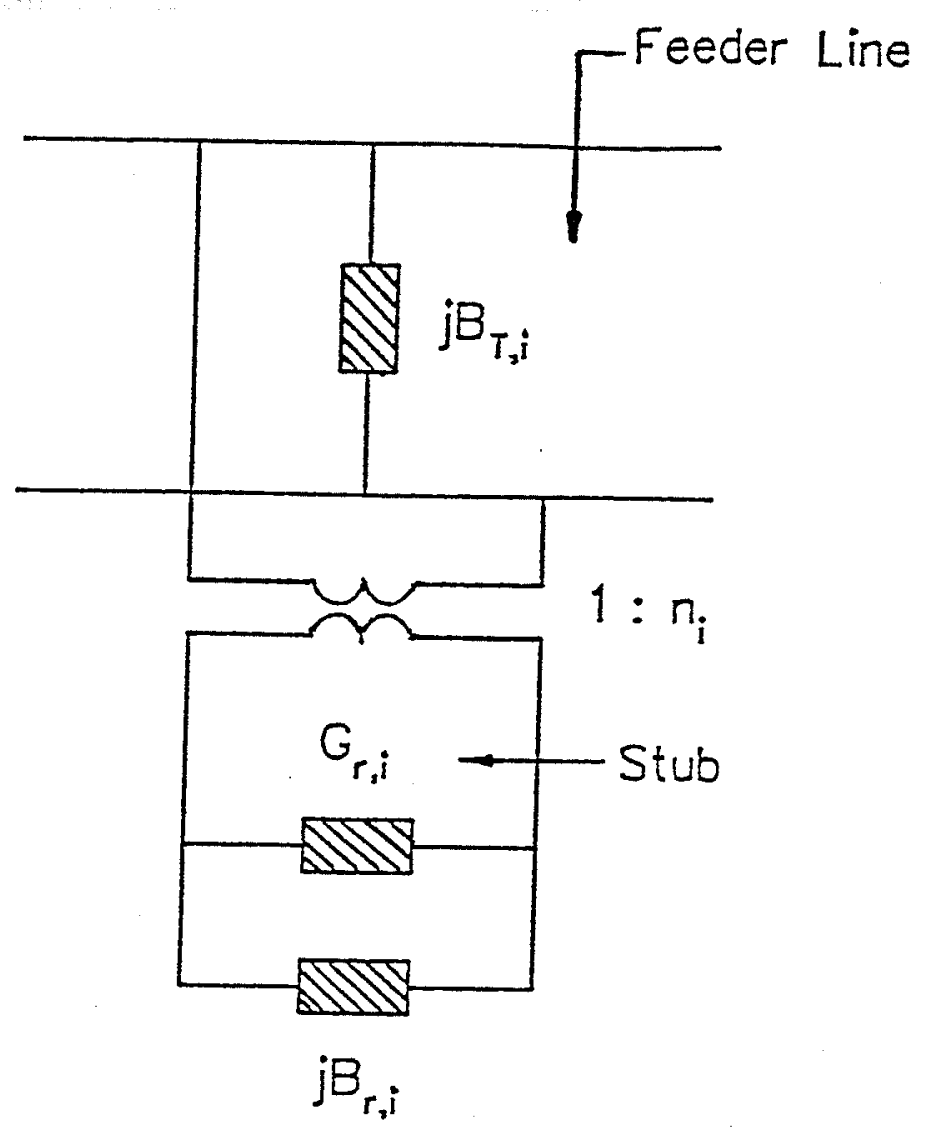

(a)

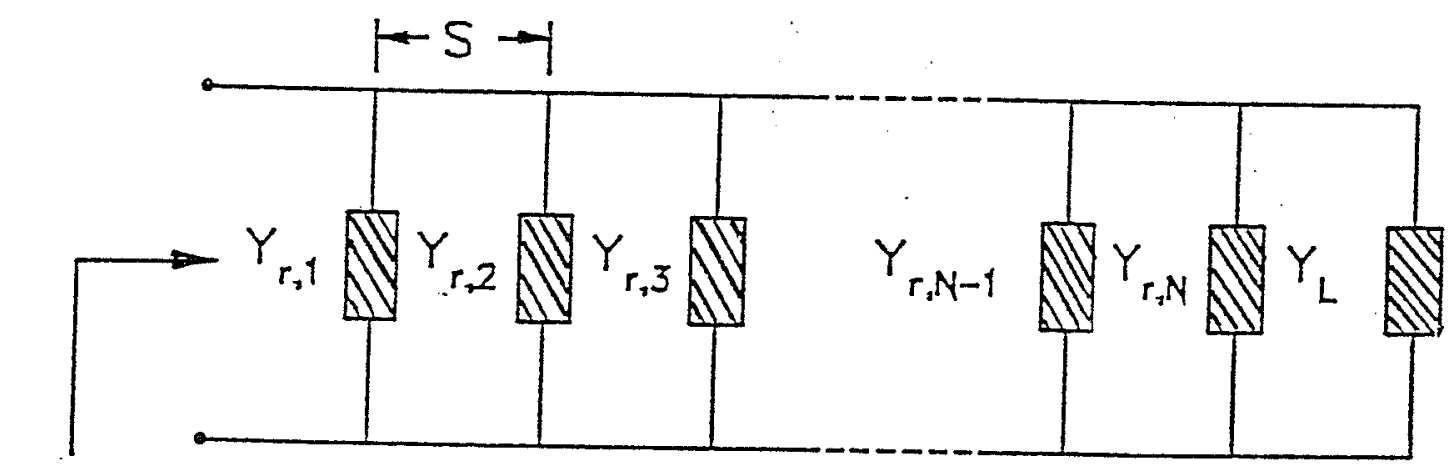

Input

$Y_{r, i}=$ ith element radiation admittance

(b)

Fig. C.2 : (a) The equivalent circuit for a typical stub and its associate $\mathrm{T}$-junction, (b) The traveling wave array with a resistive matched load $G_{1}$. 


$$
c c=\frac{Y_{o, i}^{2}\left(B_{r, i}+\frac{B_{T, i}}{{n_{i}}^{2}}\right)}{\frac{B_{T, i}}{n_{i}{ }^{2}}\left(G_{r, i}^{2}+B_{r, i}^{2}\right)-Y_{o, i}^{2} B_{r, i}}
$$

As is clearly shown, Eqn. (C.13a) is a second order equation in $\left(\tan \beta_{i} L_{i}\right)$, solving for $\left(\tan \beta_{i} L_{i}\right)$ and choosing the principle value of $\left(\beta_{i}\right)$ the electrical length $\left(L_{i}\right)$ which cancels the imaginary part of the ith stub input admittance can be calculated. The traveling wave array with a resistive matched load $\left(G_{l}\right)$ at the end is shown in Fig. C.2b. The overall input admittance of the array $\left(Y_{i n}\right)$ may be calculated by an iteration process starting at the Nth stub as ;

$$
Y_{i n}=G_{l}+\sum_{i=N}^{2}\left\{Y_{r, i-1}+Y_{f} \frac{Y_{r, i}+j Y_{f} \tan \beta_{f} S_{i}}{Y_{f}+j Y_{r, i} \tan \beta_{f} S_{i}}\right\}
$$

where $\left(Y_{f}\right)$ and $\beta_{f}=\frac{2 \pi}{\lambda_{g f}}$ are the characteristic admittance and wave number in the feeder line, respectively. 


\section{Appendix D. \\ Radiation Pattern of Open-Circuit Rectangular Microstrip Patch Resonator}

A conventional microstrip line of length $2 l$, conductor strip width $w$, substrate thickness $h$, substrate relative permittivity and permeability of $\varepsilon_{r}>1$ and $\mu_{r}=1$, respectively, lies in the $x z$-plane as sketched in Fig. D.1. To simplify the calculation the microstrip is offset to one side of the origin o, so that the conductor strip, of negligible thickness, lies in the $x z$-plane at $y=2 h$ : similarly, the conducting ground plane and the image of the conductor strip in the ground plane also lie in the $x z$-plane but at $y=h$ and 0 , respectively. The ground plane and substrate extend along the $\pm z-$ direction and have an overall widths $w_{s}$, but at $x= \pm l$ the microstrip structure is opencircuit and radiating apertures 1 and 2 lie in $y z$-plane at $x= \pm l$ where the substrate terminates. A good approximation to the waves guided by the microstrip is the transverse electromagnetic wave [67] (TEM) which is assumed to be concentrated under the strip and incident on each aperture where some power is radiated and the remainder reflected as a guided wave. Under these simplifying assumptions, the transverse field $\left(\mathbf{E}_{a}, \mathbf{H}_{a}\right)$ in aperture 1, which is now restricted to the rectangular region bounded by the conducting strip at $y=2 h$ and its image in the ground-plane at $y=0$, is given by

$$
\begin{aligned}
& \mathbf{E}_{a}=+\hat{a}_{y} E_{y} \exp (-j \beta l)(1+\bar{\Gamma}) \\
& \mathbf{H}_{a}=+\hat{a}_{z} H_{z} \exp (-j \beta l)(1-\bar{\Gamma}) \\
& E_{y}=Z H_{z}, Z=Z_{o} / \beta, \beta=k\left(\varepsilon_{e f f}\right)^{1 / 2}=2 \pi / \lambda_{g}
\end{aligned}
$$

where $\varepsilon_{e f f}$ is the effective permittivity of the microstrip substrate and a harmonic time variation of $\exp (+j \omega t)$ is assumed and omitted throughout. The complex reflection coefficient $\bar{\Gamma}=|\bar{\Gamma}| \exp \left(-j 2 \beta l^{\prime}\right)$ where $l^{\prime}$ is the end-effect, manifesting itself as a capacitive loading across the end of the open-circuit termination.

The far-field $\left(\mathbf{E}_{r a d}, \mathbf{H}_{\text {rad }}\right)$ corresponding to a field distribution $\left(\mathbf{E}_{a}, \mathbf{H}_{a}\right)$ in an aperture 


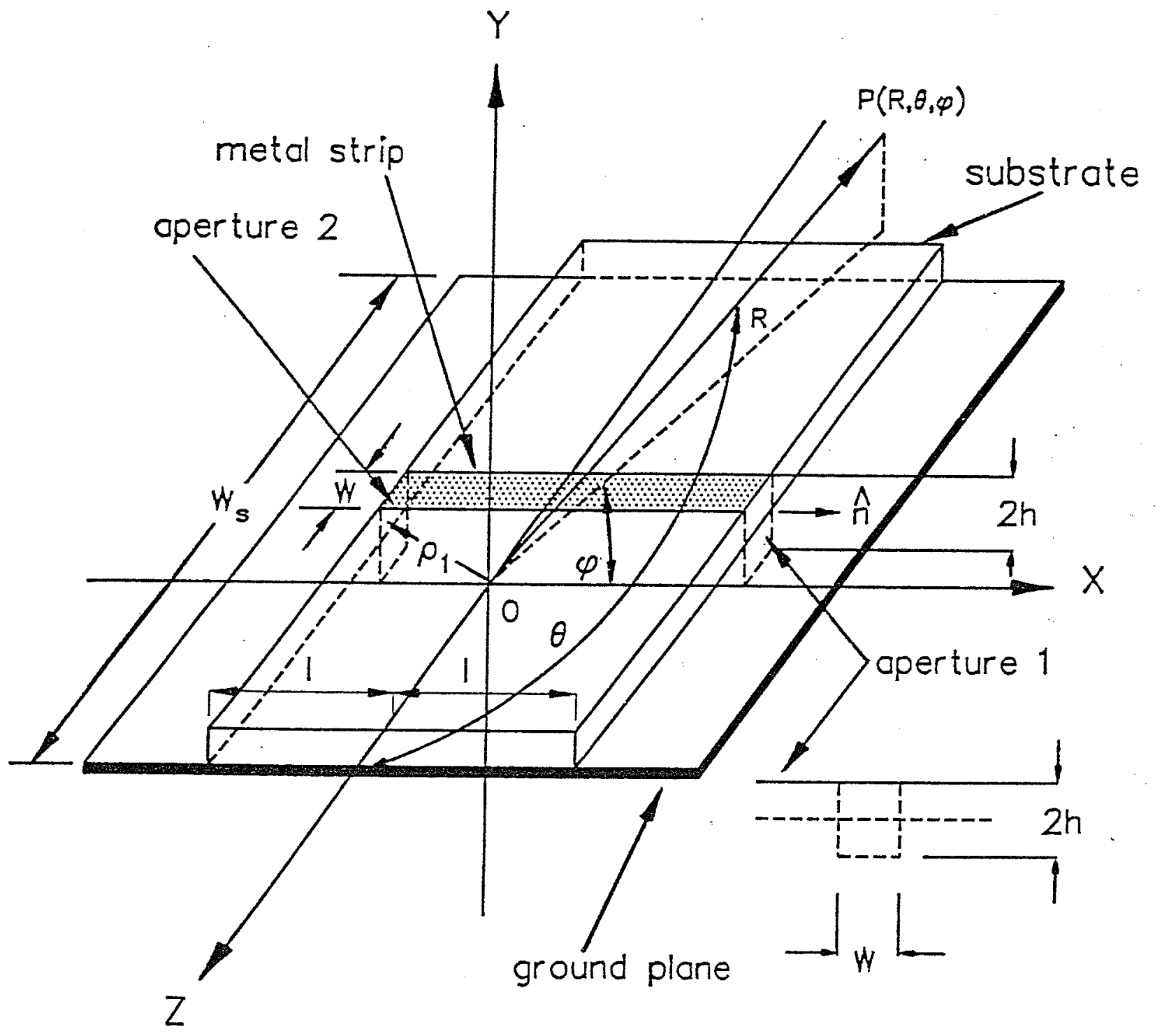

Fig. D.1 Sketch of microstrip line showing coordinate system and rectangular apertures at $x= \pm 1,0<<y<<2 h, 0<<z<<w$ 
$S_{a}$ is given by the far-field form of the vector Kirchoff formula as ;

$$
\begin{aligned}
& \mathbf{E}_{r a d}=K \hat{R}_{1} \times \int_{S_{a}}\left[\left(\hat{n} \times \mathbf{E}_{a}\right)-Z_{o} \hat{R}_{1} \times\left(\hat{n} \times \mathbf{H}_{a}\right)\right] \exp \left(j k \rho_{1} \cdot \hat{R}_{1}\right) d a \\
& \mathbf{H}_{\text {rad }}=K \hat{R}_{1} \times \int_{S_{a}}\left[\left(\hat{n} \times \mathbf{H}_{a}\right)+Z_{o} \hat{R}_{1} \times\left(\hat{n} \times \mathbf{E}_{a}\right)\right] \exp \left(j k \rho_{1} \cdot \hat{R}_{1}\right) d a
\end{aligned}
$$

where the vector quantities follow [48] notation and are defined in Fig. D.1 together with the co-ordinate system and $K=\frac{-j k}{4 \pi R} \exp (-j k R)$.

On substituting for ( $\left.\mathbf{E}_{a}, \mathbf{H}_{a}\right)$ from (D.1) in (D.2) and carrying out the appropriate transformation of unit vectors, one can write the radiation far field as ;

$$
\begin{aligned}
\mathbf{E}_{\text {rad }}=K \hat{R}_{1} \times \int_{S_{a}}-E_{y} \exp (-j \beta l)\left[-\hat{a}_{x} \cos \theta k\left(\varepsilon_{e f f}\right)^{1 / 2}(1-\bar{\Gamma})-\hat{a}_{z}[+(1+\bar{\Gamma})\right. \\
\left.\left.\quad-\sin \theta \cos \phi k\left(\varepsilon_{e f f}\right)^{1 / 2}(1-\bar{\Gamma})\right]\right] \exp \left(j k \rho_{1} \cdot \hat{R}_{1}\right) d a
\end{aligned}
$$

$$
\begin{gathered}
\mathbf{H}_{r a d}=\frac{1}{Z_{o}} K \hat{R}_{1} \times \int_{S_{a}}-E_{y} \exp (-j \beta l)\left[\hat{a}_{x} \sin \theta \sin \phi(1+\bar{\Gamma})+\hat{a}_{y}[+(1+\bar{\Gamma})\right. \\
\left.\left.\quad \sin \theta \cos \phi+k\left(\varepsilon_{e f f}\right)^{1 / 2}(1-\bar{\Gamma})\right]\right] \exp \left(j k \rho_{1} \cdot \hat{R}_{1}\right) d a
\end{gathered}
$$

Now $\rho_{1}=x^{\prime} \hat{a}_{x}+y^{\prime} \hat{a}_{y}+z^{\prime} \hat{a}_{z}$ is a vector from the origin $o$ to a point $\left(x^{\prime}, y^{\prime}, z^{\prime}\right)$ in the aperture and $\hat{R}_{1}=\sin \theta \cos \phi \hat{a}_{x}+\sin \theta \sin \phi \hat{a}_{y}+\cos \theta \hat{a}_{z}$; thus, eqn. (D.3) simplifies to ;

$$
\begin{aligned}
\mathbf{E}_{r a d}=\exp (j k \sin \theta \cos \phi-\beta) l \hat{R}_{1} \times & {\left[\hat{a}_{x} \cos \theta k\left(\varepsilon_{e f f}\right)^{1 / 2}(1-\bar{\Gamma})-\hat{a}_{z}[+(1+\bar{\Gamma})\right.} \\
& \left.\left.-\sin \theta \cos \phi k\left(\varepsilon_{e f f}\right)^{1 / 2}(1-\bar{\Gamma})\right]\right] I(\theta, \phi)
\end{aligned}
$$




$$
\begin{array}{r}
\mathbf{H}_{r a d}=\frac{1}{Z_{o}} \exp (j k \sin \theta \cos \phi-\beta) l \hat{R}_{1} \times\left[\hat{a}_{x} \sin \theta \sin \phi(1+\bar{\Gamma})+\hat{a}_{y}[+(1+\bar{\Gamma})\right. \\
\left.\left.\sin \theta \cos \phi+k\left(\varepsilon_{e f f}\right)^{1 / 2}(1-\bar{\Gamma})\right]\right] I(\theta, \phi)
\end{array}
$$

$$
\begin{aligned}
& I(\theta, \phi)=-E_{y} K \int_{y^{\prime}=0}^{y^{\prime}=2 h} \int_{z^{\prime}=0}^{z^{\prime}=w} \exp \left[j k\left(y^{\prime} \sin \theta \sin \phi+z^{\prime} \cos \theta\right)\right] d y^{\prime} d z^{\prime} \\
& \quad=-E_{y} K 2 h w \frac{\sin Y}{Y} \frac{\sin Z}{Z} \Psi \\
& Y=k h \sin \theta \sin \phi \\
& Z=\frac{1}{2} k w \cos \theta \\
& \Psi=\exp \left[j k\left(h \sin \theta \sin \phi+\frac{1}{2} w \cos \theta\right)\right]
\end{aligned}
$$

On transforming the unit Cartesian co-ordinates the far-field radiation terms corresponding to aperture 1 can be written as ;

$$
\begin{gathered}
E_{\theta I}=I(\theta, \phi)\left\{k\left(\varepsilon_{e f f}\right)^{1 / 2}[\exp (+j \zeta)-\bar{\Gamma} \exp (+j \xi)] \cdot(+\sin \theta \cos \phi)\right\} \\
E_{\phi I}=I(\theta, \phi)\{[\exp (+j \zeta)+\bar{\Gamma} \exp (+j \xi)] \cdot(+\sin \theta)+ \\
\left.k\left(\varepsilon_{e f f}\right)^{1 / 2}[\exp (+j \zeta)-\bar{\Gamma} \exp (+j \xi)] \cdot(+\cos \phi)\right\} \\
\mathbf{H}_{I}=I(\theta, \phi)\left\{[ \operatorname { e x p } ( + j \zeta ) + \overline { \Gamma } \operatorname { e x p } ( + j \xi ) ] \cdot \left[\hat{a}_{x}(-\sin \theta \cos \theta \cos \phi)+\right.\right. \\
\left.\hat{a}_{y}(-\sin \theta \cos \theta \sin \phi)+\hat{a}_{z}\left(+\sin ^{2} \theta\right)\right]+k\left(\varepsilon_{e f f}\right)^{1 / 2}[\exp (+j \zeta)-\bar{\Gamma} \exp (+j \xi)] \\
{\left[\left(\hat{a}_{x}(-\cos \theta)+\hat{a}_{z}(+\sin \theta \cos \phi)\right]\right\} \cdot \frac{1}{Z_{o}}} \\
\zeta=k\left[\sin \theta \cos \phi-\left(\varepsilon_{e f f}\right)^{1 / 2}\right] l, \xi=k\left[\sin \theta \cos \phi-\left(\varepsilon_{e f f}\right)^{1 / 2}\right] l-2 \beta l^{\prime}
\end{gathered}
$$


Consider aperture 2 in Fig. D.1 situated at $x=-l$; the aperture fields corresponding to eqn. (D.1), carrying the same assumptions and compatible with resonator action, are

$$
\begin{array}{r}
\mathbf{E}_{a}=+\hat{a}_{y} E_{y} \exp (+j \beta l)(1+\bar{\Gamma}) \\
\mathbf{H}_{a}=-\hat{a}_{z} H_{z} \exp (+j \beta l)(1-\bar{\Gamma})
\end{array}
$$

and on repeating the calculation of eqn. (D.6), based on eqn. (D.2) with $\hat{n}=-\hat{a}_{x}$, the radiation far-fields of aperture 2 can be written as ;

$$
\begin{aligned}
& E_{\theta I I}=I(\theta, \phi)\left\{k\left(\varepsilon_{e f f}\right)^{1 / 2}[\exp (-j \zeta)-\bar{\Gamma} \exp (-j \xi)] \cdot(+\sin \theta \cos \phi)\right\} \\
& E_{\phi I I}=I(\theta, \phi)\{[\exp (-j \zeta)+\bar{\Gamma} \exp (-j \xi)] \cdot(-\sin \theta)+ \\
& \left.k\left(\varepsilon_{e f f}\right)^{1 / 2}[\exp (-j \zeta)-\bar{\Gamma} \exp (-j \xi)] \cdot(+\cos \phi)\right\} \\
& \mathbf{H}_{I I}=I(\theta, \phi)\left\{[ \operatorname { e x p } ( - j \zeta ) + \overline { \Gamma } \operatorname { e x p } ( - j \xi ) ] \cdot \left[\hat{a}_{x}(+\sin \theta \cos \theta \cos \phi)+\right.\right. \\
& \left.\hat{a}_{y}(+\sin \theta \cos \theta \sin \phi)+\hat{a}_{z}\left(-\sin ^{2} \theta\right)\right]+k\left(\varepsilon_{e f f}\right)^{1 / 2}[\exp (-j \zeta)-\bar{\Gamma} \exp (-j \xi)] \cdot \\
& {\left[\left(\hat{a}_{x}(-\cos \theta)+\hat{a}_{z}(+\sin \theta \cos \phi)\right]\right\} \cdot \frac{1}{Z_{o}}}
\end{aligned}
$$

with $I(\theta, \phi), \zeta$ and $\xi$ as defined for eqn. (D.5). The assumed resonator action is that the wave reflected from aperture 1 is the incident wave approaching aperture 2 , and vice versa, with energy losses due to aperture radiation replenished by the feeder which is attached to some point on the resonator, with impedance properties compatible with feed system. Under these assumptions, and neglecting radiation losses from the feed point, losses in the substrate and radiation due surface irregularities and metal inhomogeneity along the resonator length, the combined radiation far-fields of aperture 1 and 2 are obtained by adding eqns. (D.5) and (D.7) thus ; 


$$
\begin{aligned}
& E_{\theta I+I I}= 2 I(\theta, \phi)\left\{k\left(\varepsilon_{e f f}\right)^{1 / 2}[\cos \zeta+|\bar{\Gamma}| \cos \xi] \cdot(+\sin \theta \cos \phi)\right\} \\
& E_{\phi I+I I}= 2 I(\theta, \phi)\{j[\sin \zeta+|\bar{\Gamma}| \sin \xi] \cdot(+\sin \theta)+ \\
&\left.k\left(\varepsilon_{e f f}\right)^{1 / 2}[\cos \zeta+|\bar{\Gamma}| \cos \xi] \cdot(+\cos \phi)\right\} \\
& \mathbf{H}_{I+I I}=2 I(\theta, \phi)\left\{j [ \operatorname { s i n } \zeta + | \overline { \Gamma } | \operatorname { s i n } \xi ] \cdot \left[\hat{a}_{x}(-\sin \theta \cos \theta \cos \phi)+\right.\right. \\
&\left.\hat{a}_{y}(-\sin \theta \cos \theta \sin \phi)+\hat{a}_{z}\left(+\sin ^{2} \theta\right)\right]+k\left(\varepsilon_{e f f}\right)^{1 / 2}[\cos \zeta+|\bar{\Gamma}| \cos \xi] \\
& {\left[\left(\hat{a}_{x}(-\cos \theta)+\hat{a}_{z}(+\sin \theta \cos \phi)\right]\right\} \cdot \frac{1}{Z_{o}} }
\end{aligned}
$$




\section{REFERENCES}

[1] Steinberg, B.D., 'Principles of Aperture and Array System Design Including Random and Adaptive Arrays,' John Wiley and Sons, New York, 1976.

[2] Hansen, R.C., 'Microwave Scanning Antennas, Vol. 1-Apertures,' Academic Press, New York, 1964.

[3] Jasik, H., 'Antenna Engineering Handbook,' McGraw-Hill Book Co., New York, 1961.

[4] Born, M. and Wolf, E., 'Principles of Optics,' Pergamon Press, New York, 1964.

[5] Hannan, P.W., "Microwave Antenna Derived from Cassegrain Telescope," IRE Trans. on Antenna and Propagation, vol. AP-9, No. 2, pp. 140-153, 1961.

[6] Love, A.W., "Scale Model Development of a High Efficiency Dual Polarized Line Feed for the Arecibo Spherical Reflector," IEEE Trans. on Antennas and Propagation, vol. AP-21, No. 9, pp. 628-639, 1973.

[7] Collin, R.E. and Zucker, F.J, 'Antenna Theory, Pt.1 and Pt.2' McGraw-Hill Book Co., New York, 1969.

[8] Phillips, C.J.E. and Clarricoats, P.J.B., "Optimum Design of a GregorianCorrected Spherical Reflector Antenna," Proc. IEE, 117, No. 4, pp. 718-734, 1970.

[9] Clarricoats, P.J.B. and Poulton, G.T., "High-Efficiency Microwave Reflector Antennas-A Review," Proc. IEEE, Vol. 65, No. 10, pp. 1470-1504, 1977.

[10] Ashmead, J. and Pippardi, A.B., "The Use of Spherical Reflectors as Microwave Scanning Aerials," J. IEE, Vol. 93, Part III-A, pp. 627-632, 1946.

[11] Tingye, L., "A Study of Spherical Reflectors as Wide-Angle Scanning Antennas," IRE Trans. on Antennas and Propagation, vol. AP-7, No. 7, pp. 223-226, 1959.

[12] Spencer, R.C., Sletten, C.J. and Walsh, J.E., "Correction of Spherical Reflector Aberration by a Phased Line Source," Proc. NEC, Vol. 5, pp. 320-333, 1950. 
[13] Rotman, W., "Wide-Angle Scanning with Microwave Double-Layer Pillboxes," IRE Trans. on Antennas and Propagation, vol. AP-6, No. 1, pp. 96-105, 1958.

[14] Schell, A.C., "The Diffraction Theory of Large-Aperture Spherical Reflector Antennas," IEEE Trans. on Antennas and Propagation, vol. AP-11, No. 8, pp. 428-432, 1963.

[15] Holt, F.S. and Bouche, E.L., "A Gregorian Corrector for Spherical Reflectors," IEEE Trans. on Antennas and Propagation, vol. AP-12, No. 1, pp. 44-47, 1964.

[16] Ishimaru, A., Srenivasiah, I. and Wong, V.K., "Double Spherical Cassegrain Reflector Antennas," IEEE Trans. on Antennas and Propagation, vol. AP-21, No. 11, pp. 774-780, 1973.

[17] Chait, H.N., "Wide-Angle Scan Radar Antenna," Electronics, Vol. 26,pp. 128132, Jan. 1953.

[18] Coleman, H.P., "Paraboloidal Reflector off-set fed with a Corrugated Conical Horn," IEEE Trans. on Antennas and Propagation, vol. AP-23, No. 1, pp. 817$819,1979$.

[19] Clarricoats, P.J.B. and Olver, A.D., 'Corrugated Horns for Microwave Antennas,' Peter Peregrinus Ltd., London, UK., 1984.

[20] Kraus, J.D., 'Antennas,' McGraw-Hill Book Co., New York, 1988.

[21] Dyson, J.D., "The Equiangular Spiral Antenna," IRE Trans. on Antennas and Propagation, vol. AP-7, No. 4, pp. 181-187, 1959.

[22] De Vito, G. and Giovanni, B.S., "Further Comments on the Design of LogPeriodic Dipole Antennas," IEEE Trans. on Antennas and Propagation, vol. AP22, No. 9, pp. 714-718, 1974.

[23] Stephenson, D.T. and Finley, S.K., "Use of Log-Periodic Feeds in Corner Reflector," IEEE Trans. on Antennas and Propagation, vol. AP-20, No. 11, pp. 770-772, 1972.

[24] Cumming, W.A., "A Dual-Polarized Line Source for use at S-band," Microwaves J., vol. 6, No. 1, pp. 81-87, 1963. 
[25] Love, A.W. and Gustinic, J.J., "Line Source Feed for a Spherical Reflector," IEEE Trans. on Antennas and Propagation, vol. AP-16, No. 1, pp. 132-134, 1968.

[26] Lalonde, L.M. and Harris, D.E., "A High-Performance Line Source Feed for the AIO Spherical Reflector," IEEE Trans. on Antennas and Propagation, vol. AP-18, No. 1, pp. 41-44, 1970.

[27] Haung, J. and Rahmat-Samii, Y., "Fan Beam Generated by a Linear-Array Fed Parabolic Reflector," IEEE Trans. on Antennas and Propagation, vol. AP-32, No. 7, pp. 1046-1053, 1990.

[28] Minnett, A.C. and Thomas, B.M., "Fields in the Image Space of Symmetrical Focusing Reflectors," Proc. IEE, 115, No. 10, pp. 1419-1430, 1968.

[29] Thomas, B.M., "Theoretical Performance of Prime-Focus Paraboloids using Cylindrical Hybrid-Mode Feeds," Proc. IEE, 118, No. 11, pp. 1539-1549, 1971.

[30] Rudge, A.W. and Adatia, N.A., "Offset-Parabolic-Reflector Antennas:A Review," Proc. IEEE, Vol. 66, No. 12, pp. 1592-1618, 1978.

[31] Rush, W.V.T., "Scattering from a Hyperboloidal Reflector in a Cassegrain Feed System," IEEE Trans. on Antennas and Propagation, vol. AP-11, No. 7, pp. 414$421,1963$.

[32] Galindo, V., "Design of Dual-Reflector Antennas with Arbitrary Phase and Amplitude Distributions," IEEE Trans. on Antennas and Propagation, vol. AP-12, No. 7, pp. 403-408, 1964.

[33] Williams, W.F., "High Efficiency Antenna Reflector," Microwaves J., vol.8, No. 7, pp. 79-82, 1965.

[34] Collins, G.W., "Shaping of Subreflectors in Cassegrain Antennas for Maximum Aperture Efficiency," IEEE Trans. on Antennas and Propagation, vol. AP-21, No. 5, pp. 309-313, 1973.

[35] Pagones, M.J., "Gain factor of an Offset-Fed Paraboloidal Reflector," IEEE Trans. on Antennas and Propagation, vol. AP-16, No. 5, pp. 653-655, 1968. 
[36] Clarricoats, P.J.B. and Salema, P.J.B., "Propagation and Radiation Characteristics of Low-Permittivity Dielectric Cones," Electronics Letters, 7, No. 17, pp. 483$485,1971$.

[37] Shafai, L. and Chugh, R.K., "Dielectric Cones with a Shaped Reflecting Surfaces," Electronics Letters, 12, No. 16, pp. 416-418, 1976.

[38] Chu, T.S. and Turrin, R.H., "Depolarization of Offset Reflector Antennas," IEEE Trans. on Antennas and Propagation, vol. AP-21, No. 5, pp. 339-345, 1973.

[39] Rudge, A.W., Milne, K., Olver, A.D. and Knight, P., 'The Handbook of Antenna Design,' of Aperture and Array Peter Peregrinus, London, U.K., 1986.

[40] Eid, D.A.M. and Shafai, L., "Focal Region Field of Conical Reflectors," IEEE Int. Symp. Digest, Vol. II, pp. 511-514, 1986. June 1986.

[41] Ludwig, A.C., "A new geometry for unfurlable antennas," Microwaves, vol. 9, No. 11, pp. 41-42, 1970.

[42] Ludwig, A.C., "Conical reflector antennas," IEEE Trans. on Antennas and Propagation, vol. AP-20, No. 3, pp. 146-152, 1972.

[43] Olver, R.E., "Large Spacecraft Antennas: New Geometric Configuration Design Concepts," Jet Propulsion Lab. Quarterly Technical Review, vol. 1, No. 1, pp. 78-80, 1971.

[44] Love, A.W., "Some Highlights in Reflector Antenna Development," Radio Science, vol. 11, No. 8, pp. 671-684, 1976.

[45] Eid, D.A.M. and Shafai, L., "Synthesis of Line Feeds for Conical Reflectors," Antenna Technology and Applied EM (ANTEM) Proc. (86), Winnipeg, Canada, August 1986.

[46] Eid, D.A.M. and Shafai, L., "The Field Distribution in the Focal Plane of Conical Reflectors," First International Conference on Engineering Mathematics and Physics Proc., Cairo, Egypt, February 1991.

[47] Ghobrial, S.I, "Co-Polar and Cross-Polar Diffraction Images in the Focal Plane of Paraboloidal Reflector. A comparison between Linear and Circular Polarization," 
IEEE Trans. on Antennas and Propagation, vol. AP-24, No. 9, pp. 418-424, 1976.

[48] Silver, S., 'Microwave Antenna Theory and Design,' McGraw-Hill Book Co., New York, 1984.

[49] James, M.I., Smith, G.M. and Wolfor, J.C., 'Applied Numerical Methods for Digital Computation with Fortran and CSMP,' Harper \& Row Publishers, New York, 1977.

[50] Rydahl, O., 'Scattering from Small Bodies of Revolution,' M.Sc. Thesis, Electromagnetic Institute, Tech. University of Denmark, Lyngby, Sept. 1974.

[51] Jordan, E.C., and Balmain, K., 'Electromagnetic Waves and Radiating Systems,' Prentice-Hall Inc., Englewood Cliffs., N.J., 1970.

[52] Hamid, M.A.K., Boerner, W.M., Shafai, L., Towaij, S.J., Alsip, W.P., and Wilson, G.J., "Radiation Characteristics of Bent Wire Antennas," IEEE Trans. Electromagn. Compat., vol. EMC-12, No. 3, pp. 106-111, 1970.

[53] Rush, W.V.T., and Potter, P.D., 'Analysis of Reflector Antennas,' McGraw-Hill Book Co., New York, 1977.

[54] Eid, D.A.M. and Shafai, L., "Radiation from Conical Reflectors with a Dipole Array Feed," Antenna Technology and Applied EM (ANTEM) Proc. (88), Winnipeg, Canada, August 1988.

[55] Eid, D.A.M. and Shafai, L., "The Performance of Conical Reflectors with a Dipole Array Feed," Antenna Technology and Applied EM (ANTEM) Proc. (90), Winnipeg, Canada, August 1990.

[56] Ludwig, A.C., "The Definition of Cross-Polarization," IEEE Trans. on Antennas and Propagation, vol. AP-12, No. 1, pp. 116-119, 1973.

[57] Eid, D.A.M. and Shafai, L., "Dipole Array Feeds for Conical Reflectors," First International Conference on Engineering Mathematics and Physics Proc., Cairo, Egypt, February 1991. 
[58] Eid, D.A.M. and Shafai, L., "Synthesis of Line Source Feeds for Conical Reflectors and their Performance using Dipole Arrays," Cand. J. Phys., vol. 69, No. 6, pp. 702-711, 1991.

[59] James, J.R., Hall, P.S. and Wood, C., 'Microstrip Antenna Theory and Design,' Peter Peregrinus Ltd., Stevenage, U.K, and New York, 1977.

[60] James, J.R. and Wilson, G.J., "Microstrip Antennas and Arrays. Pt. 1- Fundamental Action and Limitations," IEE J Microwaves, Optics and Acoustics, vol. MOA-1, No. 5, pp. 165-174, 1977.

[61] James, J.R. and Hall, P.S., "Microstrip Antennas and Arrays. Pt. 2- New ArrayDesign Technique," IEE J Microwaves, Optics and Acoustics, vol. MOA-1, No. 5, pp. 175-181, 1977.

[62] Wood, C., "Curved Microstrip Lines as Compact Wideband Circularly Polarized Antennas," IEE J Microwaves, Optics and Acoustics, vol. MOA-3, No. 1, pp. 5$13,1983$.

[63] Hall, P.S., "Microstrip Linear Array with Polarization Control," IEE J Microwaves, Optics and Acoustics, vol. MOA-130, No. 3, pp. 215-224, 1983.

[64] Shafai, L. and Sebak, A.A., "Radiation Characteristics and Polarization of Undulated Microstrip Line Antennas," IEE J Microwaves, Optics and Acoustics, vol. MOA-132, No. 7, pp. 433-439, 1985.

[65] Collin, R.E., and Zucker, F.G., 'Antenna Theory part 1,' McGraw-Hill Book Co., New York, 1969.

[66] Bahl, I.J. and Bhartia, P., 'Microstrip Antennas,' Artech-House Inc., 1980.

[67] James, J.R. and Labrooke, Ph., "Surface Wave Phenomena associated with OpenCircuited Strip Line Terminations," Electron Lett.,No. 9, pp. 571-572, 1973.

[68] GUPTA, K.C., Garge, R., and Bahl I.J., 'Microstrip Lines and Slot Lines,' Artech-House Inc., 1979.

[69] James, J.R. and Handerson, A., "High-Frequency Behavior of Microstrip OpenCircuit Terminations," IEE J Microwaves, Optics and Acoustics, vol. MOA-3, No. 
5, pp. 205-218, 1979.

[70] Eid, D.A.M. and Shafai, L., "The Performance of Conical Reflectors with a Microstrip Comb Line Array Feed," Antenna Technology and Applied EM (ANTEM) Proc. (92), Winnipeg, Canada, August 1992. 